\title{
RIQUEZA FITOPLANCTÓNICA DE LA BAHÍA DE ACAPULCO Y ZONA COSTERA ALEDAÑA, GUERRERO, MÉXICO
}

\author{
María Esther Meave-del Castillo ${ }^{1,4}$, María Eugenia Zamudio-Resendiz ${ }^{2}$ y \\ Manuel CAstillo-Rivera ${ }^{3}$
}

${ }^{1}$ Universidad Autónoma Metropolitana-Iztapalapa, Departamento de Hidrobiología, Laboratorio de Fitoplancton Marino y Salobre, Av. San Rafael Atlixco186, Colonia Vicentina, Del. Iztapalapa, 09340 México, D.F., México.

${ }^{2}$ Estudiante de Doctorado en Ciencias Biológicas y de la Salud. Universidad Autónoma Metropolitana, Unidad Iztapalapa.

${ }^{3}$ Universidad Autónoma Metropolitana-Iztapalapa. Departamento de Biología, Laboratorio de Peces, Avenida San Rafael Atlixco 186, Colonia Vicentina, Del. Iztapalapa, 09340 México, D.F., México. ${ }^{4}$ Autor para la correspondencia: mem@xanum.uam.mx

\section{RESUMEN}

Se presentan los resultados del inventario de la ficoflora planctónica de la bahía de Acapulco y zona marina aledaña, reconocida a través de una década de estudios, incluyendo uno intensivo con muestreos bimestrales usando red de fitoplancton, botella y observación de muestras vivas, de febrero/2010 a febrero/2011. Se reconocieron 641 taxa, pertenecientes a ocho divisiones algales. El grupo más diverso fue Dinophyta, con 347 taxa, seguido de Bacillariophyta con 274 taxa. Se incluyen fotografías de 131 taxa, 38 correspondientes a nuevos registros para el Pacífico Mexicano, 34 no identificadas al nivel de especie y 59 que tienen algún interés taxonómico-ecológico. El análisis de las curvas de acumulación de especies permite afirmar que la riqueza fitoplanctónica de la Bahía de Acapulco conocida hasta el momento es ya representativa de la ficoflora potencial del sitio; sin embargo, faltan aún estudios más detallados sobre fitoflagelados y picoplancton. Se hace la caracterización biológica de la comunidad fitoplanctónica en términos de: nivel de organización, tipo de nutrición, forma de vida, potencialidad de nocividad o toxicidad, origen (dulceacuícola, salobre o marina), afinidad (nerítica u oceánica) y distribución (cosmopolita, fría-templada, subtropical o tropical). Se reconocen las especies típicas de la bahía y aquellas que han producido florecimientos. La riqueza ficoflorística encontrada en Acapulco puede reflejar una condición trófica variable a lo largo del año, con limitación de nutrientes en ciertos 
momentos, hecho que se refuerza por las relaciones simbióticas encontradas entre feosomas de cianofitas con dinoflagelados y diatomeas; sin embargo, la presencia de otras especies indicadoras de contaminación, tales como Euglena pascheri, Phaeocystis sp. y el consorcio Leptocylindrus mediterraneus (diatomea)-Solenicola setigera (protozoario), muestran que en otros momentos las concentraciones de nutrientes y materia orgánica pueden ser altas. La forma típica de "anfiteatro" que presenta la bahía, propicia que en la época de lluvias reciba gran cantidad de desechos y aguas negras procedentes de los cerros circundantes sumamente poblados. Sin embargo, la bahía de Acapulco parece tener una alta resilencia, probablemente como resultado de un papel conjugado entre las corrientes marinas (que producen un efecto de lavado de la contaminación antropógena) y la depuración producida por la diversidad funcional de la comunidad fitoplanctónica.

Palabras clave: Bacillariophyta, biodiversidad, Dinophyta, fitoplancton, nuevos registros, Pacífico Oriental Tropical.

\begin{abstract}
We present the inventory of the planktonic algal flora of the Bahía de Acapulco and surrounding marine area, studied through a decade, including an intensive bimonthly sampling study using plankton net, bottle method and the observation of live samples, from February 2010 to February 2011. It recognized 641 taxa, corresponding to eight algal divisions. The most diverse groups were Dinophyta and Bacillariophyta with 347 and 274 taxa, respectively. Photographs of 131 taxa are presented, corresponding to 38 new records for the Mexican Pacific, 34 taxa were not identified at species level, and 59 taxa represent some taxonomic or ecological interest. Species accumulation curves allow to recognize that the species richness observed in the present study represents a good approximation of the planktonic algal flora from the Bahía de Acapulco. However, more detailed studies over phytoflagellates and picoplankton are necessary. A biological characterization of the phytoplankton community was made according to organization level, type of nutrition, life form, potential harmfulness or toxicity, origin (freshwater, brackish or marine), affinity (neritic or oceanic) and distribution (cosmopolitan, cold-temperate or subtropical-tropical). It recognized the common species of the bay and those that have produced blooms. The high species richness of planktonic algal flora observed in Acapulco may reflect a variable trophic status throughout the year, with nutrient limitation at certain times, condition which agrees with the symbiotic relationships found among phaeosomes (cyanophytes), dinoflagellates and diatoms. However, some species indicative of pollution such as Euglena pascheri,
\end{abstract}


Phaeocystis sp. and the presence of the consortium Leptocilindrus mediterraneus (diatom)Solenicola setigera (protozoan), show that some times the concentrations of nutrients and organic matter can be high. The typical form of "amphitheater" that characterizes the bay causes that, during the rainy season, the system receives an input of large amounts of waste and sewage from the surrounding densely populated hills. However, Acapulco Bay seems to have a high resilience, probably as a result of a conjugated role between ocean currents (which produce a washing effect of the anthropogen pollution) and the purification produced by the functional diversity of the phytoplankton community.

Key words: Bacillariophyta, biodiversity, Dinophyta, new records, phytoplankton, tropical Eastern Pacific.

\section{INTRODUCCIÓN}

El fitoplancton marino, principal componente fotosintético de los ecosistemas pelágicos, es una comunidad diversa, constituida por alrededor de 5,000 especies de microalgas, distribuidas en aproximadamente 19 clases de 7 divisiones (Tett \& Barton, 1995; Lee, 1999; Hernández- Becerril, 2003); entre las que destacan por su riqueza y abundancia Bacillariophyta (diatomeas) y Dinophyta (dinoflagelados). Esta es una comunidad compleja, toda vez que aunque en su mayoría son organismos fotosintéticos y por tanto autótrofos, varias especies son mixótrofas, o incluso totalmente heterótrofas, jugando un papel importante no solo como productores primarios, sino también como consumidores primarios y reciclando en corto tiempo nutrientes en la columna de agua (Gaines \& Elbrächter, 1987; Hansen, 1991). Asimismo, principalmente en zonas oligotróficas, se desarrollan interesantes relaciones simbióticas de todo tipo: comensalismo, mutualismo y parasitismo, que permiten a los organismos explotar los recursos del ambiente y sobrevivir bajo condiciones de limitación (Margalef, 1978; Taylor, 1982). Como parte de esta complejidad, en ambientes turbulentos es común encontrar dentro de la comunidad fitoplanctónica, organismos denominados ticoplancton, correspondientes a algas de hábitos principalmente bentónicos (microfitobentos) que son desprendidas del sustrato y acarreadas por las corrientes a la zona pelágica, pero que al no presentar las adaptaciones propias de las especies planctónicas para mantenerse suspendidas, se sedimentan rápidamente al disminuir la energía externa del sistema (Porter, 2008).

De manera general, los cambios en la composición de las comunidades fitoplactónicas responden a condiciones ambientales tales como: luz, temperatura, nu- 
trientes, turbulencia y patrones de circulación del agua (Reynolds, 1997; Alves-deSouza et al., 2008). Además como respuesta a diversos factores, entre los que se encuentran el incremento en las concentraciones de nutrientes, se producen proliferaciones exacerbadas de ciertas especies, causando los eventos denominados como florecimientos algales nocivos (FAN), que tienen consecuencias negativas sobre la biota acuática, debido a que provocan anoxia en la columna de agua, causan daños mecánicos sobre las branquias de los peces, o producen toxinas que envenenan o enferman a otros organismos (Reguera, 2002). En el fitoplancton marino existen unas 300 especies nocivas, de las cuales unas 40 (principalmente dinoflagelados) son tóxicas (Hallegraeff, 2004).

La región del Pacífico Mexicano (PM) presenta una riqueza fitoplanctónica importante, habiéndose registrado como mínimo 875 taxa de diatomeas (Hernández-Becerril \& Díaz-Almeyda, 2006; Hernández-Becerril et al., 2010; Meave, 1999, 2002, 2006, 2009; Meave et al., 2001, 2003b, 2008; Moreno et al., 1996; MorenoGutiérrez, 2008; Sterrenburg et al., 2003) y 620 dinoflagelados, principalmente en la región costera (Gárate-Lizárraga, 2008; Gárate-Lizárraga \& Verdugo-Díaz, 2007; Gárate-Lizárraga et al., 2009; Hernández-Becerril et al., 2003, 2008; Hernández-Becerril \& Bravo-Sierra, 2004a,b; Meave et al., 2003b; Okolodkov \& Gárate-Lizárraga, 2006; Licea et al., 1995). Debido a su amplitud geográfica (de los $33^{\circ} \mathrm{N}$ a los $\left.14^{\circ} \mathrm{N}\right)$, el PM puede dividirse en varias subregiones de acuerdo con sus características climáticas en: a) Pacífico subtropical-templado (Costa Occidental de Baja California), b) Golfo de California, c) Zona de Transición (Bahía de Banderas y zona aledaña), d) Pacífico tropical mexicano (PTM), y e) Golfo de Tehuantepec (Pacheco-Sandoval, 1991; Hendricks, 1993; Meave et al., 2003a). En la porción subtropical-templada, en general, las diatomeas constituyen la comunidad dominante en el fitoplancton, mientras que en la tropical los dinoflagelados llegan a ser más relevantes, o bien su abundancia se intercala en periodos de tiempo con las diatomeas. En particular la parte central del PTM, correspondiente a las costas de los estados de Guerrero y Michoacán, ha mostrado tener una alta riqueza fitoplanctónica (Meave, 2006); seguramente debido a la influencia de corrientes antagónicas (Corriente de California y Corriente Costera de Costa Rica), que producen condiciones fisicoquímicas contrastantes en distintas épocas del año (Transviña, 2002), creando ambientes diversos que posibilitan la expresión de un alto número de especies.

A pesar de que la bahía de Acapulco es uno de los sitos turísticos más concurridos dentro de la porción tropical del PM, y en consecuencia con alto impacto ambiental, son pocas las publicaciones de su flora planctónica. Existe el antecedente de la contribución de Osorio-Tafall (1942), que aunque relevante, es un trabajo an- 
tiguo realizado en la región oceánica de la costa de Acapulco y no propiamente en la bahía, cuya flora planctónica actual podría estar bastante modificada. Asimismo existen dos artículos recientes, uno correspondiente a un florecimiento puntual de una especie tóxica (Gárate-Lizárraga et al., 2012) y el otro, un antecedente importante para el presente estudio, ya que corresponde a un reporte de la composición y abundancia de la comunidad fitoplanctónica de Acapulco, en un intervalo de ocho meses consecutivos realizado en el año 2009 (Rojas-Herrera et al., 2012), en el cual se registran 87 especies de microalgas en la columna de agua; seis de ellas reconocidas como dominantes (cuatro diatomeas y dos dinoflagelados). Por el contrario se han realizado estudios faunísticos de varios grupos: zooplancton (Balleza-García \& Nestor-Cervantes, 1992; Martínez-Orozco, 2004; Ramírez-Rosas, 2004), poliquetos (Salcedo-Oropeza, 2011), moluscos (García-López, 1994; Barba-Marino, 2009), esponjas (Aguilar-Camacho, 2010), equinodermos (Lucero-Blanco \& Herrera-Hernández, 2007), peces (Rendón-Gómez, 1995), reptiles (Nava-Cuevas, 2008), y aves (Cárdenas-Santiago \& Jiménez-Escobar, 2001).

El objetivo del presente estudio es mostrar la riqueza fitoplanctónica de la Bahía de Acapulco, a través de los estudios realizados en una década, por personal del Laboratorio de Fitoplancton Marino y Salobre del Departamento de Hidrobiología de la Universidad Autónoma Metropolitana-Unidad Iztapalapa, analizando el esfuerzo de muestreo, reconociendo las especies típicas de la bahía, evaluando además características biológicas y biogeográficas de las especies que conforman las comunidades fitoplanctónicas, para tener una idea de su complejidad, tales como: afinidad biogeográfica, tipo de nutrición (incluyendo aspectos de simbiosis mutualistas o parasitismo), hábito (o forma de vida) y nivel de organización. Además, dado que los fenómenos de Florecimientos Algales Nocivos (FAN) son frecuentes en la bahía de Acapulco (com. pers. miembros del Laboratorio Estatal de Salud Pública del Estado de Guerrero), en este trabajo se hace énfasis también en el reconocimiento de las microalgas tóxicas y/o nocivas, presentes y recurrentes en la bahía; así como en los taxa que por su abundancia se han exhibido como florecimientos.

Es importante señalar que la presente corresponde a la primera publicación de una serie planeada, con las cuales se analizará no solo la presencia de las especies planctónicas, sino también su distribución, por lo que en esta contribución nos hemos concentrado en registrar el listado y cualidades de las algas encontradas en distintos sitios y momentos en la bahía de Acapulco, a lo que hemos llamado riqueza, de acuerdo con Magurran (2004), para que sobre esta base, en una publicación subsiguiente, abordar aspectos de la estructura de las comunidades. Sin embargo, a pesar de que en esta publicación no se hará un análisis exhaustivo de los datos am- 
bientales, en los resultados mostramos también los valores promedio mensuales de varios parámetros fisicoquímicos, registrados en más de 40 sitios, desde febrero de 2010 hasta febrero de 2011, con datos adicionales de octubre de 2009.

\section{MATERIALES Y MÉTODOS}

Área de estudio

La bahía de Acapulco se localiza en la plataforma del estado de Guerrero (99 $50^{\prime} 52^{\prime \prime}-99^{\circ} 56^{\prime} \mathrm{N}$ y $16^{\circ} 47^{\prime}-16^{\circ} 51^{\prime} 40^{\prime \prime}$ O; Fig. 1); tiene una forma semicircular, una longitud aproximada de $7 \mathrm{~km}$ y una anchura promedio de $10 \mathrm{~km}$. Sus profundidades oscilan entre los 10 y $30 \mathrm{~m}$, pero dada su cercanía con la Trinchera Mesoamericana (Flamand-Swaner, 1991), apenas en su entrada ya existen profundidades de $50 \mathrm{~m}$ y $20 \mathrm{~km}$ mar adentro, incluso los $400 \mathrm{~m}$. Toda la bahía tiene sedimentos arenosos, pero hacia la boca se encuentran sedimentos más finos: arenas lodosas y lodos arenosos (Emery, 1967; Kulm et al., 1975), porque los sedimentos finos se mantienen suspendidos, depositándose hasta llegar a mar abierto. El relieve costero tiene forma de anfiteatro (Ramírez, 1987), creado por un macizo de montañas que rodean a la bahía con una altitud promedio de $500 \mathrm{~m}$, con un bloque montañoso al norte, de $920 \mathrm{~m}$, que se extiende en dirección NO-SE, y otro al sur, de $460 \mathrm{~m}$ de altitud en el Cerro del Vigía. De estas montañas, durante la época de lluvias surgen una serie de arroyos temporales que drenan a la bahía (Fig. 1), y que son el principal medio de transporte de materia orgánica y basura, desde la zona urbana hasta la costera, contribuyendo con ello a su contaminación (Mayo-Vera, 2004). De hecho, uno de los arroyos de aguas negras más importantes, denominado El Camarón, descarga entre las playas de Tamarindos y Hornitos, justo en la parte central de la bahía, frente al Morro del Obispo.

El clima es de tipo Aw (tropical lluvioso con lluvias en verano; Tamayo, 1970), con temperaturas mayores de $18{ }^{\circ} \mathrm{C}$ todo el año, precipitaciones promedio mayores de $100 \mathrm{~mm}$ entre junio y octubre (temporada de lluvias) y menores de $4 \mathrm{~mm}$ entre febrero y abril (temporada de secas; Mayo-Vera, 2004). De mayo a noviembre la región es afectada por depresiones tropicales, que pueden convertirse en tormentas tropicales y en ocasiones formar huracanes o ciclones (Tamayo, 1970).

Aunque existen pocos estudios sobre las corrientes de la bahía, dos de ellos, el de la Secretaría de Marina (Anónimo, 1979) y el de Dionni y Romo (1984, en Ramírez-Rosas, 2004), señalan que entre la isla La Roqueta y la costa occidental de la bahía, se genera una corriente con velocidad aproximada de $30 \mathrm{~cm} / \mathrm{seg}$ (Anónimo, 


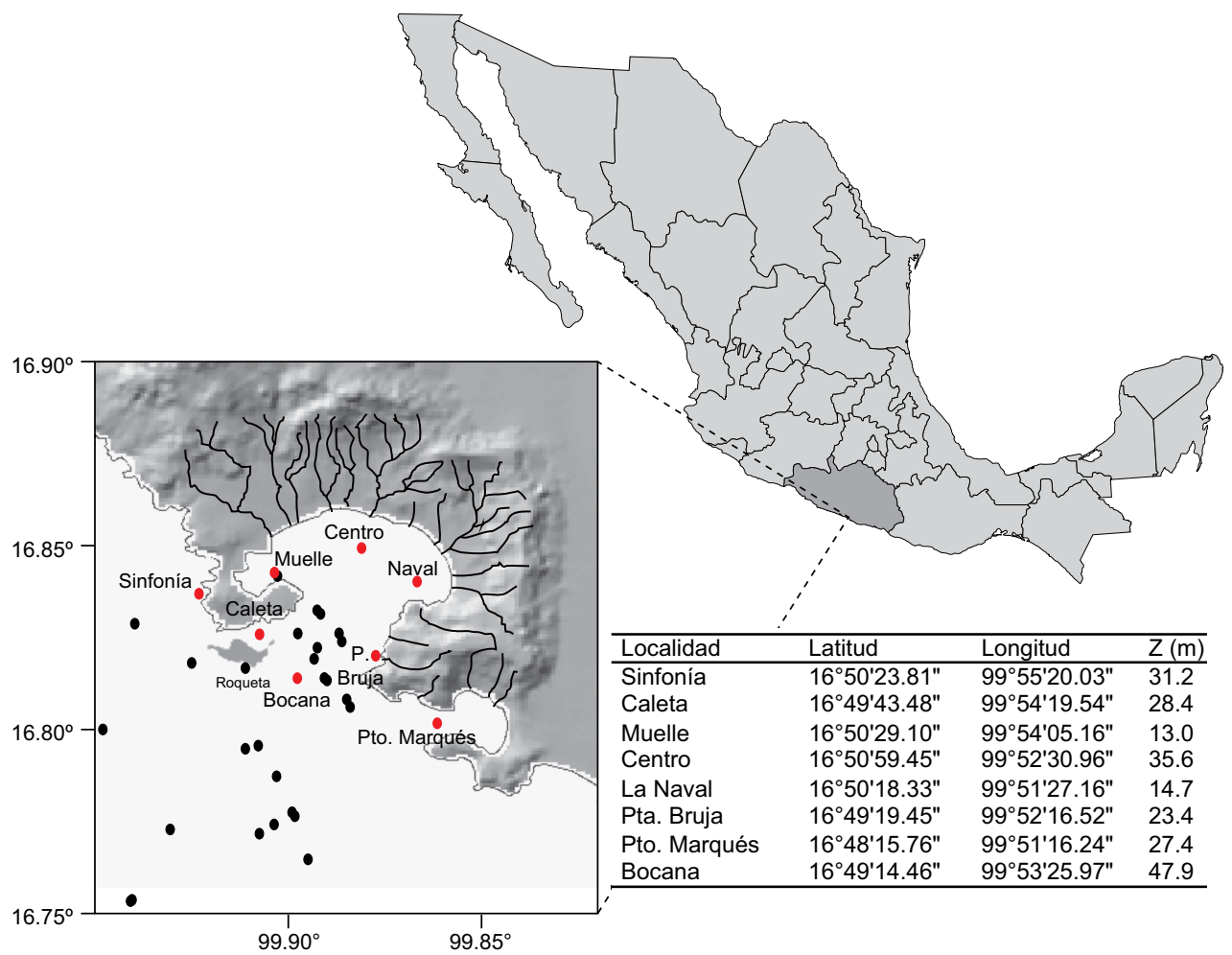

Fig. 1. Ubicación de área de estudio, con topografía costera, hidrografía y sitios de colecta en la Bahía de Acapulco y zona marina aledaña. En rojo los ocho sitios donde se colectó bimestralmente durante el ciclo de febrero de 2010 a febrero de 2011, indicando en la tabla adjunta las coordenadas geográficas y la profundidad total de estos sitios. Todos los sitios marcados en negro y ubicados por fuera de la Bocana, se consideran localidades costeras. El mapa se construyó a partir de la línea de costa obtenida del Shoreline/coastline Resources del National Geophysical Data Center (NOAA), al cual se le agregaron los datos de topografía e hidrografía de cartas obtenidas de Instituto Nacional de Geografía y Estadística (Anónimo, 2005). Se ubicaron los 41 sitios muestreados en la bahía de Acapulco y zona marina aledaña desde el año 2000, con ayuda del software SigmaPlot10 (Systat Software Inc., 2006).

1979) que produce un hundimiento de agua en las proximidades del Bajo La Hierbabuena, lo que junto con los vientos que soplan del oeste, influencia la existencia de corrientes con dirección oriental en el interior de la misma, de manera que el agua la recorre y vuelve a salir a mar abierto a la altura de Punta Bruja (Fig. 1).

Con respecto a las condiciones ambientales de la columna de agua de Acapulco, Domínguez-Parra (1979) en un estudio realizado entre octubre de 1978 a 
marzo de 1979, mostró que eran muy variables, con temperaturas promedio de 28.5 a $30.5^{\circ} \mathrm{C}$, transparencia de 3.8 a $11.1 \mathrm{~m}$, salinidad de 31.97 a 34.98 , concentraciones de oxígeno de 3.73 a $4.45 \mathrm{mg} \mathrm{L}^{-1}$, de amonio de 0.73 a $1.68 \mathrm{mg} \mathrm{L}^{-1}$, de nitratos de 0.43 a $0.47 \mathrm{mg} \mathrm{L}^{-1} \mathrm{y}$ de fosfatos de 0.034 a $0.332 \mathrm{mg} \mathrm{L}^{-1}$.

Muestreo de fitoplancton

La información sobre la riqueza fitoplanctónica de la Bahía de Acapulco se obtuvo a través del análisis de 484 muestras, de las cuales 88 son muestras fijadas correspondieron a las recolectadas con red, 364 a las fijadas obtenidas con botella Van Dorn y 32 a las observadas en vivo (Cuadro 1). Las muestras fueron tomadas dentro de la bahía y zona costera aledaña, cubriendo diferentes épocas climáticas y sitios georreferenciados con GPS, entre mayo de 2000 y octubre de 2009 (Cuadro 1, Fig.

Cuadro 1. Número y fecha de colecta de muestras de fitoplancton revisadas (Núm. M.) por tipo de muestreo (botella, red o viva).

\begin{tabular}{|c|c|c|c|c|c|}
\hline \multicolumn{2}{|c|}{ Botella } & \multicolumn{2}{|c|}{ Red* } & \multicolumn{2}{|c|}{ Viva } \\
\hline Fecha & Núm. M. & Fecha & Núm. M. & Fecha & Núm. M. \\
\hline Nov./2007 & 1 & Mayo/2000 & 8 & Oct./09 & 4 \\
\hline Mayo/2008 & 10 & Sept./2000 & 5 & Marzo/2010 & 4 \\
\hline Junio/2008 & 27 & Oct.20/00 & 5 & Mayo/2010 & 4 \\
\hline Sept./2008 & 11 & Nov./2002 & 12 & Julio/2010 & 8 \\
\hline Oct./2009 & 37 & Mayo/2003 & 5 & Sept./2010 & 4 \\
\hline Marzo/2010 & 38 & Junio/2003 & 2 & Nov./2010 & 4 \\
\hline Mayo/2010 & 41 & Nov./2003 & 3 & Enero/2011 & 4 \\
\hline Julio/2010 & 80 & Junio/2004 & 6 & & \\
\hline Sept./2010 & 40 & Nov./2006 & 4 & & \\
\hline Nov./2010 & 38 & Marzo/2007 & 2 & & \\
\hline \multirow[t]{6}{*}{ Enero/2011 } & 41 & Feb./2008 & 7 & & \\
\hline & & Feb./2009 & 1 & & \\
\hline & & Oct./2009 & 9 & & \\
\hline & & Мayo/2010 & 10 & & \\
\hline & & Junio/2010 & 8 & & \\
\hline & & Julio/2010 & 1 & & \\
\hline Total & 364 & & 88 & & 32 \\
\hline
\end{tabular}

*Incluye arrastre horizontal y vertical, realizados en el mismo sitio. 
1), y a partir del mes de febrero de 2010 hasta febrero de 2011, se realizó un muestreo intensivo, sistemático y continuado, con recolectas mensuales en 5 sitios en el interior de la bahía (Muelle, Centro, La Naval, Punta Bruja y Bocana) y 3 en la zona costera aledaña (Sinfonía, Caleta y Puerto Marqués; Fig. 1); toda la información fue vertida en la base de datos Biotica (CONABIO) para facilitar el manejo de la información.

Se usó una embarcación de motor, para la recolección del material con redes de fitoplancton de $54 \mu \mathrm{m}$ y $20 \mu \mathrm{m}$ de abertura de poro, mediante arrastres horizontales y verticales, fijando el material in situ con formalina neutralizada (hasta alcanzar una concentración final de 4\%). También se tomaron muestras con botella Van Dorn (a 1, 3, 5, 10, 20, 30 y 50 m, o fondo según la profundidad del sitio), las cuales fueron preservadas con una solución de lugol ácido (Throndsen, 1978). Todas las muestras fijadas fueron depositadas en la colección de Fitoplancton Marino (FpM) de la Universidad Autónoma Metropolitana-Unidad Iztapalapa.

Con la idea de registrar dinoflagelados desnudos y fitoflagelados (organismos frágiles que se afectan severamente o explotan con los fijadores), durante los muestreos mensuales del año 2010-2011, se obtuvieron también materiales con botella Van Dorn a 1, 3 y $5 \mathrm{~m}$ de profundidad, con las cuales se llenó a tope un garrafón de $10 \mathrm{~L}$, que fue protegido de la luz y cambios de temperatura hasta su transporte a tierra, donde el material se concentró a través de un sistema de filtración inversa (Dodson \& Thomas, 1978), observándose inmediatamente bajo el microscopio óptico. De los organismos vivos se tomaron videos cuando su movimiento era activo, y/o fotografías. En el caso de los videos, las imágenes se separaron como fotogramas.

\section{Parámetros físico-químicos}

Desde febrero de 2010 hasta febrero de 2011 (incluyendo también datos de octubre de 2009), se evaluaron mensualmente los parámetros fisicoquímicos: temperatura del agua, salinidad y oxígeno disuelto con ayuda de sondas multiparamétricas (YSI-556 MPS, YSI-550A y Thermo-Orion StarTM), las cuales fueron previamente calibradas según las recomendaciones del fabricante. Para la evaluación de los nutrientes: nitratos+nitritos $\left(\mathrm{NO}_{3+} \mathrm{NO}_{2}\right)$, amonio $\left(\mathrm{NH}_{4}\right)$, ortofosfatos $\left(\mathrm{PO}_{4}\right)$, silicatos $\left(\mathrm{SiO}_{2}\right)$, así como la biomasa fitoplanctónica (Cl- $\left.a\right)$, las muestras de agua fueron filtradas in situ y congeladas hasta su análisis en el laboratorio (con filtros GF/F de $0.7 \mu \mathrm{m}$ de abertura de poro). Para la determinación de nutrientes se emplearon las siguientes técnicas: nitratos más nitritos, método de reducción por columnas Cd-Cu (Strickland \& Parsons, 1972); para el amonio el de azul de indofenol (Solórzano, 1969); para los ortofosfatos el del ácido ascórbico-molibdato (Murphy \& Riley, 1962); y los silicatos se determinaron de acuerdo con el método del ácido p- 
silicomolíbdico (Schwartz, 1942). Para evaluar la biomasa (Cl- $a$ ) se utilizó el método espectrofotométrico (Parsons et al., 1984).

A partir de los datos diarios de la estación meteorológica ubicada en Acapulco (Anónimo, 2010b), se obtuvieron los promedios mensuales de precipitación pluvial del periodo de 1973 a 2009 y se compararon con los del año 2010, con el fin obtener las variaciones del año de estudio (2010), con respecto al promedio de los 36 años anteriores.

Trabajo de laboratorio

Los organismos fueron observados con un microscopio Leica-Diastar DMLB (con iluminación de campo claro, contraste de fases y fluorescencia). Para facilitar la identificación de las especies, las frústulas de las diatomeas se oxidaron siguiendo el método de Simonsen (1974), realizando laminillas permanentes con resina sintética con un alto índice de refracción (Hasle, 1978). En el caso de los dinoflagelados, para facilitar la observación de las placas de su teca celulósica, se usó el colorante Tripan blue e iluminación de campo claro o bien el fluorocromo Calcofluor White y fluorescencia (Fritz \& Triemer, 1985). Algunas muestras fueron seleccionadas para su observación al microscopio electrónico de barrido (JEOL JSM-5900LV, con 10-15 kv de voltaje de aceleración).

Los taxa fueron identificados hasta el nivel de género, siguiendo los esquemas de Round et al. (1990) para Bacillariophyta; Fensome et al. (1993) para Dinoflagellata, sin embargo, en este estudio se clasifican a los dinoflagelados como algas de la división Dinophyta, según el sistema de clasificación de Hoek et al. (1995); Heimdal (1997) y Hoek et al. (1995) para Heterocontophyta; Throndsen (1997) y Whittaker (1959) para Prymnesiophyta y Euglenophyta respectivamente; Bourrelly (1972) para Chlorophyta; y Komárek \& Anagnostidis (2005) para Cyanophyta.

Con la idea de caracterizar a la comunidad microalgal, se registró el nivel de organización de cada taxon, reconociéndose cuatro categorías: a) unicelular móvil o flagelada, b) unicelular no flagelada, c) colonia-cenobio, d) filamento-cadena.

A través de la consulta de las obras de Gaines \& Elbrächter (1987), Prézelin (1987), Balech (1988), Bérard-Therriault et al. (1999), Hasle y Syvertsen (1997), Kofoid y Swezy (1921), Steidinger y Tangen (1997), y de las propias observaciones, se registró el tipo de nutrición de cada taxon, reconociéndose las siguientes tres categorías: a) fotosintéticas, b) mixotróficas, y c) heterótrofas, incluyendo a las osmótrofas, las fagótrofas y a las parásitas.

Se registró el hábito o forma de vida de cada taxon, definiendo si eran planctónicos o ticoplanctónicos (Porter, 2008), usando principalmente las obras de Meave 
et al. (2003a), Krayesky et al. (2009) y Steidinger y Tangen (1997). Asimismo se registró la distribución mundial de cada una de las especies, con base en la información de Bérard-Therriault et al. (1999), Hasle y Syvertsen (1997), Kofoid y Swezy (1921), Licea et al. (1995) y Moreno et al. (1996), considerando cuatro categorías: Tropical (Tr), Subtropical (Sbt), Templada (Tm) y Fría (Fr). Se anotó además su ubicación ecológica: dulceacuícola, salobre o marina y su afinidad nerítica (especies costeras) u oceánica (especies de mar abierto).

Con base en la premisa de que la mayoría de las especies nocivas, aun teniendo la capacidad de generar toxinas pueden producirlas o no, dependiendo de ciertas condiciones ambientales y de su abundancia, se consideró también el potencial de toxicidad de cada uno de los taxa, con base en la revisión de literatura (Steidinger \&Tangen, 1997; Cronberg et al., 2004; Fryxell \& Hasle, 2004; Hallegraeff \& Hara, 2004; Moestrup \& Thompsen, 2004; Taylor et al., 2004).

En el caso de las muestras de botella, además de hacer la identificación de las especies, se cuantificó su abundancia por el método de Utermöhl (Edler \& Elbrächter, 2010), usando un microscopio invertido Motic y cubetas de sedimentación de 25, 50 o $100 \mathrm{ml}$, dependiendo de la concentración de fitoplancton en las capturas. Se determinó la abundancia relativa de la composición de taxa por muestra, tanto en las de red como en las de botella. En el caso de las primeras, los conteos se hicieron sobre preparaciones en fresco, contando células a través de transectos hasta llegar a 400; y en las de las segundas, estos se hicieron sobre las cámaras de sedimentación, mediante el método de transectos a 40x o 60x, hasta llegar a 400 células. Los valores obtenidos fueron convertidos a porcentaje para obtener la abundancia relativa, estableciéndose cuatro categorías: raras $(<10 \%)$, escasas $(10-<30 \%)$, comunes $(30-<80 \%)$ y abundantes $(>80 \%)$; los taxa contenidos en esta última fueron considerados como los formadores de florecimientos. La frecuencia de aparición de cada uno de los taxa se obtuvo sumando el número de muestras donde cada uno de ellos estuvo presente, y dividiendo tal valor entre el número total de muestras revisadas $(\mathrm{n}=484)$. Las especies típicas del fitoplancton de la bahía, son aquellas que resultaron con valores de frecuencia mayores de $50 \%$.

Además se evaluó si el esfuerzo de muestreo realizado era representativo de la riqueza total de especies presente en la bahía, para lo cual se desarrollaron curvas de acumulación de especies (Palmer, 1990; Magurran, 2004) con el programa informático PRIMER versión 6.1.4, utilizando la constante de riqueza máxima $\left(\mathrm{S}_{\max }\right)$ del estimador de Michaelis-Menten, para generar una asíntota con 100 permutaciones, con el fin de mejorar su estimación (Clarke \& Gorley, 2006). Para las muestras de 
botella se utilizó el dato de densidad del taxon (número de células $/ \mathrm{ml}$ ) y para las muestras de red solo la información de presencia/ausencia.

También se analizó el método de muestreo (red, botella y muestra viva), con el cual cada una de las especies pudo ser registrada, para conocer la eficiencia de captura de los distintos métodos de recolecta y la forma en que los diferentes taxa pudieron ser censados.

\section{RESULTADOS Y DISCUSIÓN}

Parámetros ambientales

Los valores obtenidos de la evaluación de los parámetros ambientales y biomasa por clorofila- $a$ en 8 sitios de colecta y varias profundidades, a partir de un $\mathrm{n}=589 \mathrm{ob}$ servaciones por parámetro, mostraron datos muy variables de temperatura, entre 19.3 y $31^{\circ} \mathrm{C}$, con los valores más altos $\left(>28^{\circ} \mathrm{C}\right)$ de junio a octubre (Fig. 2a). La salinidad osciló entre 29.5 a 38.3 , con los números más bajos en época de lluvias y hasta el inicio de las secas (julio a diciembre), encontrando dos picos de alta salinidad, uno en septiembre y otro en febrero 2010 (Fig. 2a), que coincidieron también con disminución de temperatura, lo que podría indicar la intrusión de agua menos cálida y más salina, producida posiblemente por una surgencia local. Las concentraciones de oxígeno disuelto oscilaron entre 0.79 a $11.19 \mathrm{mg} \mathrm{L}^{-1}$, con el dato más alto en octubre de 2010 y los más bajos en época de secas (Fig. 2b). Los valores de biomasa también fueron muy variables, entre $1 \times 10^{-3}$ a $46.28 \mathrm{mg} \mathrm{m}^{-3}$, con los máximos observados en la época seca (Fig. 2b). Respecto a los nutrientes, tanto los ortofosfatos $\left(2.2 \times 10^{-3}-1.29 \mathrm{mg} \mathrm{L}^{-1}\right)$, como los nitritos + nitratos $\left(4.9 \times 10^{-5}-27.6 \mathrm{mg} \mathrm{L}^{-1}\right)$, variaron considerablemente a lo largo del año; siendo los primeros más abundantes en la época de lluvia intensa, y los segundos aumentando gradualmente hacia finales del año, con los máximos en la época seca y menos cálida. Los silicatos: $2.2 \times 10^{-3}-0.5 \mathrm{mg} \mathrm{L}^{-1}$, presentaron valores constantes a lo largo del año (Fig. 2c), a semejanza del amonio: $5 \times 10^{-3}-0.48 \mathrm{mg} \mathrm{L}^{-1}$, que registró ligeros picos en el mes de octubre (al terminar las lluvias). El comportamiento general de los distintos nutrientes evaluados a lo largo del año, mostró bastante semejanza con los datos obtenidos por Rojas-Herrera et al. (2012); sin embargo en el presente estudio, los valores de ortofosfatos fueron más elevados que los de amonio, posiblemente como resultado de la alta precipitación pluvial recibida en 2010, en comparación con el promedio de la misma registrada en los 36 años anteriores, como lo muestra la figura $2 \mathrm{~d}$. Las altas concentraciones de ortofosfatos, también podrían ayudar a explicar los FAN de dinoflagelados ocurridos en el año 2010. 
Meave-del Castillo et al.: Ficoflora planctónica de la Bahía de Acapulco, México
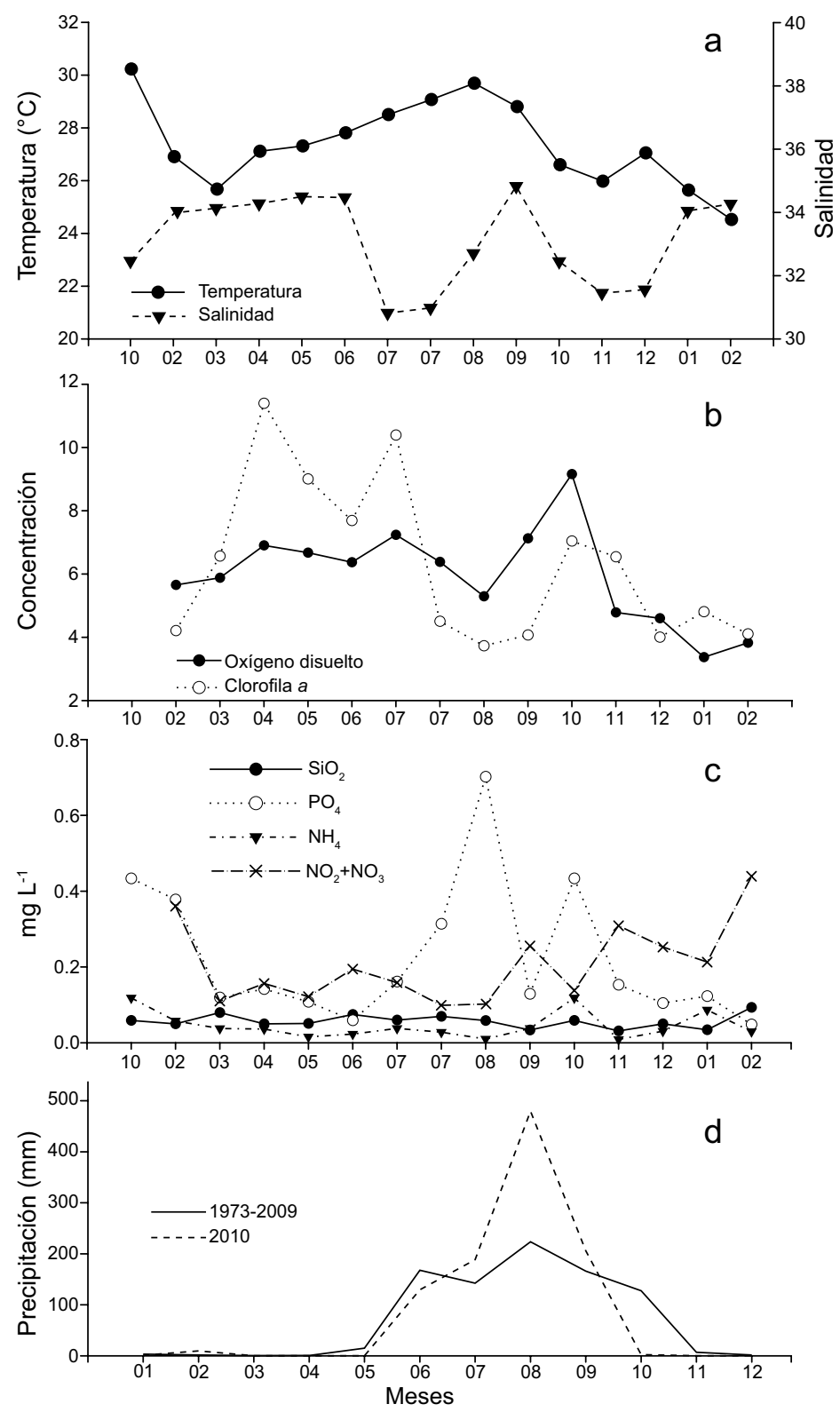

Figs. 2a-d. Gráficos de parámetros ambientales del ciclo febrero 2010 a febrero 2011 (incluido octubre 2009). a) Temperatura $\left({ }^{\circ} \mathrm{C}\right)$ y salinidad; b) Oxígeno disuelto $\left(\mathrm{ml} \mathrm{L}^{-1}\right)$ y biomasa $(\mathrm{Cl}-$ $a, \mathrm{mg} \mathrm{m}^{-3}$ ); c) Concentración en $\mathrm{mg} \mathrm{L}^{-1}$ de silicatos, ortofosfatos, amonio y nitratos + nitritos; d) Comparación de la precipitación pluvial $(\mathrm{mm})$ promedio mensual de 36 años y promedio mensual del año 2010. 
Composición específica

A partir de la revisión de 484 muestras (de red, botella y "vivas"), se identificó un total de 641 taxa de organismos planctónicos y ticoplanctónicos (Apéndice 1), pertenecientes a 8 divisiones, 14 clases, 49 órdenes, 89 familias y 168 géneros (Apéndice 2 y Cuadro 2). El grupo más diverso fue el de los dinoflagelados (Dinophyta) con 347 taxa, seguido de las diatomeas con 274 taxa (Apéndice 1). Ambos sumaron un porcentaje total de $96.8 \%$, por lo que la contribución de otros grupos microalgales a la flora planctónica de la Bahía de Acapulco, no parece ser importante. Sin embargo, el hecho de haber registrado momentos en la bahía de Acapulco, en los cuales fue abundante el protozoario Myrionecta rubra Jankowski, que se alimenta de dinoflagelados, que a su vez se nutren de fitoflagelados haptofitas (Johnson \& Stoecker,, 2005), así como al consorcio Leptocilindrus mediterraneus- Solenicola setigera que se nutre de picoplancton (Gómez, 2007), demuestra que al menos en ciertos momentos, las poblaciones de fitoflagelados y picoplancton deben ser importantes en la Bahía de Acapulco y que es necesario continuar realizando estudios florísticos específicos para esos grupos.

Los géneros de Bacillariophyta con mayor número de taxa (Apéndice 2) fueron: Chaetoceros (48), Rhizosolenia (13), Thalassiosira (11), Nitzschia (10) y Coscinodiscus (8), y de Dinophyta: Neoceratium-Ceratium (69), Protoperidinium (62), Gymnodinium (18), Dinophysis (16), Gonyaulax (16), Phalacroma (15), Prorocentrum (13), Oxytoxum (10), Gyrodinium (10) y Karenia (8). El género Neoceratium, fue erigido recientemente al encontrar un carácter taxonómico constante (cinco

Cuadro 2. Número de taxa por división, clase, orden, familia y género; y porcentaje por división. Arreglados por número de taxa.

\begin{tabular}{lccccccc}
\hline Grupos & Divisiones & Clases & Órdenes & Familias & Géneros & Taxa & $\%$ \\
\hline Dinophyta & 1 & 3 & 9 & 29 & 63 & 347 & 54.1 \\
Bacillariophyta & 1 & 3 & 29 & 46 & 88 & 274 & 42.7 \\
Heterocontophyta & 1 & 3 & 3 & 3 & 5 & 6 & 0.9 \\
Cyanophyta & 1 & 1 & 3 & 4 & 4 & 5 & 0.8 \\
Prymnesiophyta & 1 & 1 & 2 & 3 & 4 & 4 & 0.6 \\
Chlorophyta & 1 & 1 & 1 & 1 & 1 & 2 & 0.3 \\
Euglenophyta & 1 & 1 & 1 & 2 & 2 & 2 & 0.3 \\
Zoomastigophora & 1 & 1 & 1 & 1 & 1 & 1 & 0.2 \\
Totales & 8 & 14 & 49 & 89 & 168 & 641 & 100 \\
\hline
\end{tabular}


placas cingulares en lugar de seis) en las especies marinas que fue sustentado por estudios moleculares (Gómez et al., 2010); sin embargo en este trabajo 23 taxa infraespecificos (variedades y formas) fueron clasificados todavía dentro del género Ceratium, ya que formalmente no han sido transferidos a Neoceratium (de esta manera son citados como Neoceratium (Ceratium) en en los apéndices 1 y 2). A pesar de que todas las especies fitoplanctónicas reportadas por Rojas-Herrera et al. (2012) fueron también registradas en el presente estudio, llama la atención la diferencia en los valores de la riqueza consignados en ambas contribuciones (641 vs. 89), pero sobre todo el hecho de que en el total de las 484 muestras de red y botella analizadas en el presente estudio, Chaetoceros tetrastichon nunca llegó a ser dominante en la bahía de Acapulco, como lo mencionan tales autores.

La comparación del número de especies fitoplanctónicas de la Bahía de Acapulco con respecto a otras seis bahías ubicadas en el PM: Bahía de Manzanillo, Col. (404 taxa), Bahía de Banderas, Jal. (373 taxa), Bahía Mazatlán, Sin. (373 taxa), Bahía La Paz, B.C.S. (279 taxa), Bahía Concepción, B.C.S. (183 spp.) y Bahía Magdalena, B.C.S. (279 spp.), mostró que a pesar de su pequeño tamaño, Acapulco presenta una mayor riqueza, y lo más importante una mayor proporción de Dinophyta con relación a Bacillariophyta (Fig. 3a), lo cual concordó con los resultados florísticos mostrados por Rojas-Herrera et al. (2012). Fue interesante también observar que la diversidad fitoplanctónica de Acapulco incluso es mayor que la registrada para el Golfo de Tehuantepec y bastante cercana a la del Golfo de California (Fig. 3b); sobre todo porque ambos golfos son regiones mucho más extensas y con una dinámica oceanográfica fluctuante y dinámica, debido a la existencia de surgencias (LluchCota et al., 1997; Lluch-Cota, 2000).

A pesar del esfuerzo realizado para la identificación del fitoplancton, 57 taxa no pudieron ser determinados al nivel de especie, debido a la carencia de información de rasgos diagnósticos, o bien al hecho de que sus características no coincidieron con la descripción de algún taxon descrito revisado. Se presentan fotografías de 32 taxa no identificados, correspondientes a 16 Bacillariophyta: Achnanthes sp. (Fig. 4), Chaetoceros spp. (Figs. 5-7), Diploneis sp. (Fig. 8), Hantzschia sp. (Fig. 9); Haslea sp. (Fig. 10), Lyrella sp. (Fig. 12), Navicula sp. (Fig. 13), Petroneis sp. (Fig. 14), Pinnularia sp. (Fig. 15), Pleurosigma spp. (Figs. 16-17), Skeletonema sp. (Fig. 11) y Thalassiosira spp. (Figs. 18-19); 11 Dinophyta: Gonyaulax sp. (Fig. 20), Gymnodinium spp. (Figs. 22-24), Gyrodinium sp. (Fig. 25), Heterodinium sp. (Fig. 21), Scrippsiella sp. 1 (Figs. 27, 34), Scrippsiella sp. 2 (Figs. 35-36); Oxytoxum spp. (Figs. 28-29) y Phalacroma sp. (Fig. 30); una Cryptophyceae al parecer perteneciente al género Hillea sp. (Fig. 38); una (o dos) Primnesiophyta (o Hapto- 

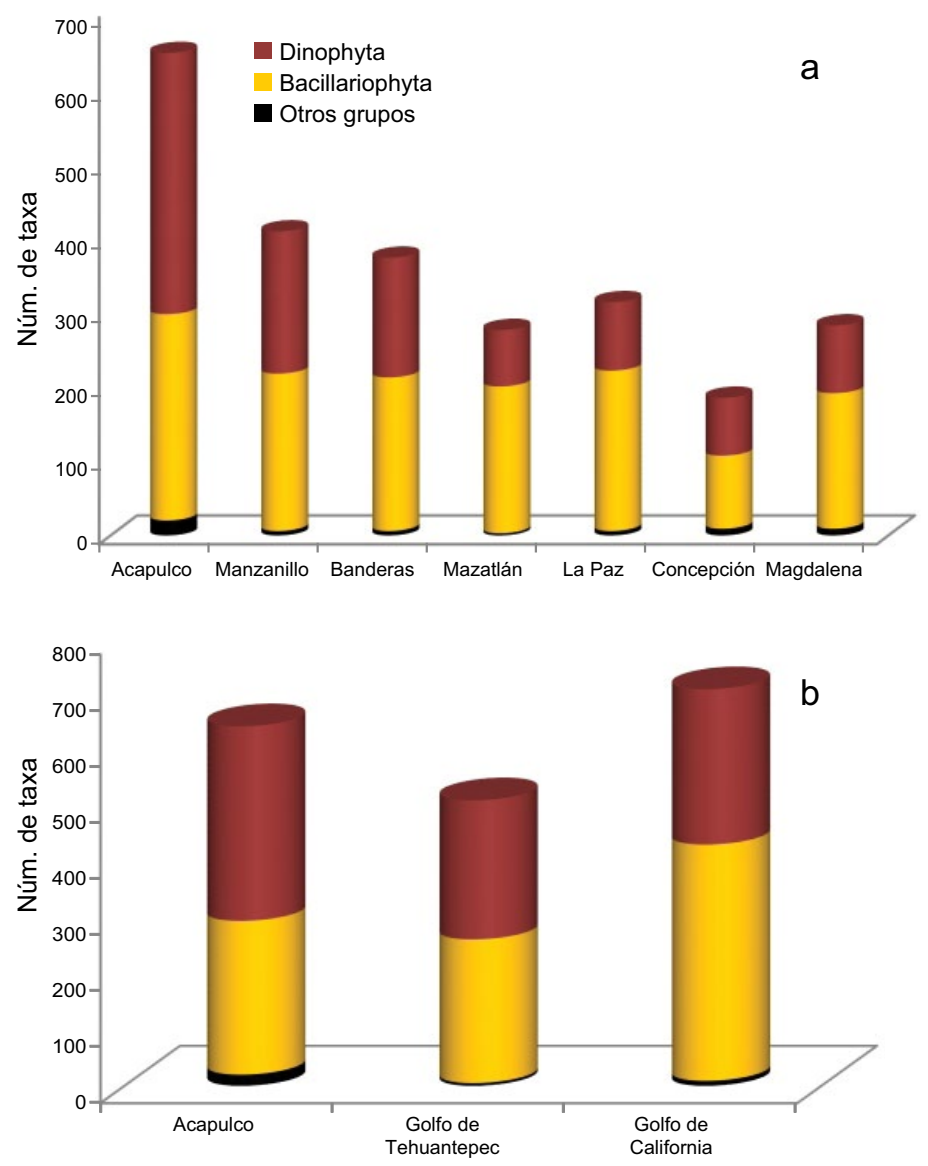

Figs. 3a-b. Comparación del número de taxa de fitoplancton encontrados en la Bahía de Acapulco con respecto al de diferentes localidades en el Pacífico Mexicano incluyendo las referencias de donde se tomó la información. a) Diferentes bahías del PM: Bahía de Manzanillo (Meave, 2006; Quijano-Seggia, et al., 2011), Bahía Banderas (Bravo-Sierra, 1999; Meave, 2006), Bahía Mazatlán (Cortés-Altamirano \& Pasten-Miranda, 1982a,b, 1985; Rojas-Trejo, 1984; Priego-Martínez, 1985), Bahía Concepción (Gárate-Lizárraga, 1991; Band-Schmidt, 2004; Morquecho \& Lechuga-Devéze, 2004; Estradas-Romero et al., 2009), Bahía La Paz (Gárate-Lizárraga \& Verdugo-Díaz, 2007; Gárate-Lizárraga \& Muñetón-Gómez, 2009; Gárate-Lizárraga et al., 2009; Gárate-Lizárraga \& González-Armas, 2011) y Bahía Magdalena (Gárate-Lizárraga, 1989, 1992; Gárate-Lizárraga et al., 2007, 2010). b) Golfos del PM: Golfo de Tehuantepec (Aké-Castillo, 1997; Aké-Castillo et al., 1999; Hernández-Becerril \& Bravo-Sierra, 2004a,b; Hernández-Becerril \& Diaz-Almeyda, 2006; Gárate-Lizárraga, 2008; Meave del Castillo y Hernández-Becerril, 1998) y Golfo de California (Licea et al., 1995; Moreno et al., 1996). 
phyta): Phaeocystis spp. (Figs. 30, 31-32); y tres Cyanophyta: Synechoccocus sp., Synechocystis sp. (Figs. 39-41) y Trichodesmium sp. (Fig. 26). Por último también una Zoomastigophora (Choanoflagellidae) faltó de ser determinada incluso al nivel genérico, ya que no fue posible observar con claridad la loriga (Fig. 37). Entre la ficoflora registrada, llamaron la atención dos clorofitas del género Pediastrum en la bahía de Acapulco, que claramente evidencian la entrada de arroyos de agua dulce a la bahía en la época de lluvia.

Dentro del total de especies encontradas en la Bahía de Acapulco, en el presente trabajo se ilustran también aquellas que ya habían sido reportados para el PM con anterioridad, pero de los cuales existen pocos registros: Planktoniella muriformis (Fig. 42, MEB), Achradina pulchra (Figs. 43-44), Cochlodinium convolutum (Fig. 45), C. fulvescens (Fig. 46), Erythropsidinium cf. agile (Figs. 47-48) y Gonyaulax sphaeroidea (Fig. 49).

Taxa registrados con problemática taxonómica

Con respecto a la presencia de especies raras e interesantes desde el punto de vista taxonómico, podemos resaltar a los dinoflagelados del género Alexandrium; uno de ellos presente en la bahía sobre todo en los meses de marzo y noviembre de 2010, mostró una morfología que corresponde con Alexandrium tamarense (Lebour) Balech (Figs. 50-52). Sin embargo, en la literatura se menciona que $A$. tamarense es de aguas frías $\left(10-16{ }^{\circ} \mathrm{C}\right.$; Itakura $\&$ Yamaguchi, 2001), y que está ampliamente distribuida en las regiones frías y templadas de ambos hemisferios de todos los océanos (Taylor et al., 2004), mientras que en Acapulco la temperatura fue cálida, variando entre 23 y $28^{\circ} \mathrm{C}$, en las distintas profundidades del periodo en el cual dicha especie se registró. Alexandrium tropicale Balech es bastante semejante a A. tamarense y se distribuye en aguas cálidas (Balech, 1995); sin embargo, descartamos ese nombre porque los organismos de Acapulco, presentaron la primera placa apical (1') más ancha, el poro ventral más pequeño y en posición menos basal, y la placa sulcal posterior ( $\mathrm{Sp}$ ) más alargada y sin poro de conexión, en comparación con A. tropicale. A pesar de lo anterior, sería conveniente no descartar totalmente la posibilidad de que corresponda a otro taxon tropical y no a $A$. tamarense, para lo cual sería necesario hacer un estudio más detallado de la morfología de los ejemplares mexicanos, incluyendo la secuenciación del ADN para confirmar la especie.

Otro taxon interesante fue Alexandrium fraterculus (Figs. 53-54), que aunque ya había sido registrada para el PM, se ilustra por vez primera; y por último otro identificado como $A$. cf. ostenfeldii (Figs. 55-56) que en general concordó bien con la forma y dimensiones de tal especie, e incluso al microscopio óptico se le observó 
Acta Botanica Mexicana 100: 405-487 (2012)
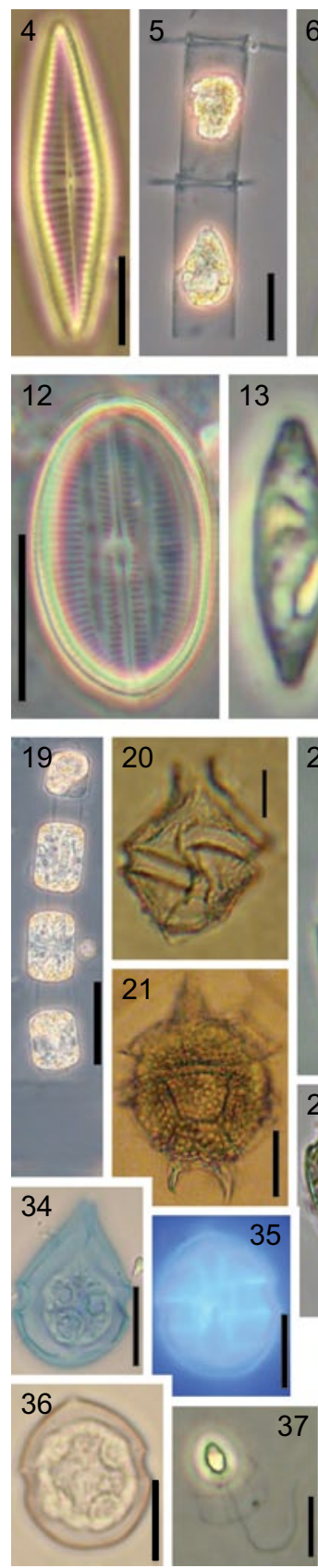
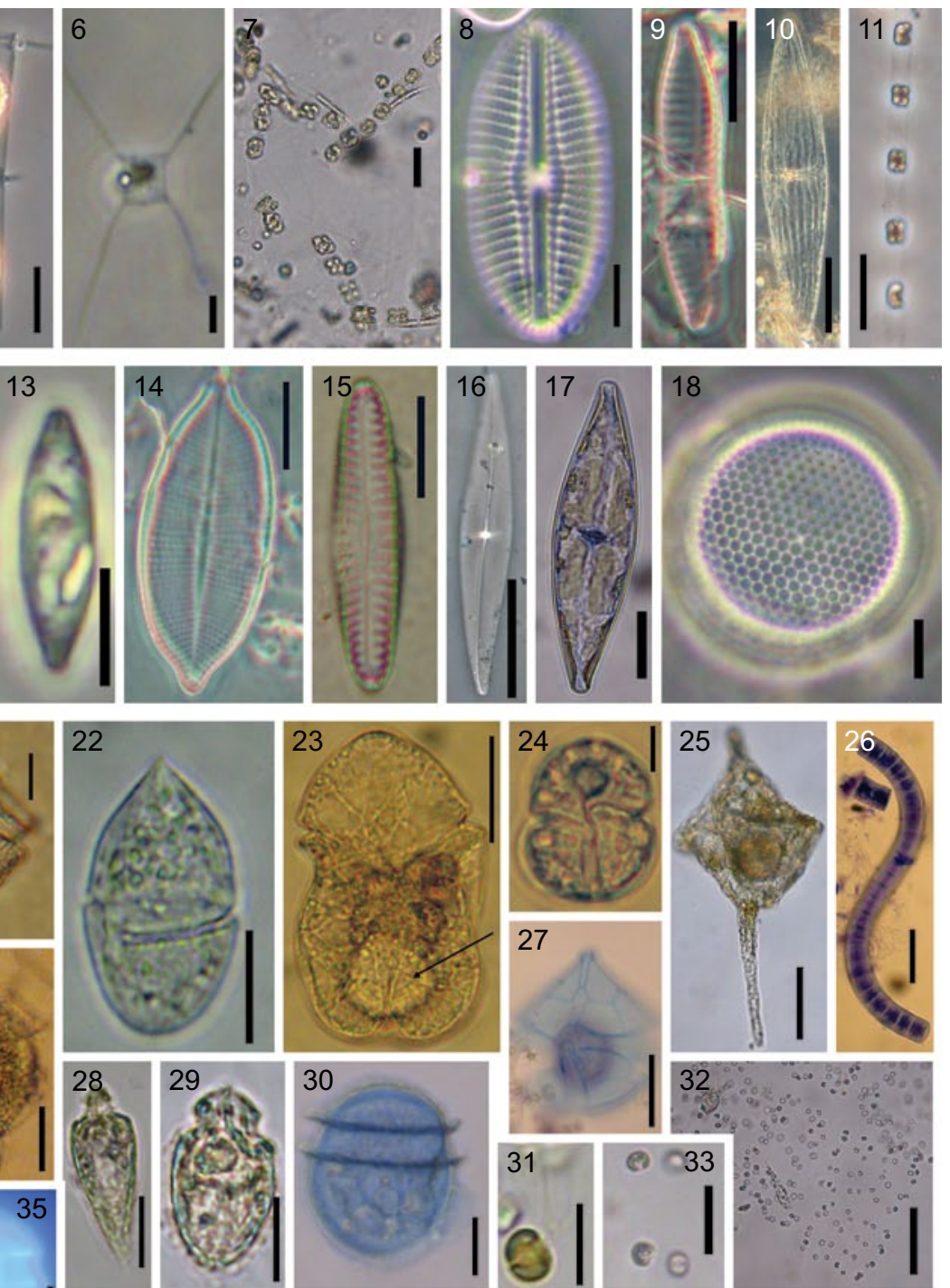

30
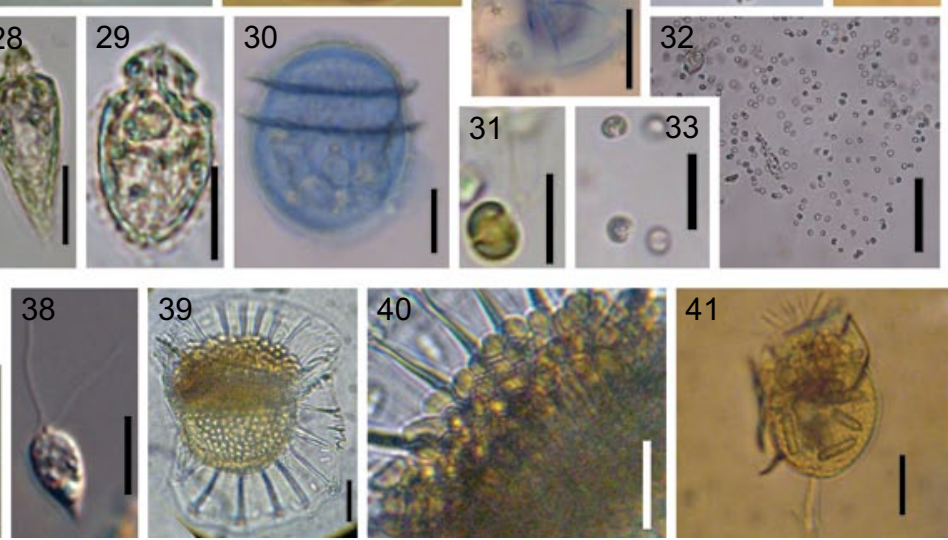

Pie de figura en página opuesta. 
el poro ventral de la placa 1' en posición basal, aunque no fue posible fotografiarlo. Sin embargo, surgió la duda de su identidad porque los organismos de Acapulco formaban cadenas con dos células, mientras que en la literatura se señala que $A$. ostenfeldii es solitario.

En este rubro podemos señalar también a Protoperidinium minusculum (Fig. 57), otro dinoflagelado tecado relacionado con $P$. bipes y entre los cuales hay incongruencias nomenclaturales: puesto que Balech (1988) no lo valida, mencionando que es sinónimo de $P$. bipes; y por el contrario Taylor (1976) reconoce a $P$. minusculum señalando a $P$. bipes como su sinónimo. Revisando la morfología de ambos taxa, se evidenció la posibilidad de que $P$. minusculum y $P$. bipes fueran especies distintas, puesto que $P$. minusculum es más pequeña, la forma de su teca es distinta a la de $P$. bipes (más alargada y con el cuerno apical más prolongado) y el cíngulo más ancho, todo lo cual concordó con los ejemplares de Acapulco. Para confirmar tal hipótesis, necesariamente habrá que hacer un estudio detallado de las placas de la teca, porque la Sección Minuscula, a la cual pertenece esta especie, presenta solo 6 placas precingulares, en lugar de 7 como ocurre en la mayoría de los Protoperidinium (Taylor, 1976).

Figs. 4-41. Taxa identificados solo al nivel de género, ejemplares vistos bajo el microscopio óptico, excepto Fig. 35. con microscopio de fluorescencia. Fig. 4. Achnanthes sp., vista valvar. Fig. 5. Chaetoceros sp. 1, vista conectiva. Fig. 6. Chaetoceros sp. 2, vista conectiva. Fig. 7. Chaetoceros sp. 3, masa formada por cadenas. Fig. 8. Diploneis sp., vista valvar. Fig. 9. Hantzschia sp., vista valvar. Fig. 10. Haslea sp., vista valvar. Fig. 11. Skeletonema sp. 2, vista conectiva. Fig. 12. Lyrella sp., vista valvar. Fig. 13. Navicula sp. 1, vista valvar. Fig. 14. Petroneis sp., vista valvar. Fig. 15. Pinnularia sp., vista valvar. Fig. 16. Pleurosigma sp.1, vista valvar. Fig. 17. Pleurosigma sp. 2, vista valvar. Fig. 18. Thalassiosira sp. 1, vista valva. Fig. 19. Thalassiosira sp. 2, vista conectiva. Fig. 20. Gonyaulax sp., vista ventral. 21. Heterodinium sp., vista dorsal. Fig. 22. Gymnodinium sp. 1, vista lateral. Fig. 23. Gymnodinium sp. 2, vista ventral. Fig. 24. Gymnodinium sp. 3, vista ventral. Fig. 25. Gyrodinium sp., vista lateral. Fig. 26. Trichodesmium sp., filamento. Figs. 27, 34. Scrippsiella sp. 1, vista ventral. Fig. 28. Oxytoxum sp. 1. Fig. 29. Oxytoxum sp. 2. Fig. 30. Phalacroma sp., vista lateral derecha. Figs. 31-33. Phaeocystis spp.: Fig. 31. Célula flagelada con haptonema (¿zooide?), Figs. 32-33. Estadio colonial, Fig. 32. Células embebidas en cenobio, Fig. 33. Conjunto de células formando tétradas dentro de cenobio. Figs. 35-36. Scrippsiella sp. 2, vista ventral. Fig. 37. Choanoflagelado. Fig. 38. Cryptophyceae: cf. Hillea sp. Figs. 39-41. Feosomas: Figs. 39-40. Synechocystis sp. en velos cingulaes de Ornithocercus; Fig. 41. Synechococcus sp. sobre el cuerpo de Histioneis y el velo sulcal de Ornithocercus. Escalas: Figs. 5, 18, 24, $31=5 \mu \mathrm{m}$; Figs. 4, 6, 8-9, 12-15, 20, 22, 27-30, 34-38, 40-41 = $10 \mu \mathrm{m}$; Figs. 7, 11, 17, 21, $23,26,33,39=20 \mu \mathrm{m}$; Figs. 10, 16, 19, 25, $32=50 \mu \mathrm{m}$. 
Otro dinoflagelado desnudo con problemática taxonómica fue Erythropsidinium cf. agile (Figs. 47-48). En la actualidad se ha reconocido que E. agile tiene una morfología muy variable. Originalmente Kofoid y Swezy (1921) reconocieron seis especies distintas con cierta semejanza a $E$. agile, considerando la forma del cuerpo, la presencia o no de estilete en la punta del pistón, la conformación del oceloide, el color del citoplasma y del cuerpo pigmentario, la forma del núcleo y otros organelos internos. Posteriormente, Elbrächter (1979) observó que todos esos morfotipos eran variaciones de un mismo taxon, de acuerdo con el desarrollo del ciclo de vida y fueron considerados sinónimos. Los organismos encontrados en Acapulco se asemejaron más a la forma "E. pavillardi“, descrita por Kofoid y Swezy (1921) y llamó la atención que, considerando todos los sinónimos para obtener el intervalo de medida de la especie (con datos de Kofoid \& Swezy, 1921; Gómez, 2008 y Gárate-Lizárraga et al., 2010), los ejemplares de Acapulco, tuvieran aún menores dimensiones, tanto en la talla total $(88-213 \mu \mathrm{m}, x=142 \mu \mathrm{m}$ vs. $120-590 \mu \mathrm{m})$, como en la talla del cuerpo celular (31-90 $\mu \mathrm{m}, x=61.8 \mu \mathrm{m}$ vs. $55-140 \mu \mathrm{m})$, del pistón $(37-135, x=78.3 \mu \mathrm{m}$ vs. $55-450 \mu \mathrm{m})$, e incluso del diámetro de los lentes del oceloide (10-23 $\mu \mathrm{m}, x=14.7 \mu \mathrm{m}$ vs. 17-32 $\mu \mathrm{m}$ ). Además en los ejemplares de Acapulco, el pistón nunca llegó a tener una extensión siquiera del doble del cuerpo celular, mientras que Kofoid y Swezy (1921) señalaron que en los Erythropsidinium, generalmente este era cuatro veces más largo que el cuerpo; y Gómez (2008) observó un ejemplar de E. agile con una longitud del pistón veinte veces más largo que el cuerpo. Además, en los ejemplares de Acapulco el oceloide tuvo una posición más basal de lo que es mencionado para E. agile (Fig. 48). Descartamos el hecho de que las diferencias encontradas pudieran explicarse por efectos de la alteración de la morfología, debido a la fijación con lugol, porque las fotografías de ejemplares de E. agile, también fijados en lugol, presentadas por Gómez (2008), son bastante diferentes de los de Acapulco.

Dentro de la División Primnesiophyta, en Acapulco se observaron organismos identificados como miembros de género Phaeocystis (Figs. 30-32), cuya especie no pudo ser determinada, constituyendo dos morfotipos, como ocurre en la mayoría de los representantes del género: a) cenobios mucilaginosos (Fig. 32-33), con células inmóviles embebidas, distribuidas de manera dispersa y más o menos ordenada, entre las cuales se observaban conjuntos de células formando tétradas (Fig. 33), y b) formas unicelulares móviles (Fig. 31), subesféricas (3-5x3-4 $\mu \mathrm{m}$ ), biflageladas con el haptonema más corto $(2.2-2.5 \mu \mathrm{m})$ que los flagelos $(6 \mu \mathrm{m})$. Tanto las células embebidas en las colonias (Fig. 32), como las solitarias móviles (zooides; Fig. 31), tuvieron forma de corazón o arriñonada, con el antápice redondeado y la porción apical muy escavada, así como similares dimensiones (3-5 $\mu \mathrm{m} \times 3-4 \mu \mathrm{m})$, coincidentes con 
$P$. cordata. Sin embargo, la identificación quedó pendiente porque Zingone et al. (1999) señalan que $P$. cordata no forma el estado colonial. Aunque los organismos de Acapulco también mostraron semejanzas con P. pouchetti (Hariot) Legerheim, esa opción igualmente se descartó, porque esta última es considerada una especie de aguas frías, con un intervalo de adaptación de 2 a $14{ }^{\circ} \mathrm{C}$ y un óptimo en $8{ }^{\circ} \mathrm{C}$ (Moestrup \& Thompsen, 2004), en tanto que en Acapulco se le colectó en agua muy cálida $\left(26-28^{\circ} \mathrm{C}\right)$. Para conocer la naturaleza de estas Primenesiophyta, habrá que hacer estudios más específicos de su morfología e incluso intentar cultivarlas, para por una parte, conocer si se trata de dos estadios de la misma especie y también para tener suficiente material para su estudio con microscopía electrónica, toda vez que las delicadas escamas orgánicas que cubren su pared, constituyen caracteres taxonómicos más confiables (Zingone et al., 1999).

Taxa interesantes registrados en la bahía de Acapulco

Entre los taxa de dinoflagelados encontrados en la Bahía de Acapulco, un hallazgo interesante fue el de Neoceratium dens (Fig. 58), exclusivamente a partir de mayo de 2010, con abundancias de 120-200 Céls. $\mathrm{L}^{-1}$ a $5 \mathrm{~m}$ de profundidad. Hasta hace algunos años esta especie se conocía solamente de la región del Indopacífico y fue encontrada recientemente (2009) en el Golfo de California, México (GárateLizárraga, 2009), lo que podría evidenciar su translocación por agua de lastre de buques cargueros o bien su acarreo por corrientes que han cambiado sus patrones de movimiento debido al cambio climático global (Gárate-Lizárraga, 2009). Su hallazgo en Acapulco indica su alta capacidad de dispersión, facilitada posiblemente por su acarreo hacia el sur, a través de la Corriente de California y con ello la probabilidad de que ya esté distribuida en todo el Pacífico Mexicano.

Otro aspecto remarcable en los resultados taxonómicos de la ficoflora de Acapulco, fue la presencia de varias especies de dinoflagelados desnudos del género Karenia, incluyendo la posibilidad de tener a K. brevis (Fig. 59) en el PM, a pesar de que se le ha señalado como endémica de la región del Golfo de México; sobre todo considerando que existen registros de la especie en el Pacífico Occidental (Japón y Nueva Zelanda; Taylor et al., 2004).

Entre las Euglenophyta, llamó la atención el hallazgo de una especie perteneciente al grupo Serpentes, dentro del género Euglena (Huber-Pestalozzi, 1955), de color verde, con movimiento euglenoideo intenso, con un solo flagelo que se pierde con facilidad, semejante a $E$. pascheri (Figs. 60-64), aunque de mayores dimensiones que lo reportado por Huber-Pestalozzi (1955) y por Wolowski y Hindák (2005): 34.5-58 $\mu \mathrm{m} \times 3.8-9.0 \mu \mathrm{m}$ vs. $17-70 \mu \mathrm{m} \times 6.0-32 \mu \mathrm{m}$. En la literatura se menciona que 

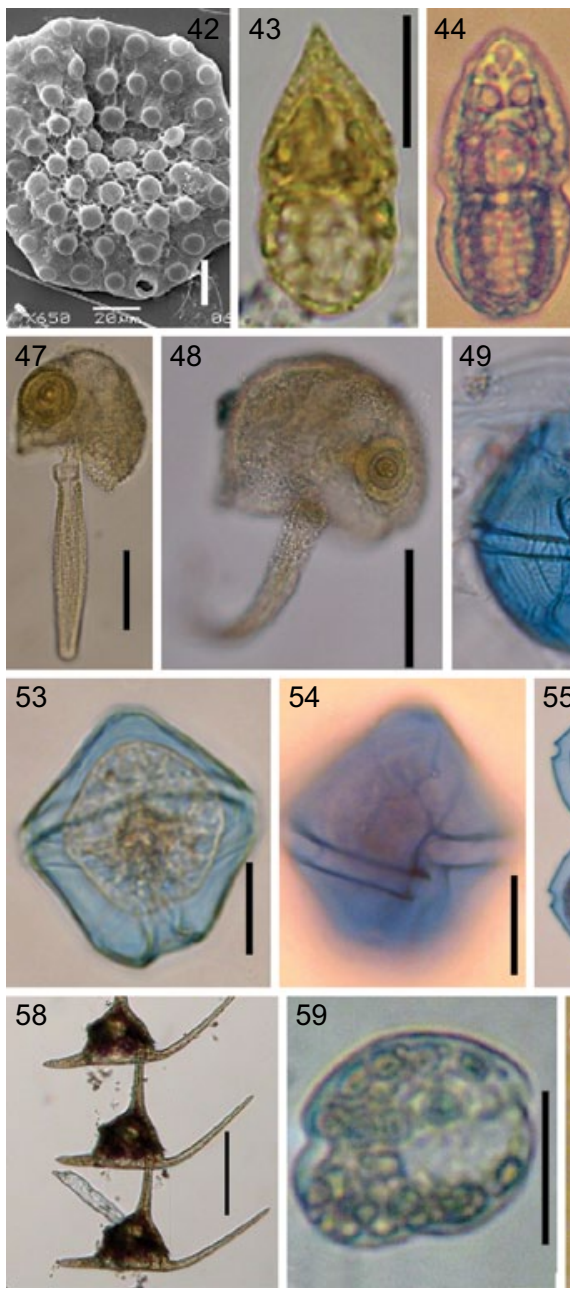
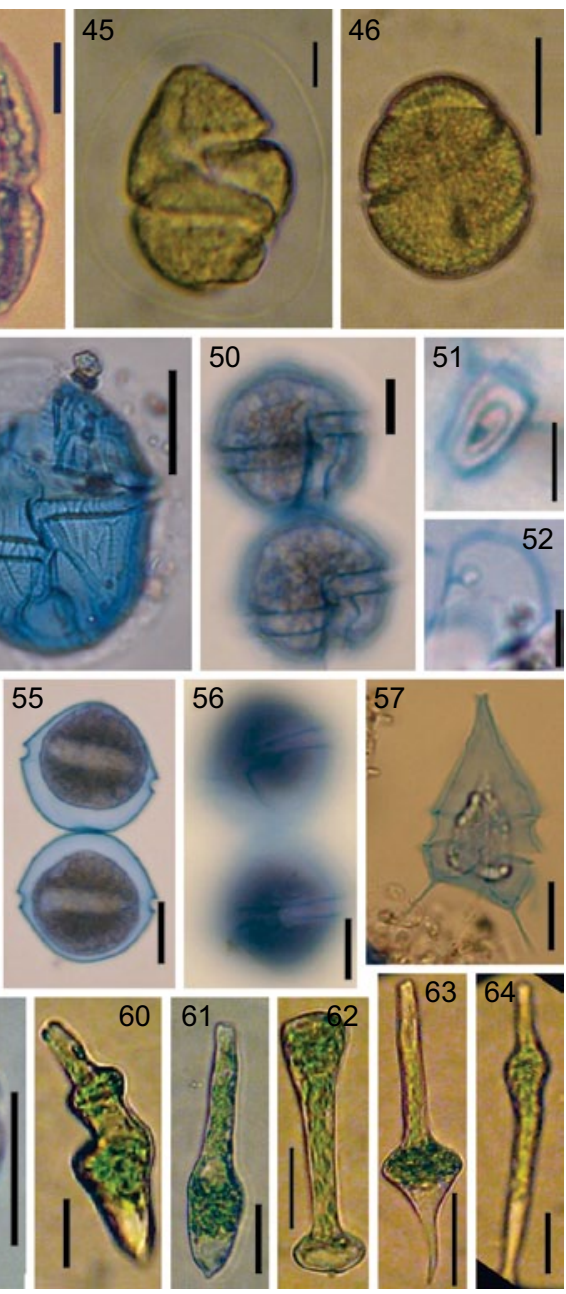

Figs. 42-64. Taxa interesantes taxonómicamente, ejemplares vistos bajo el microscopio óptico, excepto la figura 42, tomada con microscopio electrónico de barrido. Fig. 42. Planktoniella muriformis, colonia. Figs. 43-44. Achradina pulchra, vista dorsal. Fig. 45. Cochlodinium convolutum, vista ventral. Fig. 46. C. fulvecens, vista lateral. Figs. 47-48. Erythropsidinium cf. agile, vista ventral y vista lateral derecha. Fig. 49. Gonyaulax sphaeroidea, vista ventral. Fig. 50-52. Alexandrium cf. tamarense: Fig. 50. Cadena de dos células en vista ventral, Fig. 51. Complejo poro apical, Fig. 52. Placa sulcal inferior con poro antapical. Figs. 53-54. A. fraterculus: Fig. 53. Vista dorsal, Fig. 54. Vista ventral con placa 1'. Fig. 55-56. A. cf. ostenfeldii: Fig. 55. Vista dorsal, Fig. 56. Vista ventral. Fig. 57. Protoperidinium minusculum, vista ventral. Fig. 58. Neoceratium dens, vista dorsal. Fig. 59. Karenia cf. brevis, vista ventral. Figs. 60-64. Euglena pascheri, secuencia de movimiento metabólico. Escalas: Fig. 45, 51-52 $=5 \mu \mathrm{m}$; Figs. 43-44, 46, 50, 53-54, $57=10 \mu \mathrm{m}$; Figs. 42, 49, 55-56, 59-64 = $20 \mu \mathrm{m}$; Figs. $47-48=50 \mu \mathrm{m}$; Figs. $58=100 \mu \mathrm{m}$. falta escala para $51,52,54,56,57$. 
E. pascheri es indicadora de la presencia de aguas negras en los cuerpos de agua, incluso dado que es tolerante a condiciones anóxicas, puede vivir bajo condiciones microaerofílicas, causadas por la descomposición de la materia orgánica (Ligeza \& Wilk-Wozniak, 2011). Este taxon estuvo presente en la bahía a lo largo de todo el año, sin embargo sus abundancias indicaron su asociación con la localidad de "La Naval", sitio que tiene el mayor tiempo de resilencia en la bahía, debido a que sufre un menor intercambio de agua con las corrientes que llegan desde mar abierto, por lo que posiblemente llegue a acumular una concentración de materia orgánica mayor.

\section{Nuevos registros}

De las 641 taxa registrados en la Bahía de Acapulco, 38 son nuevos registros para el Pacífico Mexicano (Cuadro 3): 36 dinoflagelados (94.7\%) y 2 diatomeas: Delphineis minutissima (Fig. 65) у Pseudoguinardia recta (Fig. 66). Entre los 38, 18 no se habían reconocido en aguas mexicanas (Cuadro 3). De los nuevos registros de dinoflagelados, solo cinco son tecados: cuatro Gonyaulacales, uno perteneciente a la familia Heterodiniaceae: Heterodinium blackmanii (Fig. 91), y tres a Ceratiaceae: Neoceratium biceps (Fig. 96), Neoceratium (Ceratium) carriense var. volans (Fig. 104) y Neoceratium (Ceratium) vultur f. japonicum (Fig. 100), y un representante de Pyrocystaceae: Pyrocystis gerbaultii (Fig. 99). Los 32 restantes son dinoflagelados desnudos o atecados, colectados principalmente con botella $u$ observados en muestras vivas, pertenecientes a las familias Gymnodiniaceae: Amphindinium (Figs. 6769), Cochlodinium (Fig. 72), Gymnodinium (Figs. 75-80, 85-88), Gyrodinium (Figs. 82-89), Karenia (Figs. 92-93), Takayama cf. cladochroma (Fig. 98) y Torodinium teredo (Fig. 95), Polykrikaceae: Polykrikos gerbaultii (Fig. 97), Warnowiaceae: $\mathrm{Ce}-$ ratoperidinium yeye (Fig. 70), Greuetodinium cylindricum (Figs. 73-74), Nematodinium torpedo (Fig. 94), Warnowia (Figs. 102-103); un taxon incertae sedis (Gynogonadinium aequatoriale, (Fig. 81); así como un representante del orden Noctilucales, familia Leptodiscaceae (Scaphodinium miriabile), y el parásito Chytriodinium affi$n e$, del orden Blastodiniales (Fig. 71).

Caracterización biológica de la comunidad fitoplanctónica

Con respecto a la caracterización de la comunidad fitoplanctónica, en cuanto al nivel de organización de los taxa registrados, se encontró que la mayoría (325 taxa, 51\%) son unicelulares flagelados (móviles) y 176 (27\%) unicelulares no flagelados. Otro nivel de organización importante en la bahía son las cadenas o filamentos (136 taxa, 21\%); por el contrario solo cinco de las especies registradas son cenobiales o colonias, con las células embebidas en una matriz mucilaginosa (Fig. 
Acta Botanica Mexicana 100: 405-487 (2012)
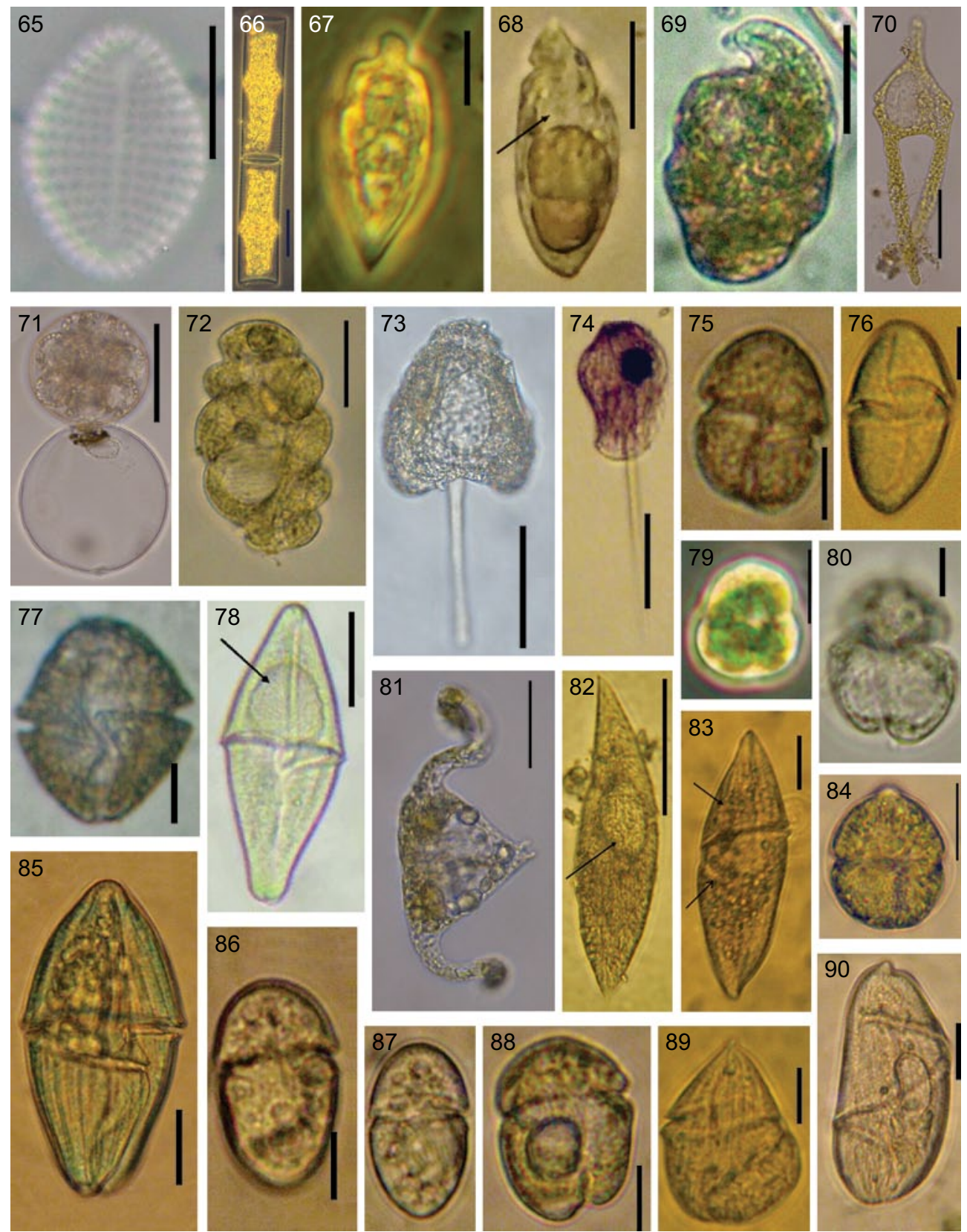

89
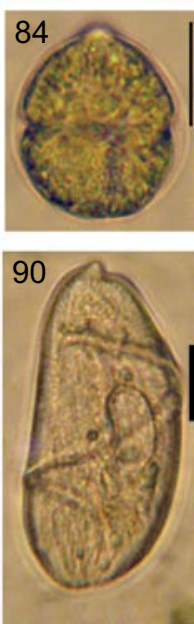

Pie de figura en página opuesta. 
105a), las diatomeas: Chaetoceros sp. 1 (Fig. 7) y Planktoniella muriformis (Fig. 42) y la primensiofita Phaeocystis spp. (Figs. 31-33) y dos colonias-consorcios de Pedistraum spp. La mayor parte de los taxa encontrados en la bahía tienen una nutrición autótrofa (423 taxa, 66\%), seguidos por los heterótrofos (115 taxa, 18\%) y los mixótrofos (104 taxa, 16\%) Fig. 105b. Con respecto al hábito, los de la mayoría (556 taxa, 87\%) son planctónicos, con 11\% (73 taxa) de organismos ticoplanctónicos y un número bajo (13 taxa, 2\%) de algas diafóricas (Rojas-Herrera et al., 2012), los cuales han sido reportados como planctónicos y/o bentónicos (Fig. 105c).

En cuanto a su distribución mundial, $48 \%$ de los taxa están reportados como cosmopolitas, 201 (31\%) son de afinidad tropical, 85 (13\%) de afinidad templada-fría y 14 taxa (2\%) con una distribución subtropical (Fig. 105d). Los organismos conocidos como cosmopolitas, en su mayoría fueron dinoflagelados (193 taxa, 30\%), mientras que las diatomeas de tan amplia distribución (112 taxa) representaron solo el $17 \%$ del total, el $1 \%$ restante corresponde a otros grupos algales. La mayoría de los 48 taxa reportados con distribución tropical-subtropical fueron diatomeas (54\%), en tanto los dinoflagelados representaron $46 \%$.

Por último, en relación a la caracterización de la comunidad microalgal de la Bahía de Acapulco, se registró que la mayoría de los taxa identificados en la bahía $(376,59 \%)$, tienen una afinidad nerítica-oceánica. Los datos particulares por grupo mostraron que $41 \%$ de las diatomeas registradas en Acapulco y $42 \%$ de los dinoflagelados son neríticos (Figs. 105f-g) y que del total, solo 146 taxa (23\%) son

Figs. 65-90. Nuevos registros para el Pacífico Mexicano, ejemplares vistos bajo el microscopio óptico, excepto la figura 65, tomada con microscopio electrónico de barrido. Fig. 65. Delphineis minutissima, vista valvar. Fig. 66. Pseudoguinardia recta, dos células en vista conectiva. Fig. 67. Amphidinium acutissimum. Fig. 68. A. extensum (flecha señala el núcleo, MO). Fig. 69. A. gibbosum. Fig. 70. Ceratoperidinium yeye. Fig. 71. Chytriodinium affine, trofonte adherido a un huevo de copépodo. Fig. 72. Warnowia juno, vista ventral. Figs. 73-74. Greuetodinium cylindricum: Fig. 73. Vista ventral, Fig. 74. Vista lateral derecha. Fig. 75. Gymnodinium aestuariale, vista ventral. Fig. 76. G. allophron, vista ventral. Fig. 77. G. aureolum, vista ventral. Fig. 78. G. aureum, vista ventral, la flecha señala el núcleo. Fig. 79. G. cf. grammaticum. Fig. 80. G. parvulum, vista ventral. 81. Gynogonadinium aequatoriale, vista lateral. Fig. 82. Gyrodinium acutum, vista dorsal (flecha señala el núcleo, MO). Fig. 83. G. britannicum, vista dorsal. Fig. 84. G. foliaceum, vista dorsal. Fig. 85. Gymnodinium translucens, vista lateral. Figs. 86-87. G. ravenescens, vista ventral y vista lateral respectivamente. Fig. 88. G. sphaericum, vista ventral. Fig. 89. Gyrodinium pepo, vista dorsal. Fig. 90. G. cf. ochraceum, vista ventral. Escalas: Figs. $65,75,80=5 \mu \mathrm{m}$; Figs. 67-69, 76-79, 81, 86-88 $=10 \mu \mathrm{m}$; Figs. $66,70,72,81,83-85,89-90=20 \mu \mathrm{m}$; Figs. $71,73-74,82=50 \mu \mathrm{m}$. 
Cuadro 3. Nuevos registros para México y el Pacífico Méxicano (PM).

\begin{tabular}{|c|c|c|c|}
\hline \multicolumn{4}{|l|}{ Bacillariophyta } \\
\hline Delphineis minutissima (Hustedt) Simonsen & PM & & Fig. 65 \\
\hline Pseudoguinardia recta von Stosch & PM & México & Fig. 66 \\
\hline \multicolumn{4}{|l|}{ Dinophyta } \\
\hline Amphidinium acutissimum Schiller & PM & & Fig. 67 \\
\hline A. extensum Wulff & PM & México & Fig. 68 \\
\hline A. gibbosum (Maranda et Shimizu) Jørgensen et Murray & PM & México & Fig. 69 \\
\hline Ceratoperidinium yeye Margalef & PM & México & Fig. 70 \\
\hline Chytriodinium affine (Dogiel) Chatton & PM & México & Fig. 71 \\
\hline Cochlodinium cf. archimedes (Pouchet) Lemmermann & PM & & Fig. 72 \\
\hline Greuetodinium cylindricum (Greuet) Loeblich III & PM & & Figs. $73-74$ \\
\hline Gymnodinium aestuariale Hulburt & PM & México & Fig. 75 \\
\hline G. allophron Larsen & PM & México & Fig. 76 \\
\hline G. aureolum (Hulburt) G. Hansen & PM & & Fig. 77 \\
\hline G. aureum Kofoid et Swezy & PM & México & Fig. 78 \\
\hline G. cf. grammaticum (Pouchet) Kofoid et Swezy & PM & México & Fig. 79 \\
\hline G. parvulum Schütt & PM & México & Fig. 80 \\
\hline G. ravenescens Kofoid et Swezy & PM & & Figs. $86-87$ \\
\hline G. sphaericum Calkins & PM & México & Fig. 88 \\
\hline G. translucens Kofoid et Swezy & PM & México & Fig. 85 \\
\hline Gynogonadinium aequatoriale Gómez & PM & México & Fig. 81 \\
\hline Gyrodinium acutum (Schütt) Kofoid et Swezy & PM & & Fig. 82 \\
\hline G. britannicum Kofoid et Swezy & PM & México & Fig. 83 \\
\hline G. foliaceum Kofoid et Swezy & PM & México & Fig. 84 \\
\hline G. cf. ochraceum Kofoid et Swezy & PM & & Fig. 90 \\
\hline G. pepo (Schütt) Kofoid et Swezy & PM & & Fig. 89 \\
\hline Heterodinium blackmanii (Murray et Witting) Kofoid & PM & & Fig. 91 \\
\hline Karenia asteriochroma De Salas, Bolch et Hallegraeff & PM & México & Fig. 92 \\
\hline K. brevisulcata (Chang) Hansen et Moestrup & PM & & Fig. 93 \\
\hline Nematodinium torpedo Kofoid et Swezy & PM & & Fig. 94 \\
\hline $\begin{array}{l}\text { Neoceratium biceps (Claparède et Lachmann) Gómez, } \\
\text { Moreira et López-García }\end{array}$ & PM & & Fig. 96 \\
\hline $\begin{array}{l}\text { Neoceratium (Ceratium) carriense var. volans (Cleve) } \\
\text { Jörgensen }\end{array}$ & PM & & Fig. 104 \\
\hline N. (C.) vultur f. japonicum (Schröder) Wood & PM & & Fig. 100 \\
\hline
\end{tabular}


Cuadro 3. Continuación.

\begin{tabular}{|c|c|c|c|}
\hline Polykrikos kofoidii Chatton & PM & & Fig. 97 \\
\hline Pyrocystis gerbaultii Pavillad & PM & México & Fig. 99 \\
\hline Scaphodinium miriabile Margalef & PM & México & Fig. 101 \\
\hline $\begin{array}{l}\text { Takayama cf. cladochroma (Larsen) de Salas, Bolch et } \\
\text { Hallegraeff }\end{array}$ & PM & México & Fig. 98 \\
\hline Torodinium teredo (Pouchet) Kofoid et Swezy & PM & & Fig. 95 \\
\hline Warnowia juno (Schütt) Schiller & PM & & Fig. 102 \\
\hline W. maxima (Kofoid et Swezy) Lindemann & PM & & Fig. 103 \\
\hline
\end{tabular}

considerados como netamente oceánicos (Fig. 105e), por lo que para ambos grupos los organismos oceánicos representaron menos de $25 \%$ del total, entre los cuales se pueden citar los siguientes: Chaetoceros aequatorialis, C. messanensis, Climacodium frauenfeldianum, Gossleriella tropica, Haslea gigantea, H. wawrikae, Helicotheca tamesis, Rhizosolenia castracanei, Stellarima stellaris, Thalassiosira hendeyi, Asterodinium gracile, Corythodinium diploconus, C. elegans, Erythropsidinium cf. agile, Gynogonadinium aequatoriale, Histioneis hyalina, Kofoidinium splendens, $K$. velelloides, Scaphodinium miriabile y Spatulodinium pseudonoctiluca.

Por otra parte, la ficoflora encontrada en Acapulco es netamente marina (98.6\%), con un porcentaje bajo de taxa dulceacuícolas $(2,0.3 \%)$ y siete $(1.1 \%)$ salobres: Aulacoseira granulata, Cocconeis scutellum, Entomoneis alata, E. gigantea, Nitzschia gandersheimiensis, N. sigma y Thalassionema nitzschioides var. claviformis (Fig. 105h).

De los 38 nuevos registros para el Pacífico mexicano encontrados en la bahía de Acapulco, 37 correspondieron a especies netamente planctónicas y el restante es ticoplanctónico (Delphineis minutissima, Fig. 65); 14 son de afinidad nerítica, ocho oceánicos y del resto no se sabe con certeza; 15 (todos dinoflagelados) tienen una distribución conocida de aguas frías a templadas, seis son netamente tropicales (5 dinoflagelados y 1 diatomea), una: Gymnodinium aureolum (Fig. 77), es cosmopolita, exceptuando los polos, y otra: Takayama cf. cladochroma (Fig. 98), es subtropical (Apéndice 1).

Relaciones biológicas

A través del estudio florístico, también se registraron relaciones de simbiosis entre cianofitas coccoides o feosomas: Synechococcus sp. (Figs. 39 y 41) y Synecho- 

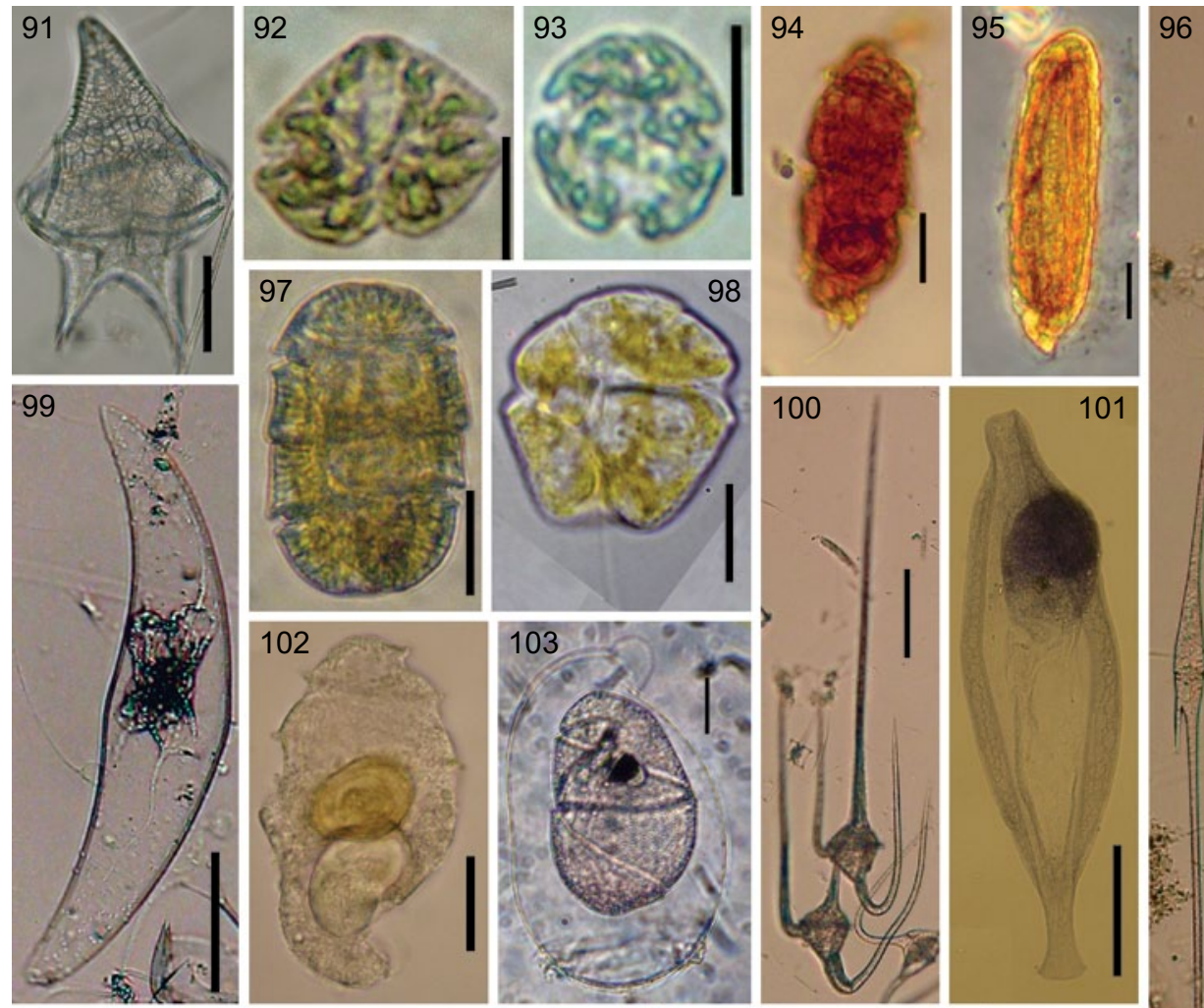

\section{0}
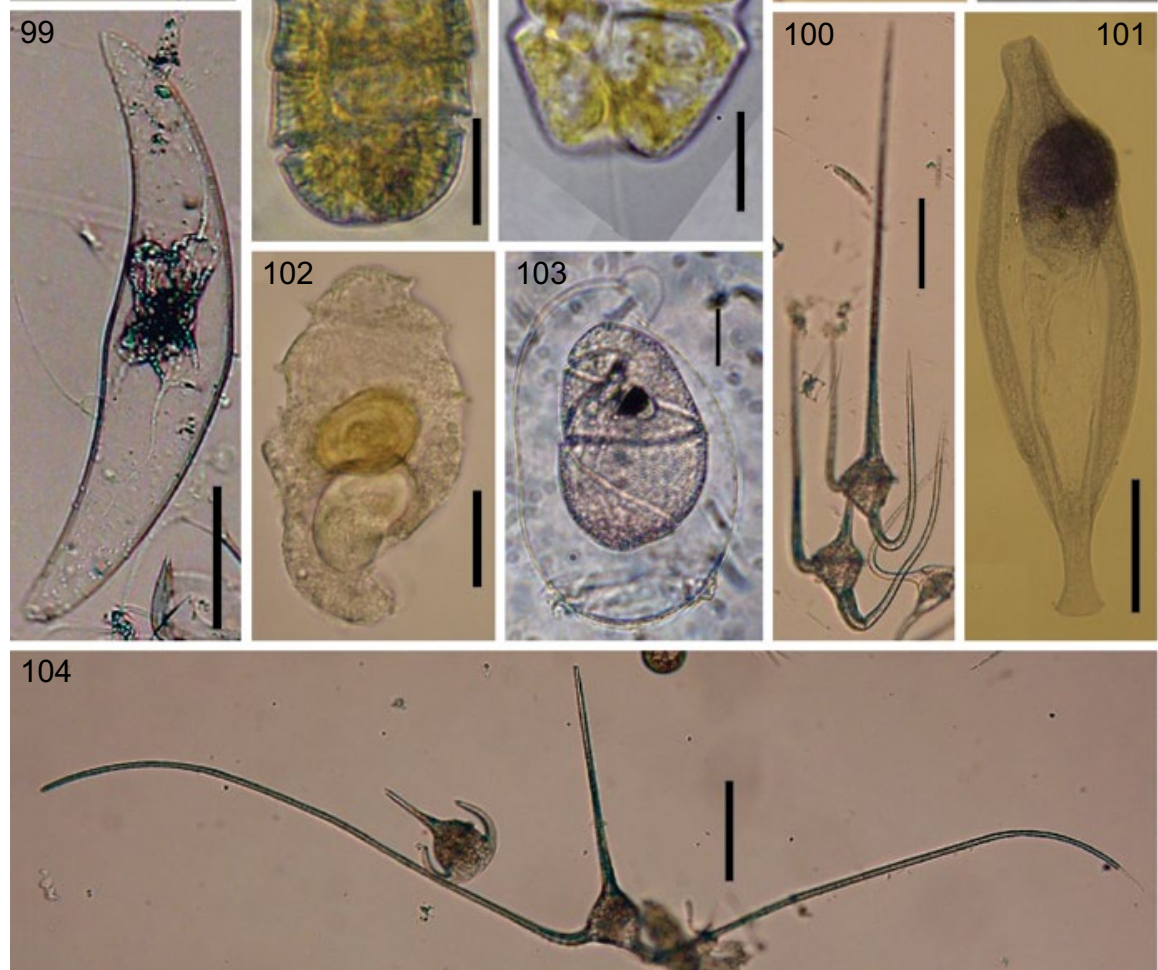

Figs. 91-104. Nuevos registros para el Pacífico Mexicano, ejemplares vistos bajo el microscopio óptico. Fig. 91. Heterodinium blackmani, vista ventral. Fig. 92. Karenia asteriochroma, vista ventral. Fig. 93. K. brevisulcata, vista dorsal. Fig. 94. Nematodinium torpedo. Fig. 95. Torodinium teredo. Fig. 96. Neoceratium biceps, vista ventral. Fig. 97. Polykrikos kofoidii, vista dorsal. Fig. 98. Takayama cf. cladochroma, vista ventral. Fig. 99. Pyrocystis gerbaulti. Fig. 100. N. vultur f. japonicum, vista dorsal. Fig. 101. Scaphodinium miriabile. Fig. 102. Warnowia juno, vista ventral. Fig. 103. W. maxima, envoltura mucilaginosa. Fig. 104. C. carriense var. volans, vista ventral. Escalas: Fig. $98=10 \mu \mathrm{m}$; Figs. 92-95, 97, $102=20 \mu \mathrm{m}$; Figs. 91, 101, $103=50 \mu \mathrm{m}$; Figs. 96, 99-100, $104=100 \mu \mathrm{m}$. 
Meave-del Castillo et al.: Ficoflora planctónica de la Bahía de Acapulco, México
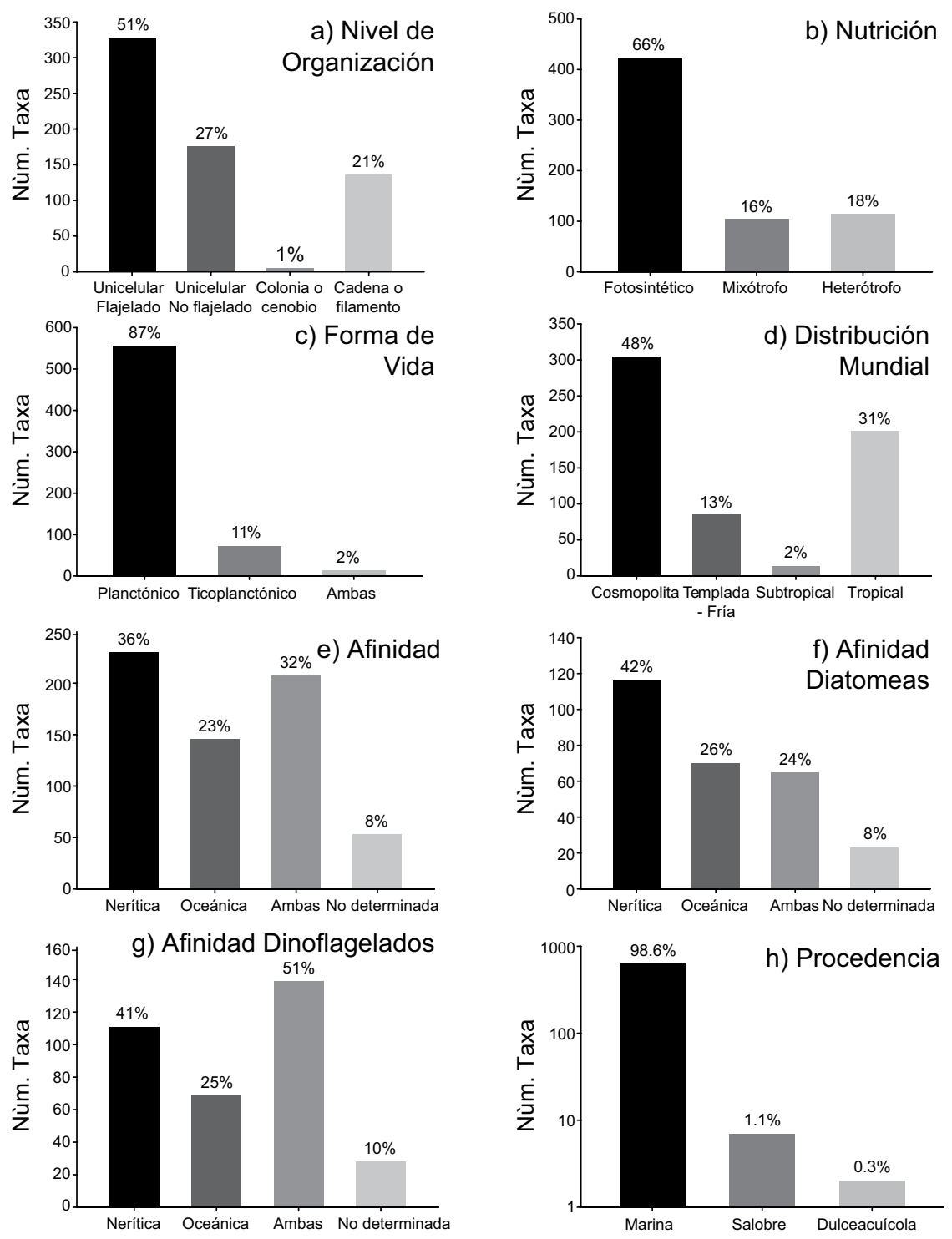

Figs. 105a-h. Figs. 105 a-e, h. Porcentaje de las características del total de taxa encontrados en la Bahía de Acapulco. a) Nivel de organización; b) Tipo de Nutrición; c) Forma de vida; d) Distribución mundial, para el cómputo de los taxa cosmopolitas se consideraron todos los reportados en la literatura con esa categoría, o bien los registrados en una amplitud latitudinal: tropical-templada y tropical-fría; e) Afinidad (nerítica u oceánica) de todos los taxa; f) Porcentaje de la afinidad (nerítica u oceánica) de los taxa del grupo de diatomeas (Bacillariophyta); g) Porcentaje de la afinidad (nerítica u oceánica) de los taxa del grupo de dinoflagelados (Dinophyta); h) Procedencia (marina, salobre o dulceacuícola). 
cytis sp. (Fig. 39-41), con especies de dinoflagelados Dinophysiales (Ornithocercus spp. (Figs. 39-40) e Histioneis sp. (Fig. 41) y diatomeas, tales como Planktoniella sol y Helicotheca tamesis; al igual que la presencia de Richelia intracellularis dentro de Rhizosolenia spp. También se observó al ciliado Myrionecta rubra Jankowski, en densidades de hasta 57 x $10^{3} \mathrm{Céls}^{\mathrm{L}} \mathrm{L}^{-1}$ (enero/2011) en la localidad denominada "La Marina", conteniendo endosimbiontes fotosintéticos (criptofitas).

Otra asociación interesante fue el hallazgo de un gusano platelminto, acelomado, de la Clase Turbellaria, del grupo de los Convolútidos, conteniendo en su interior gran cantidad de endosimbiontes autótrofos de color marrón (Figs. 106-108), con morfología semejante a dinoflagelados desnudos del género Amphidinium (Fig. 109), hecho que será importante verificar en un futuro con estudios de microscopía electrónica de transmisión. La asociación de Convolútidos con dinoflagelados guían al género Amphicolops (Taylor, 1971; Mendes-Lopes \& Silveira, 1994), que actualmente ha sido reubicado en Heterochaerus, por presentar una estructura sexual masculina peculiar, llamada bolsa seminal celular (Achatz et al., 2009). Este platelminto "autótrofo", de 305-421 $\mu \mathrm{m}$ de longitud, se encontró en la columna de agua, con abundancias relativas de hasta $38 \%$, principalmente en la localidad denominada " $\mathrm{La}$ Naval", durante gran parte del bienio 2009-2010, donde también fue común hallar representantes de Euglenophyta. En la literatura se señala que cuando los Convolutidos son jóvenes se alimentan de materia orgánica, como lo hacen otras planarias de vida libre, y que justo en estos estadios juveniles es cuando consumen las algas sin digerirlas, hasta obtener una cantidad suficiente para su mantenimiento posterior, ya que en los estadios adultos pierden su intestino y dependen completamente de las microalgas para su nutrición, convirtiéndose en una simbiosis mutualista.

También se pudieron reconocer relaciones de parasitismo sobre todo en la época de secas, tales como: Chytriodinium affine en huevos de copépodos (Fig. 71) y Oodinium sp. sobre salpas de Oikopleura sp. (Fig. 110); y otros dinoflagelados endoparásitos de copépodos y tintínidos. Adicionalmente se observaron pequeñas diatomeas pennadas sobre las quetas de Chaetoceros spp. Todo lo anterior refleja la existencia de una comunidad fitoplanctónica compleja en la Bahía de Acapulco.

Otra relación simbiótica que llamó la atención y que puede ayudar a entender el estado trófico de la bahía de Acapulco a lo largo del año, fue la del consorcio Leptocilindrus mediterraneus (¿diatomea?)-Solenicola setigera (protozoario; Figs. 111-113). Dado que a la fecha no se han podido observar frústulas de la diatomea con cloroplastos (Hasle, 1975; Taylor, 1982; Gómez, 2007), e incluso existe la duda de si ellas presentan o no citoplasma, porque los únicos organelos observados en su interior han sido mitocondrias (Buck \& Bentham, 1998), se menciona que la "diatomea" 

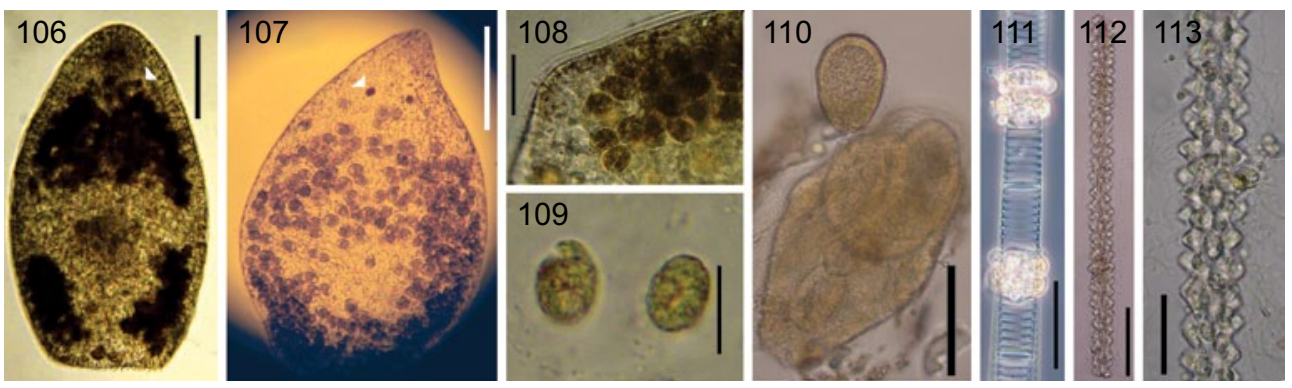

Figs. 106-113. Simbiontes y parásitos. Figs. 106-109. Platelminto del grupo Convolutidae con endosimbiontes del género Amphidinium. Figs. 106-107. Organismos completos conteniendo numerosos endosimbiontes de color marrón, la punta de flecha indica los ocelos. Fig. 108. Detalle de la pared del platelminto. Fig. 109. Amphidinium sp., endosimbionte excretado por el platelminto. Fig. 110. Oodinium sp., sobre salpa. Figs. 111-113. Consorcio Leptocylindrus mediterraneus-Solenicola setigera. Fotografías tomadas al microscopio óptico. Escalas: Fig. $109=10 \mu \mathrm{m}$; Figs. 108, 110, $113=20 \mu \mathrm{m}$; Figs. 111-112 = $50 \mu \mathrm{m}$; Figs. 106-107= $100 \mu \mathrm{m}$.

no existe como tal, sino que la "frústula" es parte de una estructura del protozoario que sirve como soporte para el crecimiento masivo del mismo (Gómez, 2007). Se ha mencionado que este consorcio es más común en aguas ricas en nutrientes (Gómez, 2007) usando como explicación que $S$. setigera se nutre de picoplancton, del cual algunas especies son más abundantes en aguas eutróficas (Blanchot \& Rodier, 1996). Sin embargo, durante el mes de enero de 2011, época en la que la abundancia del consorcio Solenicola fue mayor, y además se observó el estadio de "frústulas" cubiertas completamente de protozoarios con flagelos (Figs. 112-113), a los cuales se les habían pegado células picoplanctónicas, los nutrientes tuvieron concentraciones bajas de fosfatos y nitratos respectivamente, pero en cambio ligeramente altas para el amonio (Fig. 2c). Lo anterior parece apoyar más la idea de Buck y Bentham (1998), quienes señalan que este consorcio (L. mediterraneus-S. setigera), se ve beneficiado cuando a su vez se favorecen especies fijadoras de nitrógeno, tales como Synechococcus sp., debido a la limitación de nitratos en la columna de agua. Dicha teoría fue validada en Acapulco, al observar la presencia de feosomas de cianofitas en los velos cingulares de dinoflagelados Dinophysiales y diversas diatomeas, como fue mencionado más arriba, al igual que la presencia de Richelia intracellularis dentro de Rhizosolenia spp.

\section{Esfuerzo de muestreo}

Analizando la riqueza de algas reconocidas en la Bahía de Acapulco, colectados con los distintos métodos de muestreo (Apéndice 1), se puede observar que 
del total de taxa (641) registrados, 197 fueron observados únicamente en muestras de botella (30.7\%), 129 (20.1\%) en las de red, y los siguientes 12 (1.9\%), solo mediante la observación de muestras vivas: Cochlodinium convolutum, C. fulvescens, Gymnodinium ravenescens, G. rhomboides, G. sphaericum, G. translucens, Gyrodinium foliaceum, Gyrodinium cf. ochraceum, G. pepo, Takayama cf. cladochroma, Warnowia violescens y Fibrocapsa japonica. Los taxa restantes (304, equivalentes a 47.4\%) se encontraron en muestras colectadas con dos o tres de los métodos utilizados (Fig. 114, Apéndice 1).

En la Fig. 115a se puede observar que la curva de acumulación de especies para las muestras de botella presentó un nivel asintótico adecuado (más evidente después de la permutación en la secuencia de muestras), el cual alcanzó el valor de riqueza máxima estimada $\left(\mathrm{S}_{\max }=618\right)$. Para las de red $(20$ y $54 \mu \mathrm{m}$ de abertura de malla; Fig. 115b), tanto la riqueza observada para cada muestra, como sus correspondientes valores después de la permutación, señalaron un comportamiento asintótico similar, pero en este caso, sin alcanzar el valor de riqueza máxima estimada $\left(\mathrm{S}_{\max }=430\right)$. De acuerdo con el comportamiento de las curvas de acumulación de especies y los valores de $\mathrm{S}_{\text {max }}$ obtenidos en ambos tipos de muestreo (botella y red), se puede considerar que la riqueza fitoplanctónica que se ha documentado al presente, seguramente corresponde a una estimación satisfactoria de la diversidad potencial de la Bahía de Acapulco, sobre todo para la información obtenida de capturas de botella. La diferencia encontrada para ambos tipos de muestra (en riqueza observada y $\mathrm{S}_{\max }$ ) se puede

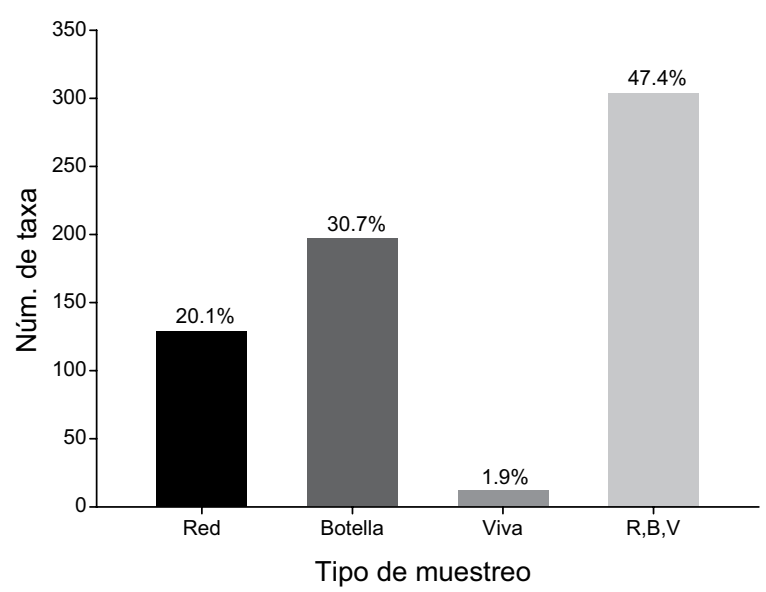

Fig. 114. Distribución de taxa encontrados por tipo de muestreo. 
explicar en parte por aspectos metodológicos, debido a que con la botella se colectan varios sitios (diferentes niveles de profundidad) y por ello puede estar mejor representada la ficoflora de toda la columna de agua. Obviamente el esfuerzo de captura fue mayor en las muestras de botella. No obstante lo anterior, otra explicación que podría darle valor a los datos de riqueza específica que arrojaron las muestras de red, podría consistir en el hecho de que, mientras que las colectas de botella correspondieron a un estudio continuado a través de un ciclo anual, y por tanto sus resultados podrían representar el censo de una comunidad rica, pero más homogénea; las colectas con red, hechas de manera esporádica en un periodo largo, podrían reflejar la presencia de especies pertenecientes a distintas comunidades fitoplanctónicas, presentes en la Bahía de Acapulco en tiempos distintos, registradas en diversos momentos, a través de una década desde el año 2000, por lo cual reflejarían modificaciones en la composición no solo temporales a través de ciclos anuales o bianuales, sino también a más largo plazo y que podrían estar influenciados por cambios producidos por fenómenos globales, tales como El Niño (ENSO, El Niño South Oscillation).

Del total de las muestras analizadas, únicamente 25 taxa tuvieron valores de frecuencia mayores de 50\% (Apéndice 1), y por ello pueden ser calificadas como
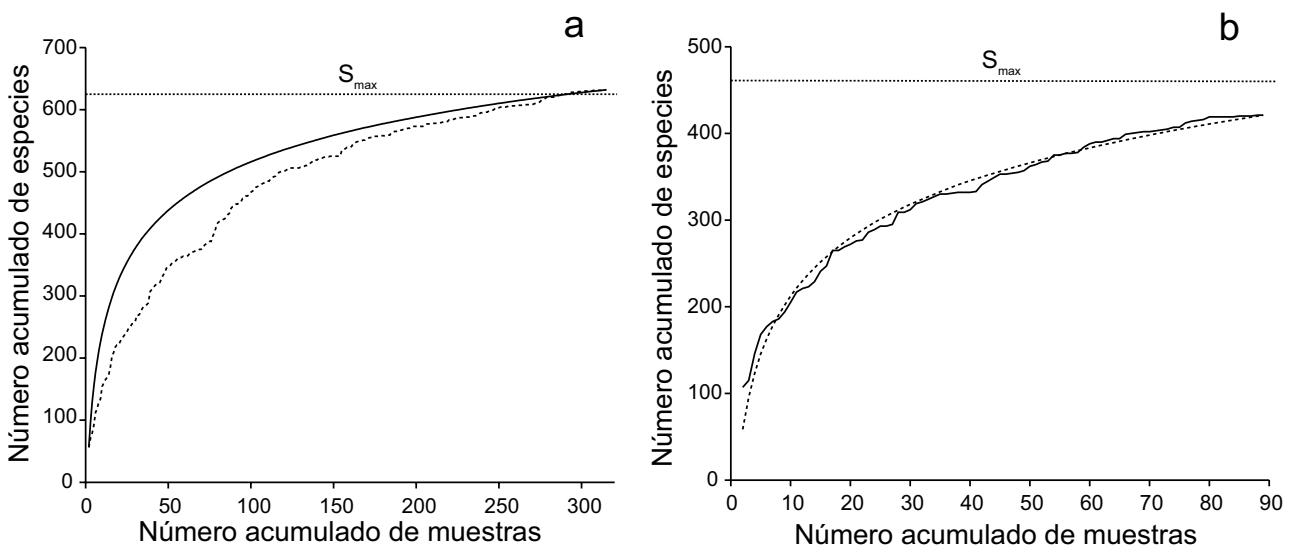

Figs. 115a-b. a) Curva de acumulación de especies fitoplanctónicas para las muestras de botella. Riqueza de especies observada por muestra (línea discontinua) y riqueza de especies por muestra después de 100 permutaciones (línea continua). También se señala el nivel asintótico definido por la constante Smax (línea punteada). b) Curva de acumulación de especies fitoplanctónicas para las muestras de red. Riqueza de especies observada por muestra (línea discontinua) y riqueza de especies por muestra después de 100 permutaciones (línea continua). También se señala el nivel asintótico definido por la constante Smax (línea punteada). 
típicas de la bahía de Acapulco. Corresponden a 14 diatomeas: Chaetoceros curvisetus (Fig. 116), C. decipiens (Fig. 117), C. laciniosus (Fig. 118), Cylindrotheca closterium (Fig. 119), Dactyliosolen fragilissimus (Fig. 120), Ditylum brightwellii (Fig. 121), Guinardia striata (Fig. 122), Helicotheca tamesis (Fig. 123), Hemiaulus hauckii (Fig. 124), Leptocylindrus danicus (Fig. 125), Pseudo-nitzschia pseudodelicatissima (Fig. 126), Rhizosolenia pungens (Fig. 127), Thalassionema frauenfeldii (Fig. 128) y T. nitzschioides (Fig. 129); y once dinoflagelados: Akashiwo sanguinea (Fig. 130), Diplopsalopsis bomba (Fig. 131), Dinophysis caudata (Fig. 132), Gyrodinium acutum (Fig. 82), G. fusus (Fig. 133), Neoceratium furca (Figs. 134-135), Neoceratium (Ceratium) furca var. eugrammum (Figs. 136 y 138), N. fusus (Fig. 137), Prorocentrum gracile (Figs. 139a y 140), P. micans (Figs. 139b y 141) y Protoperidinium divergens (Fig. 142). Entre la lista de estas especies, siete son consideradas como tóxicas o nocivas (Apéndice 1).

Abundancia de las especies

En el Apéndice 1 se presentan los máximos y mínimos de abundancia para cada uno de los 513 taxa observados en muestras de botella; con un mínimo de 10 Céls. $\mathrm{L}^{-1}$ y un máximo de 773,554 Céls. $\mathrm{L}^{-1}$ para el dinoflagelado tóxico Pyrodinium bahamense var. compressum. Se observaron valores mayores a $100 \times 10^{3} \mathrm{Céls} \mathrm{L}^{-1}$ en 17 taxa de Bacillariophyta de la clase Coscinodiscophyceae, y en otras tres de la clase

Figs. 116-142. Taxa típicos en la Bahía deAcapulco, ejemplares vistos bajo el microscopioóptico, excepto la figuras $124,131,132,134,136,137,138,140,141$ y 142 tomadas con microscopio electrónico de barrido. Fig. 116. Chaetoceros curvisetus, cadena en vista cingular. Fig. 117. C. decipiens, cadena en vista conectiva. Fig. 118. C. laciniosus, cadena en vista conectiva. Fig. 119. Cylindrotheca closterium, vista valvar. Fig. 120. Dactyliosolen phuketensis, cadena en vista conectiva. Fig. 121. Ditylum brightwelli, célula en vista conectiva. Fig. 122. Guinardia striata, vista conectiva. Fig. 123. Helicotheca tamesis, vista conectiva. Fig. 124. Hemiaulus hauckii, vista conectiva. Fig. 125. Leptocylindrus danicus, vista conectiva. Fig. 126. Pseudonitzschia pseudodelicatissima, vista valvar. Fig. 127. Rhizosolenia pungens, vista conectiva. Fig. 128. Thalassionema frauenfeldii, vista valvar. Fig. 129. Thalassionema nitzschioides, vista conectiva. Fig. 130. Akashiwo sanguinea, vista ventral. Fig. 131. Diplopsalopsis bomba, vista apical. Fig. 132. Dinophysis caudata, vista lateral izquierda. Fig. 133. Gyrodinium fusus, vista dorsal. Figs. 134-135. Neoceratium furca, vista ventral. Figs. 136, 138. C. furca var. eugrammum, vista ventral. Fig. 137. N. fusus, vista ventral. Figs. 139a, 140. Prorocentrum gracile, vista lateral derecha. Figs. 139b, 141. Prorocentrum micans, vista lateral derecha. Fig. 142. Protoperidinium divergens, vista ventral. Escalas: Fig. $124=5 \mu \mathrm{m}$; Figs. 130-132, 138, 140-141 = $10 \mu \mathrm{m}$; Figs. 119-120, 122, 126-129, 133, 134-137, 139a-b, 141-142 = $20 \mu \mathrm{m}$; Figs. $116-118,121,123,125,137=50 \mu \mathrm{m}$. 
Meave-del Castillo et al.: Ficoflora planctónica de la Bahía de Acapulco, México
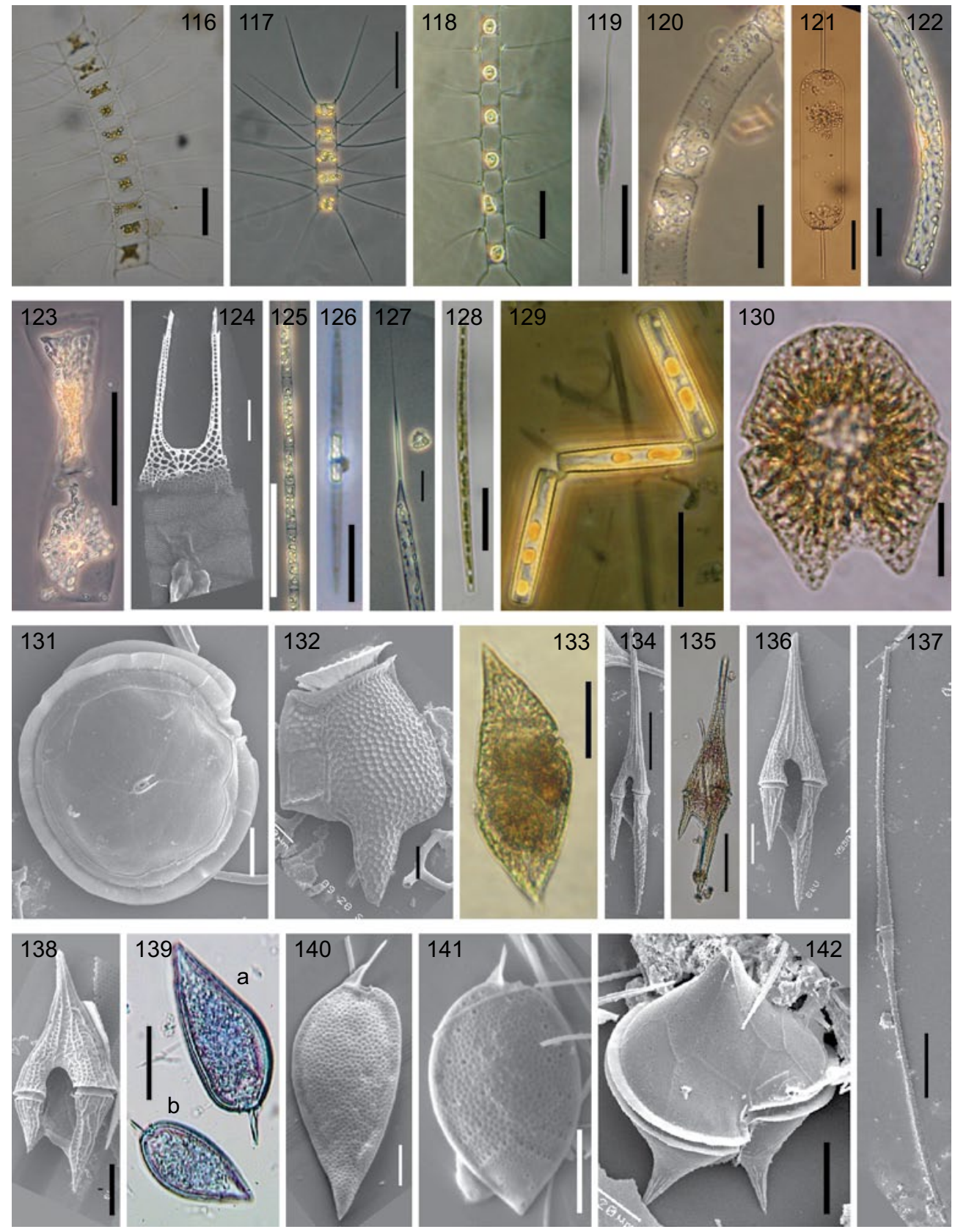

Pie de figura en página opuesta. 
Bacillariophycea del género Psudo-nitzschia (Apéndice 1). Entre los dinoflagelados, solo cinco presentaron valores de abundancia mayores a $100 \times 10^{3} \mathrm{Céls} \mathrm{L}^{-1}$, correspondientes a dos especies desnudas (Gymnodinium catenatum y G. attenuatum) y tres tecadas (Scrippsiella trochoidea, Scrippsiella sp. 2 y Pyrodinium bahamense var. compressum). De los grupos algales restantes, solo Phaeocystis sp. y Trychodesmium erythraeum presentaron valores superiores a las $100 \times 10^{3} \mathrm{Céls} \mathrm{L}^{-1}$. Por otra parte, $42 \%$ de los taxa (216) registró valores inferiores a $1 \times 10^{3} \mathrm{Céls} \mathrm{L}^{-1}$, y en los correspondientes al 40\% (203) restante, tuvieron valores máximos entre 1-10 x $10^{3}$ Céls. $\mathrm{L}^{-1}$ (Apéndice 1). Por lo que en términos generales, la mayoría de los taxa fitoplanctónicos (82\%) en la bahía de Acapulco, se encuentran distribuidos de manera proporcional con bajas concentraciones en la columna de agua $\left(<10 \times 10^{3}\right.$ Céls. $\left.\mathrm{L}^{-1}\right)$.

\section{Abundancia relativa}

A partir de 22,080 registros de abundancia relativa de los taxa, obtenidos del conteo de 88 muestras de red y 364 de botella, se mostró que la mayoría caen en la clasificación de raros (96\%), seguido de los escasos (3.25\%), los comunes $(0.66 \%)$ y solo $0.1 \%$ de los abundantes ( 23 taxa); estos últimos son los que pueden ser reconocidos como FAN. Lo anterior indica que en general, no hay dominancia de especies en las muestras y por tanto en la bahía. Estos datos nos muestran que a pesar de que en la región de estudio ocurren FAN, que incluso han causado afectaciones a la salud humana y a la economía, dichos eventos son más bien raros y esporádicos.

Especies formadoras de FAN en Acapulco

En el Cuadro 4 se presenta el listado de los florecimientos algales registrados en la Bahía de Acapulco a lo largo del periodo de estudio, señalando su fecha de ocurrencia y la densidad en células por litro (en el caso de muestra de botella), así como la abundancia relativa tanto en el caso de capturas de red como de botella. Con las muestras analizadas, se pudieron registrar 12 eventos FAN en el transcurso de 11 años, involucrando a 23 taxa, repartidos homogéneamente entre diatomeas y dinoflagelados, $4.0 \%$ reportados como tóxicos o nocivos. Por otra parte, de los 641 taxa registrados para la región de estudio, 54 (8.4\%), principalmente dinoflagelados, están señalados en la literatura como tóxicos o nocivos, por lo cual por ahora únicamente pueden ser considerados potencialmente nocivos para la bahía de Acapulco.

Entre los florecimientos registrados desde el año 2000, los siguientes diez taxa se consideran tóxicos o nocivos: Chaetoceros curvisetus (Fig. 116), Leptocylindrus minimus (Fig. 146), Pseudo-nitzshia cf. pseudodelicatissima (Fig. 126), Akashiwo sanguinea (Fig. 130), Cochlodinium polykrikoides (Fig. 150), Gymnodinium 
Cuadro 4. Especies que formaron florecimientos del 2000 al 2010. Se reporta la densidad en células por litro (Céls. $\mathrm{L}^{-1}$ ) y abundancia relativa en porcentaje (A.R.), así como la localidad, profundidad y fecha en la que fueron colectadas, además del número de figura que la ilustra. En negritas se señalan las especies reportadas como tóxicas o nocivas.

\begin{tabular}{|c|c|c|c|c|c|c|}
\hline Taxon & Céls. L ${ }^{-1}$ & A.R. & Localidad & Prof. & Fecha & Fig. \\
\hline \multirow[t]{3}{*}{ Chaetoceros curvisetus } & - & $82 \%$ & Centro & & $11 / 2002$ & 116 \\
\hline & 163600 & $80 \%$ & Centro & $1 \mathrm{~m}$ & $07 / 2010$ & \\
\hline & 660725 & $83 \%$ & Centro & $20 \mathrm{~m}$ & $09 / 2010$ & \\
\hline Chaetoceros laciniosus & 276449 & $80 \%$ & Centro & $20 \mathrm{~m}$ & $01 / 2011$ & 118 \\
\hline Chaetoceros radicans & 266675 & $80 \%$ & Muelle & $5 \mathrm{~m}$ & $09 / 2010$ & 143 \\
\hline Chaetoceros tortissimus & 364678 & $81 \%$ & Bocana & $3 \mathrm{~m}$ & $01 / 2011$ & 144 \\
\hline \multirow[t]{2}{*}{ Climacodium frauenfeldianum } & - & $86 \%$ & Bocana & & $05 / 2000$ & 145 \\
\hline & - & $82 \%$ & Muelle & & $10 / 2000$ & \\
\hline \multirow[t]{2}{*}{ Leptocylindrus danicus } & - & $91 \%$ & Bocana & & $05 / 2003$ & 125 \\
\hline & 145492 & $80 \%$ & Centro & $1 \mathrm{~m}$ & $07 / 2010$ & \\
\hline Leptocylindrus minimus & 287439 & $85 \%$ & Bocana & $5 \mathrm{~m}$ & $03 / 2010$ & 146 \\
\hline $\begin{array}{l}\text { Pseudo-nitzschia cf. } \\
\text { pseudodelicatissima }\end{array}$ & 306401 & $82 \%$ & Muelle & $3 \mathrm{~m}$ & $11 / 2010$ & 126 \\
\hline $\begin{array}{l}\text { Pseudo-nitzschia } \mathrm{cf} . \\
\text { subfraudulenta }\end{array}$ & - & $82 \%$ & Muelle & & $11 / 2002$ & 147 \\
\hline $\begin{array}{l}\text { Skeletonema cf. } \\
\text { pseudocostatum }\end{array}$ & 568127 & $84 \%$ & Sinfonía & $5 \mathrm{~m}$ & $07 / 2008$ & 148 \\
\hline \multirow[t]{2}{*}{ Skeletonema sp. 1} & - & $83 \%$ & costera & & $11 / 2002$ & 149 \\
\hline & 268110 & $80 \%$ & Sinfonía & $3 \mathrm{~m}$ & $11 / 2010$ & \\
\hline Thalassionema nitzschioides & 66417 & $81 \%$ & Sinfonía & $3 \mathrm{~m}$ & $07 / 2010$ & 129 \\
\hline Akashiwo sanguinea & - & $80 \%$ & Centro & & $05 / 2003$ & 130 \\
\hline Cochlodinium polykrikoides & - & $82 \%$ & P. Marqués & & $05 / 2007$ & 150 \\
\hline Gymnodinium catenatum & - & $88 \%$ & Naval & & $05 / 2003$ & 151 \\
\hline Neoceratium balechii & - & $80 \%$ & Centro & & $05 / 2003$ & 152 \\
\hline $\begin{array}{l}\text { Neoceratium (Ceratium) } \\
\text { balechii var. longum }\end{array}$ & - & $81 \%$ & Centro & & $05 / 2000$ & 153 \\
\hline Neoceratium furca & - & $85 \%$ & Muelle & & $05 / 2000$ & $134-135$ \\
\hline Prorocentrum gracile & - & $80 \%$ & Bocana & & $10 / 2009$ & $139 a, 140$ \\
\hline Prorocentrum micans & - & $83 \%$ & Centro & & $10 / 2009$ & $139 b, 141$ \\
\hline Protoperidinium divergens & - & $80 \%$ & Centro & & $06 / 2010$ & 142 \\
\hline $\begin{array}{l}\text { Pyrodinium bahamense var. } \\
\text { compressum }\end{array}$ & 773544 & $86 \%$ & Centro & $3 \mathrm{~m}$ & $07 / 2010$ & $154-155$ \\
\hline Scrippsiella trochoidea & 111123 & $81 \%$ & Sinfonía & $1 \mathrm{~m}$ & $10 / 2009$ & 156 \\
\hline
\end{tabular}



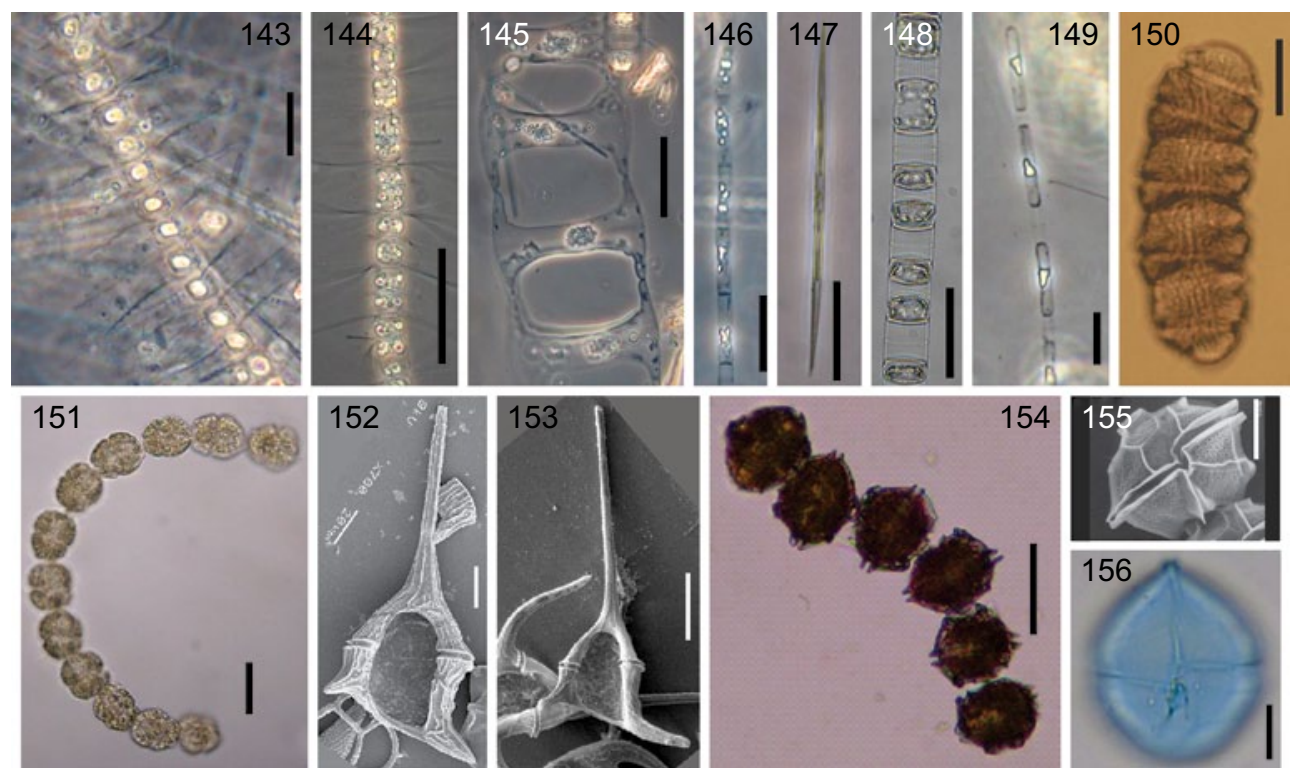

Figs. 143-156. Taxa formadores de FAN, ejemplares vistos bajo el microscopio óptico, excepto la figuras 152, 153 y 155 con microscopio electrónico de barrido. Fig. 143. Chaetoceros radicans, cadena en vista conectiva. Fig. 144. C. tortissimus, cadena en vista conectiva. Fig. 145. Climacodium frauenfeldianum, cadena en vista conectiva. Fig. 146. Leptocylindrus minimus, vista conectiva. Fig. 147. Pseudo-nitzschia subfraudulenta, vista valvar. Fig. 148. Skeletonema cf. pseudocostatum, cadena en vista conectiva. Fig. 149. Skeletonema sp. 1, cadena en vista conectiva. Fig. 150. Cochlodinium polykrikoides, vista dorsal. Fig. 151. Gymnodinium catenatum, células en cadena. Fig. 152. Neoceratium balechii, vista ventral. Fig. 153. C. balechii f. longum, vista ventral. Figs. 154-155. Pyrodinium bahamense var. compressum: Fig. 154. Células en cadena; Fig. 155. Célula en vista lateral derecha. Fig. 156. Scrippsiella trochoidea, vista ventral. Escalas: Figs. 150, $156=10 \mu \mathrm{m}$; Figs. 143, 146, 148 $149,151-152=20 \mu \mathrm{m}$; Figs. 144-145, 147, 153-155= $50 \mu \mathrm{m}$.

catenatum (Fig. 151), Neoceratium furca (Figs. 134-135), Prorocentrum micans (Figs. 140-141) Pyrodinium bahamense var. compressum (Figs. 154-155) y Scripsiella trochoidea (Fig. 156). Dos de ellos: Gymnodinium catenatum y Pyrodinium bahamense var. compressum son productores de saxitoxinas y causan alta toxicidad en diversas partes del mundo. Sin embargo, P. bahamense var. compressum ha sido el que en Acapulco, ha ocasionado los mayores problemas de salud pública. Durante un FAN ocurrido a finales de 1995 e inicios de 1996, en las costas de los estados de Guerrero y Michoacán, incluyendo la bahía de Acapulco, se registraron valores de saxitoxinas en el bivalvo Ostrea iridiscens, de hasta $6,337 \mathrm{mg}$ STXeq $100 \mathrm{~g}^{-1}$, causan- 
do varias personas intoxicadas y seis muertes (Orellana et al., 1998). Asimismo de agosto de 2001 a febrero de 2002, P. bahamense produjo otro FAN con densidades de hasta $3 \times 10^{6}$ Céls. $\mathrm{L}^{-1}$, junto con $G$. catenatum y Prorocentrum sp., causando concentraciones de saxitoxinas en bivalvos de hasta 7,309 mg STXeq $100 \mathrm{~g}^{-1}$, provocando el envenenamiento de más de 100 personas, nueves fallecimientos, 180 días de veda y $48 \mathrm{t}$ de peces muertos (Ramírez-Camarena et al., 2004). Los últimos FAN de este dinoflagelado registrados en la bahía, ocurrieron durante el desarrollo del presente estudio, en el periodo comprendido entre julio/2010 a enero/2011. El primero acontecido en julio, fue intenso con concentraciones de hasta $773.5 \times 10^{3}$ Céls. L-1 en el centro de la bahía, a $3 \mathrm{~m}$ de profundidad; con valores máximos de toxicidad de $2,092 \mu \mathrm{gSXTeq} 100 \mathrm{~g}^{-1}$ de carne con bioensayo con ratón registrados el día 8 de julio de 2010 (Anónimo, 2010a) y de $894.57 \mu \mathrm{gSXTeq} 100 \mathrm{~g}^{-1}$ de carne por HPLC, el 24 de julio de 2010 (Gárate-Lizárraga et al., 2012). En septiembre las concentra-

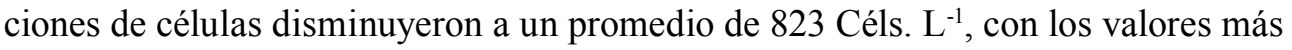
altos encontrados a $10 \mathrm{~m}$ de profundidad; aunque se continuó registrando valores de toxicidad todavía altos, de $100 \mu \mathrm{gSXTeq} 100 \mathrm{~g}^{-1}$ de carne con bioensayo con ratón, el 4 de octubre de 2010 (Anónimo, 2010a). En noviembre se presentó nuevamente un incremento de la especie con densidades de 12.3 y 16.5 x $10^{3}$ Céls. $\mathrm{L}^{-1}$, a 5 y $10 \mathrm{~m}$ en las localidades Sinfonía y Pta. Bruja, respectivamente, y valores de saxitoxinas de hasta $739 \mu \mathrm{gSXTeq} 100 \mathrm{~g}^{-1}$ de carne con bioensayo con ratón, el 12 de noviembre de 2010 (Anónimo, 2010a). Este incremento es considerado como un nuevo evento que se gestó en el exterior de la bahía, toda vez que coincidió con un FAN de mayor magnitud, ocurrido a lo largo de la costa grande del estado de Guerrero, durante diciembre de 2010 (Gárate-Lizárraga et al., 2011), el cual estuvo conformado por cuatro especies de dinoflagelados, dando valores de toxicidad de hasta $536 \mu \mathrm{gSXTeq}$ $100 \mathrm{~g}^{-1}$ de carne con bioensayo con ratón. En la región de estudio, P. bahamense var. compressum, continuó presentándose hasta enero de 2011, en las estaciones ubicadas al E de la bahía, aunque ya no se reportaron valores de saxitoxinas por arriba de los $80 \mu \mathrm{gSXTeq} 100 \mathrm{~g}^{-1}$ de carne. Durante todo el periodo en que $P$. bahamense var. compressum estuvo presente en la Bahía de Acapulco (julio 2010 a enero 2011), la

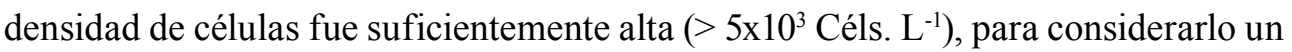
FAN, y las concentraciones de toxinas alcanzadas, guiaron a la veda en la extracción de moluscos hasta el 12 de noviembre de 2010.

Otro dinoflagelado tóxico productor de saxitoxinas es Gymnodinium catenatum, que estuvo presente en Acapulco durante todos los muestreos con abundancias que variaron de 20 a $188.7 \times 10^{3} \mathrm{Céls} \mathrm{L}^{-1}$. En la época de secas (octubre de $2010 \mathrm{a}$ mayo de 2010), los mencionados valores fueron menores a $4 \times 10^{3} \mathrm{Céls} \mathrm{L}^{-1}$, pero a 
principios de la época de lluvias (julio) incrementó su abundancia llegando casi a $10 \times 10^{3}$ Céls. $\mathrm{L}^{-1}$, coincidiendo el comportamiento de su profusión con el FAN de Pyrodinium bahamense var. compressum. En septiembre sus abundancias decayeron hasta un promedio de $129 \mathrm{Céls}^{\mathrm{L}} \mathrm{L}^{-1}$, al igual que Pyrodinium bahamnese; repuntando en el mes de noviembre $\left(188.7 \times 10^{3} \mathrm{Céls} \mathrm{L}^{-1}\right)$, por lo que seguramente esta especie contribuyó también en las concentraciones de saxotoxinas alcanzadas en los moluscos bivalvos (392-739 $\mu \mathrm{gSTXeq} / 100 \mathrm{~g}^{-1}$, el día 12 de noviembre de 2010; Anónimo, 2010a). En enero de 2011 los valores bajaron nuevamente aunque en algunos sitios fueron mayores de $5 \times 10^{3}$ Céls. $\mathrm{L}^{-1}$, con las cantidades más altas entre los 3 y 5 metros de profundidad.

Grupos importantes adicionales registrados en Acapulco y también considerados organismos tóxicos, correspondieron a los dinoflagelados del género Karenia, de los cuales se encontraron ocho taxa en la Bahía de Acapulco: Karenia asteriochroma (Fig. 92), K. bicuneiformis (Fig. 157), K. cf. brevis (Fig. 60), K. brevisulcata (Fig. 93), K. mikimotoi (Fig. 158), K. papillonacea (Figs. 159-160), K. selliformis (Fig. 161) y Karenia sp. 1 (Fig. 162). Las representantes más comunes en Acapul-
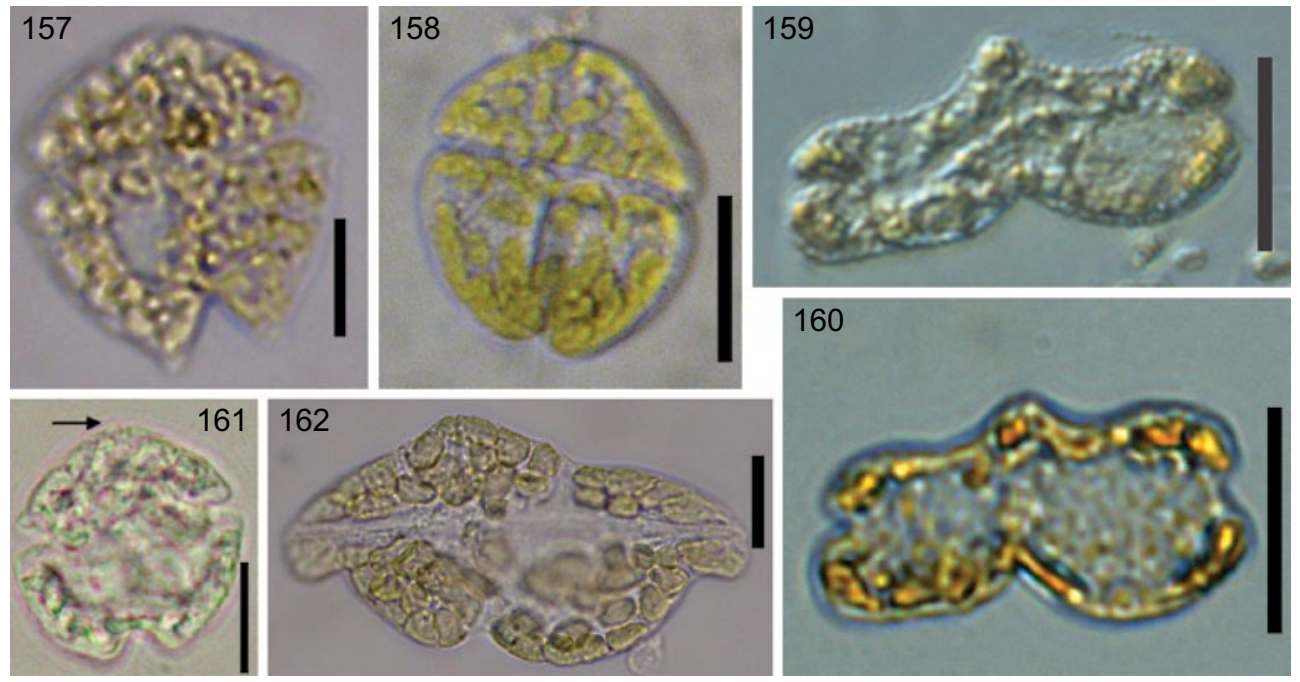

Figs. 157-162. Taxa del género Karenia, ejemplares vistos bajo el microscopio óptico. Fig. 157. Karenia bicuneiformis, vista dorsal. Fig. 158. K. mikimotoi, vista ventral. Figs. 159-160. K. papillonacea, vista ventral. Fig. 161. K. selliformis, vista dorsal, la flecha señala la carena. Fig. 162. Karenia sp., vista ventral. Escalas: Figs. 157, $161=10 \mu \mathrm{m}$; Figs. 158-160, $162=$ $20 \mu \mathrm{m}$. 
co fueron: K. mikimotoi y K. selliformis, alcanzando densidades de hasta $1.8 \times 10^{3}$ Céls. $\mathrm{L}^{-1}$ en agua superficial (1-3m). Otra de las especies interesantes, que constituyó además un nuevo registro para el PM, fue $K$. asteriochroma. Estimando el valor de

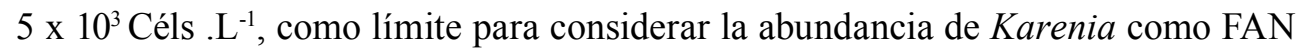
(Lansberg \& Steidinger, 1998), y sabiendo que K. breve, K. mikimotoi y K. selliformis son ictiotóxicas (productoras de hemolisinas y gimnodiminas), se puede afirmar que tales especies representan un riesgo para la salud en la Bahía de Acapulco, sobre todo porque se les ha encontrado junto con Gymnodinium catenatum.

\section{CONCLUSIONES}

Como conclusión cabe aseverar que la ficoflora planctónica y ticoplanctónica de Acapulco es rica (641 taxa) y compleja, siendo importantes los dinoflagelados, tanto tecados como desnudos, así como las diatomeas. La alta biodiversidad fitoplanctónica encontrada fue todavía más sorprendente, cuando al compararla con otras seis bahías ubicadas en el PM, resultó ser la de mayor riqueza e incluso semejante a la reportada en los golfos de California y Tehuantepec, regiones más extensas y dinámicas desde el punto de vista oceanográfico.

Es interesante que solo 27 taxa mostraron tener una frecuencia de aparición mayor de 50\%, lo que evidencia la sucesión de distintas comunidades fitoplanctónicas a lo largo de los 10 años de periodo de estudio. El hecho de que la mayoría de las especies sean escasas o raras, muestra que la alta riqueza de algas fitoplanctónicas en Acapulco, es resultado en parte, del hecho de que las especies dominantes sean muy pocas y con baja abundancia, lo que permite la expresión de un gran número de taxa en la bahía. Dicho hallazgo parece no concordar con la idea de que Acapulco sea un ambiente altamente eutrofizado por efectos antropógenas. Otra diferencia importante del área de estudio con respecto a otras regiones del PM es su mayor proporción de taxa de Dinophyta en comparación con las diatomeas (los grupos mayoritarios en el fitoplancton marino), lo cual además también fue registrado por Rojas-Herrera et al. (2012).

Este estudio contribuyó con 38 nuevos registros para el Pacífico mexicano particularmente (36) dinoflagelados, de los cuales la mayoría son especies desnudas o atecadas, probablemente como resultado de la pronta observación de las muestras fijadas y de la observación de muestras in vivo.

Con base en el análisis del esfuerzo de muestreo realizado, se puede concluir que la captura con botella permitió obtener una representación fiel de la riqueza de 
especies del sistema y afirmar que básicamente ya se tiene un conocimiento bastante completo de la riqueza fitoplanctónica de la bahía, sin embargo todavía es necesario enfocar un estudio específico sobre fitoflagelados y picoplancton para completar el listado. Por otra parte, el alto número de especies fitoplanctónicas, registradas únicamente a través de las colectas con red, evidenció que dadas las variaciones de las condiciones ambientales que pueden ocurrir en la bahía, para llegar a conocer cabalmente a las especies que habitan en Acapulco, además de realizar trabajo intensivo durante ciclos anuales o bimestrales, son necesarios los estudios a largo plazo, como los que se han realizado a través de más de 10 años, por personal del Laboratorio de Fitoplancton Marino y Salobre de la UAM-Iztapalapa.

Por otra parte el hecho de haber encontrado varios taxa tanto de diatomeas, como de dinoflagelados, reconocidos como especies oceánicas, incluso algunas de las cuales han sido localizadas en zonas oceánicas muy profundas, es indicativo de la intrusión de agua oceánica a la región de estudio, posiblemente por su cercanía de la Trinchera Mesoamericana y por efecto del patrón de corrientes modelado para la bahía, el cual produce la entrada de una corriente subsuperficial por la porción occidental, que circula en su interior y sale por la parte oriental. Esta mezcla de masas de aguas costeras y oceánicas, contribuye a sumar elementos de comunidades de distinta estirpe, lo que conlleva a explicar la alta riqueza fitoplanctónica encontrada en su interior; pero además la intrusión de agua oceánica más oligotrófica, puede influir también en el estado trófico de la bahía, posibilitando la expresión de un mayor número de especies, en lugar de la dominancia permanente de solo algunas, como ocurre en cuerpos de agua eutróficos.

No obstante lo anterior, con base en la idea de Margalef (1978), acerca de que en el ambiente marino, las colonias o cenobio mucilaginosos se desarrollan primordialmente en condiciones de eutrofización, debido a que el mucílago pone resistencia a la difusión de los nutrientes desde el ambiente hasta las células. El hallazgo de los taxa Phaeocystis sp. y Planktoniella muriformis en la bahía, indican también que en ciertos momentos las concentraciones de nutrientes y materia orgánica en la región de estudio son altas (aunque esto no siempre se refleje en las concentraciones de nutrientes medidas en el agua).

La eutrofización de la bahía de Acapulco ocurre principalmente en la época de lluvias, debido a las descargas de arroyos temporales de aguas negras que bajan desde las montañas. Lo anterior es evidente al observarse que los niveles de fosfatos se elevan considerablemente justo en los meses de julio-agosto (época de lluvias), mientras que la salinidad disminuye, y no es sino hasta el inicio de las secas que se incrementan las formas nitrogenadas. 
Los procesos de eutrofización son importantes considerando los riesgos ambientales y a la salud provocados por los FAN, dado el elevado número de especies potencialmente nocivas registradas en la bahía de Acapulco (50 taxa), así como el registro de 23 taxa formadores de florecimientos entre los cuales hay especies consideradas como tóxicas o nocivas, tanto de dinoflagelados (Gymnodinium catenatum, Pyrodinium bahamense var. compressum, Akashiwo sanguinea, Cochloldinum polykrikoides y Karenia spp.) como de diatomeas (Pseudo-nitzschia spp.).

Asimismo el hallazgo de especies de Euglenophyta, como Euglena pascheri, indicadora de presencia de materia orgánica, asociada a la localidad "La Naval", en la cual el tiempo de resilencia del agua es mayor, es indicativo de la entrada de aguas negras a la bahía. Por el contrario, el hallazgo de relaciones simbióticas de cianofitas fijadoras de nitrógeno en o sobre dinoflagelados y diatomeas, así como del consorcio Leptocilindrus mediterraneus-Solenicola setigera, podrían por el contrario indicar limitaciones de compuestos nitrogenados, especialmente nitratos, en ciertos momentos.

Las anteriores condiciones contrastantes permiten suponer que los niveles de eutrofización en la bahía seguramente serian más elevados de no ser por un efecto combinado entre el "lavado" que juegan las corrientes marinas que entran y salen de la bahía, y el papel depurador que juega su comunidad fitoplanctónica. Ello nos lleva a plantear la hipótesis de que la bahía de Acapulco presenta una alta capacidad de resilencia, entendida como aquello que permite a un sistema, dentro de ciertos límites, regresar a un estado anterior después de sufrir una perturbación, es decir la capacidad que posee un ecosistema para absorber el estrés y poder seguir proporcionando beneficios. Lo que aquí resulta más interesante, es que se ha sugerido que la resilencia depende, al menos en parte, de la diversidad de los organismos y la heterogeneidad de sus funciones biológicas (Anónimo, 1996), por ello podemos inferir que la alta riqueza fitoplanctónica de Acapulco, no solo vista como el alto número de especies registradas, sino también como su complejidad en términos de la alta diversidad de funciones, considerando el predominio de dinoflagelados y la diversidad de tipos de nutrición y relaciones interespecíficas (autótrofos, mixótrofos, heterótrofos, simbiontes y parásitos), y de niveles de organización, debe estar relacionada con su alta capacidad de resilencia. Lo anterior marca una línea de investigación importante a seguir en este tenor, con la idea de que a través del estudios florísticos, en combinación con aspectos ecológicos de las comunidades y ambientales del sistema, dar elementos para conocer el estado de salud de la bahía en su justa dimensión y propiciar su conservación, disminuyendo la intensidad de los factores que causan su perturbación. Tales acciones requieren cierta urgencia, 
porque se ha señalado que la estabilidad en un sistema de transición, como lo es la bahía de Acapulco, se vuelve frágil por los acelerados cambios originados por el hombre y cuando dichas perturbaciones traspasan cierto umbral, provocando cambios cualitativos en las comunidades, los impactos se vuelven irreversibles (Anónimo, 1996).

\section{AGRADECIMIENTOS}

A la Comisión Nacional para el Conocimiento y Uso de la Biodiversidad por el apoyo financiero recibido para la realización de los proyectos de investigación: H176, S151, BA008, DJ022 y HJ014. Al Consejo Nacional de Ciencia y Tecnología por el apoyo financiero al proyecto de investigación G37560 y por la beca doctoral otorgada a la M. en C. M. E. Zamudio-Resendiz, ya que parte de los resultados del trabajo corresponden a su investigación doctoral en el Programa de Doctorado en Ciencias Biológicas y de la Salud de la Universidad Autónoma Metropolitana. A los integrantes del Laboratorio de Ecosistemas Costeros de la UAM-Iztapalapa, por la ayuda en la recolecta y evaluación de parámetros físico-químicos. Al M. en C. Gilberto Hernández Cárdenas por la figura del mapa de la bahía incluyendo la topografía de la región. Al Dr. Saúl López Silva, ex Director del Laboratorio Estatal de Salud Pública del Estado de Guerrero "Dr. Galo Soberón y Parra", por el apoyo logístico brindado para la realización de colectas en la Bahía de Acapulco. A los revisores anónimos por mejorar substancialmente el escrito.

\section{LITERATURA CITADA}

Achatz, J. G., M. Hooge, A. Wallberg, U. Jondelius \& S. Tyler. 2009. Systematic revision of acoels with $9+0$ sperm ultrastructure (Convolutida) and the influence of sexual conflict on morphology. J. Zool. Syst. Evol. Res. DOI: 10.1111/j.1439-0469.2009.00555.x

Aguilar-Camacho, J. M. 2010. Catálogo taxonómico y descripción de las esponjas de la bahía de Acapulco, Gro. (Pacífico Mexicano). Tesis de licenciatura. Universidad Autónoma de Guerrero, Unidad Académica de Ecología Marina. Acapulco, Guerrero, México. $115 \mathrm{pp}$.

Aké-Castillo, J. A. 1997. Sistemática y taxonomía de Chaetoceros Ehrenberg y Thalassiosira Cleve (Bacillariophyceae) del Golfo de Tehuantepec, México. Tesis de maestría. Instituto Politécnico Nacional, Centro de Investigación y de Estudios Avanzados, Unidad Mérida. Mérida, Yucatán, México. 84 pp. 
Aké-Castillo, J. A., D. U. Hernández-Becerril \& M. E. Meave-del Castillo. 1999. Species of the genus Thalassiosira (Bacillariophyta) from the Gulf of Tehuantepec, Mexico. Bot. Mar. 42: 487-503.

Alves-de-Souza, C., M. T. González \& J. L. Iriarte. 2008. Functional groups in marine phytoplankton assemblages dominated by diatoms in fjords of southern Chile. J. Plankton Res. 30(11): 1233-1243.

Anónimo. 1979. Estudio sobre la contaminación de agua en la Bahía de Acapulco y proximidades (Perfiles y distribución de temperatura, salinidad y oxígeno disuelto). Secretaría de Marina. México, D.F., México. 79 pp.

Anónimo. 1996. Características específicas de la diversidad biológica y sugerencias a las instituciones financieras sobre como lograr que sus actividades respalden más adecuadamente los objetivos del Convenio. Convenio Sobre la Diversidad Biológica. Programa de las Naciones Unidas para el Medio Ambiente. Tercera Reunión. Buenos Aires, Argentina. 9 pp. http://www.iisd.ca/biodiv/cop3/cop307s.pdf

Anónimo. 2005. Cuaderno estadístico municipal Acapulco de Juárez, Estado de Guerrero. Instituto Nacional de Estadística, Geografía e Informática. http://www.inegi.gob.mx/ est/contenidos/espanol/sistemas/cem05/estatal/gro/m001/index.htm

Anónimo. 2010a. Resultados de Saxitoxinas en Moluscos Bivalvos al 04 de Diciembre 2010. Laboratorio Estatal de Salud Pública, Guerrero, Subsecretaría de Regulación, Control y Fomento Sanitario. Acapulco, Guerrero, México. 3 pp. http://www. lesp-guerrero.gob.mx/wp-content/uploads/2010/07/Resultados-de-Saxitoxinas-al04DICIEMBRE10.pdf

Anónimo. 2010b. Clima en Acapulco. Est. Meteor. 768050 (Coordenadas: Latitud: $+16^{\circ} 83$ N, Longitud: $-099^{\circ} 93$ W). Alpred, S. L. Murcia, España. http://clima.tiempo.com/ clima-en-acapulco-768050-2010.

Balech, E. 1988. Los dinoflagelados del Atlántico Sudoccidental. Publ. Esp. Instit. Esp. Oceanogr. 1: 1-310.

Balech, E. 1995. The genus Alexandrium Halim (Dinoflagellata). Sherkin Island Marine Station. Co Cork, Ireland, UK. 151 pp.

Balleza-García, M. \& F. J. Néstor-Cervantes. 1992. Contribución al estudio de los quetognatos de la Bahía de Acapulco, Gro. Tesis de licenciatura. Universidad Autónoma de Guerrero, Escuela Superior de Ecología Marina. Acapulco, Guerrero, México. 104 pp.

Band-Schmidt, C. J., L. Morquecho, D. U. Hernández-Becerril, A. Reyes-Salinas \& E. Bravo-Sierra. 2004. Raphidophyceasn on the coast of Mexico. Hydrobiologia 515: 79-89.

Barba-Marino, F. 2009. Composición y zonificación de la comunidad de moluscos de sustrato rocoso en dos sitios con distinta acción de oleaje, en la isla "La Roqueta", Acapulco, Guerrero, México. Tesis de licenciatura. Universidad Autónoma de Guerrero, Unidad Académica de Ecología Marina. Acapulco, Guerrero, México. 179 pp.

Bérard-Therriault, L., M. Poulin \& L. Bossé. 1999. Guide d'ídentifcation du phytoplankton marin de l'estuaire et du golfe du Saint-Laurent. Conseil National de Recherches du Canada. Ottawa, Canadá. 387 pp. 
Blanchot, J. \& M. Rodier. 1996. Picoplankton abundances in the western tropical Pacific Ocean during the 1992 El Niño year: results from flow cytometry. Deep Sea Res. 43(6): 877-895.

Bourrelly, P. 1972. Les algues d'eau douce, initiation a la systématique. Tome I: Les Algues Vertes. Editions N. Boubée Cie. París, Francia. 572 pp.

Bravo-Sierra, E. 1999. Composición del fitoplancton de red en Bahía Banderas, México, 1990-1991. Tesis de maestría. Facultad de Ciencias, Universidad Nacional Autónoma de México. México, D.F., México. 104 pp.

Buck, K. R. \& W. N. Bentham. 1998. A novel symbiosis between a cyanobacterium, Synechococcus sp., an aplastidic protist, Solenicola setigera, and a diatom, Leptocylindrus mediterraneus, in the open ocean. Mar. Biol. 132: 349-355.

Cárdenas-Santiago, M. H \& B. Jiménez-Escobar. 2001. Diversidad de aves marinas en la Bahía de Acapulco, Gro. México. Tesis de licenciatura. Universidad Autónoma de Guerrero, Escuela Superior de Ecología Marina. Acapulco, Guerrero, México. 55 pp.

Clarke, K. R. \& R. N. Gorley. 2006. Multivariate statics for ecologists PRIMER v6. Plymouth, UK. 192 pp.

Cortés-Altamirano, R. \& N. Pasten-Miranda. 1982a. Composición, abundancia y distribución del fitoplancton del Estero de Urías, Sin., México. I. Período primaveral 1980. Rev. Lat. Microbiol. 24: 103-114.

Cortés-Altamirano, R. \& N. Pasten-Miranda. 1982b. Composición, abundancia y distribución del fitoplancton del estero Urías, Sin., México. II. Período Verano 1980. Rev. Lat. Microbiol. 24: 297-308.

Cortés-Altamirano, R. \& N. Pasten-Miranda. 1985. Composición, abundancia y distribución del fitoplancton del Estero de Urías, Sin. México. IV. Período de Invierno. Rev. Lat. Microbiol. 27: 123-133.

Cronberg, G. E, J. Carpenter \& W. W. Carmichael. 2004. Taxonomy of harmful cyanobacteria. In: Hallegraeff, G. M., D. M. Anderson y A. D. Cembella (eds.). Manual on Harmful Marine Microalgae. 2a. ed. United Nations Educational Scientific and Cultural Organization Publishing. París, Francia. pp. 523-562.

Dodson, A. N. \& W. H. Thomas. 1978. Reverse filtration. In: Sournia, A. (ed.). Phytoplankton Manual. United Nations Educational Scientific and Cultural Organization. París, Francia. pp. 104-107.

Domínguez-Parra, S. 1979. Estudio de la calidad reglamentaria y ecológica de las aguas costeras en la Bahía de Acapulco, Gro. y proximidades realizado de noviembre de 1978 a marzo de 1979. Tesis de maestría en ciencias. Facultad de Ciencias, Universidad Nacional Autónoma de México. México, D.F., México. 153 pp.

Edler, L. \& M. Elbrächter. 2010. 2 The Utermöhl method for quantitative phytoplankton analysis. In: Karlson, B., A. Cusack \& E. Bresnan (eds.). Microscopic and molecular methods for quantitative phytoplankton analyses. United Nations Educational Scientific and Cultural Organization. París, Francia. pp. 13-20.

Elbrächter, M. 1979. On the taxonomy of unarmored dinophytes (Dinophyta) from the Northwest African upwelling region. Meteor Forschungs Reihe 30: 1-22.

Emery, K. O. 1967. Estuaries and Lagoons in relation to continental shelves. Biol. Bull. 1493: 9-11. 
Estradas-Romero, A. R., M. Prol-Ledesma \& M. E. Zamudio-Reséndiz. 2009. Relación de las características geoquímicas de los fluidos hidrotermales con la abundancia y riqueza de especies del fitoplancton de Bahía Concepción, Baja California Sur, México. Bol. Soc. Geol. Mex. 61(1): 87-96.

Fensome, R. A., F. J. R. Taylor, G. Norris, W. A. S. Sarjeant, D. I. Wharton \& G. L. Williams. 1993. A classification of living and fossil Dinoflagellates. Micropaleontology 7: 1-351.

Flamand-Swaner, C. L. 1991. Pacífico tropical mexicano, Cabo Corrientes a la frontera con Guatemala. Oceanografía Geológica. In: De la Lanza Espino, G. (comp.). Oceanografía de mares mexicanos. AGT Editor. México, D.F., México. pp. 117-148.

Fritz, L. \& R. E. Triemer. 1985. A rapid simple technique utilizing Calcofluor white M2R for the visualization of Dinoflagellate thecal plates. J. Phycol. 21: 662-664.

Fryxell, G. A. \& G. R. Hasle. 2004. Taxonomy of harmful diatoms. In: Hallegraeff, G. M., D. M. Anderson, A. D. Cembella (eds.). Manual on Harmful Marine Microalgae. 2a. ed. United Nations Educational Scientific and Cultural Organization Publishing. París, Francia. pp. 465-510.

Gaines, G. \& M. Elbrächter. 1987. Heterotrophic nutrition. In: Taylor, F. J. R. (ed.). The Biology of Dinoflagellates. Bot. Monogr. 21: 224-268.

Gárate-Lizárraga, I. 1989. Nuevos registros de especies del género Rhizosolenia en la región central del golfo de California y Bahía Magdalena, B.C.S. CICIMAR 4(2): 291-296.

Gárate-Lizárraga, I. 1991. Análisis de una marea roja causada por Noctiluca scintillans (Macartney) Ehr. en Bahía Concepción Baja California Sur en febrero de 1989. Rev. Invest. Cien. 2(1): 35-43.

Gárate-Lizárraga, I. 1992. Variación espacio temporal de la abundancia fitoplanctónica y de la estructura de las asociaciones microfitoplanctónicas en el Sistema Lagunar Magdalena-Almejas durante 1984-1986. Tesis de maestría. Centro Interdisciplinario de Ciencias del Mar, Instituto Politécnico Nacional. La Paz, Baja California Sur, México. 84 pp.

Gárate-Lizárraga, I. 2008. Nuevos registros de dinoflagelados planctónicos para el Golfo de Tehuantepec, México. Oceánides 23 (1,2): 55-65.

Gárate-Lizárraga, I. 2009. First record of Ceratium dens (Dinophycae) in the Gulf of California. Oceánides 24(2): 167-173.

Gárate-Lizárraga, I. \& R. González-Armas. 2011. Occurrence of Pyrodinium bahamense var. compressum along the southern coast of Baja California Peninsula. Mar. Pollut. Bull. 62: 626-630.

Gárate-Lizárraga, I. \& S. Muñetón-Gómez. 2009. Primer registro de la diatomea epibionte Pseudohimantidium pacificum y de otras asociaciones simbióticas en el Golfo de California. Acta Bot. Mex. 88: 31-45.

Gárate-Lizárraga, I. \& G. Verdugo-Díaz. 2007. Nuevos registros de dinoflagelados desnudos para el Golfo de California, México. Oceánides 22(1-2): 37-43.

Gárate-Lizárraga, I., C. J. Band-Schmidt, G. Verdugo-Díaz, M. S. Muñetón-Gómez \& E. F. Félix-Pico. 2007. Dinoflagelados (Dinophyceae) del sistema lagunar MagdalenaAlmejas. In: Funes-Rodríguez, R., J. Gómez-Gutiérrez, R. Palomares-García (eds.). Estudios ecológicos en Bahía Magdalena. Centro Interdisciplinario de Ciencias del Mar, Instituto Politécnico Nacional. La Paz, Baja California Sur, México. pp. 145-174. 
Gárate-Lizárraga, I., C. J. Band-Schmidt, F. Aguirre-Baena \& T. Grayev del Álamo. 2009. A multi species microalgae Bloom in Bahía de La Paz, Gulf of California, Mexico (June 2008). Oceánides 24(1): 1-15.

Gárate-Lizárraga, I., R. E. Muciño-Márquez \& G. Verdugo-Díaz. 2010. First record of Erythropsidinium agile (Gymnodiniales: Warnowiaceae) in the Mexican Pacific. Oceánides 25(2): 137-142.

Gárate-Lizárraga, I., A. Díaz-Ortíz, B. Pérez-Cruz, M. A. Alarcón-Romero, L. A. ChávezAlmazán, J. L. García-Barbosa \& S. López-Silva. 2011. A multi-species dinoflagellate Bloom and shellfish toxicity in Costa Grande, Guerrero, Mexico (December, 2010). Oceánides 26(1): 61-71.

Gárate-Lizárraga, I., B. Pérez-Cruz, J. A. Díaz-Ortíz, M. Alarcón-Tacuba, L. A. ChávezAlmazán, M. A. Alarcón-Romero, S. López-Silva, J. J. Bustillos-Guzmán \& S. Licea-Durán. 2012. Toxicity and paralytic toxin profile in Pyrodinium bahamense var. compressum and violet oyster in Bahía de Acapulco. Harmful Algae News 45: 2-3.

García-López, J. A. 1994. Fauna malacológica de acompañamiento del caracol Purpura pansa Gould 1853, en la zona mesolitoral de la Isla la Roqueta, Acapulco, Gro., México. Tesis de licenciatura. Universidad Autónoma de Guerrero, Escuela Superior de Ecología Marina. Acapulco, Guerrero, México. 97 pp.

Gómez, F. 2007. The consortium of the Protozoan Solenicola setigera and the diatom Leptocylindrus mediterraneus in the Pacific Ocean. Acta Protozool. 46: 15-24.

Gómez, F. 2008. Erythropsidinium (Gymnodiniales, Dinophyceae) in the Pacific Ocean, a unique dinoflagellate with an ocelloid and a piston. Eur. J. Protistol. 44: 291-298.

Gómez, F. 2010. Neoceratium gen. nov., a new genus for all marine species currently assigned to Ceratium. Protist 161(35-54): 35-54.

Hallegraeff, G. M. 2004. Harmful algal blooms: a global overview. In: Hallegraeff, G. M., D. M. Anderson, A. D. Cembella (eds.). Manual on Harmful Marine Microalgae. 2a. ed. United Nations Educational Scientific and Cultural Organization Publishing. París, Francia. pp. 25-50.

Hallegraeff, G. M. \& Y. Hara. 2004. Taxonomy of harmful marine raphidophytes. In: Hallegraeff, G. M., D. M. Anderson, A. D. Cembella (eds.). Manual on Harmful Marine Microalgae. 2a. ed. United Nations Educational Scientific and Cultural Organization Publishing. París, Francia. pp. 511-522.

Hansen, P. J. 1991. Quantitative importance and trophic role of heterotrophic dinoflagellate in a coastal pelagic web. Mar. Ecol. Prog. Ser. 73: 253-261.

Hasle, G. R. 1975. Some living marine species of the diatom family Rhizosoleniaceae. Beih. Nova Hedwigia 53: 99-140.

Hasle, G. R. 1978. Some specific preparations. Diatoms. In: Sournia, A. (ed.). Phytoplankton Manual. United Nations Educational Scientific and Cultural Organization. Paris, France. pp. 137-142.

Hasle, G. R. \& E. E. Syvertsen. 1997. Marine Diatoms. In: Tomas, C. R. (ed.). Identifying marine phytoplankton. Acad. Press Inc. California, USA. pp. 5-385.

Heimdal, B. R. 1997. Modern coccolithophorids. In: Tomas, C. R. (ed.). Identifying Marine Phytoplankton. Acad. Press. Inc. California, USA. pp. 731-847. 
Hendricks, E. M. 1993. Crustáceos Decápodos del Pacífico Mexicano. In: Salazar Vallejo, S. I. \& N. E. González (eds.). Biodiversidad marina y costera de México. Comisión nacional para el Conocimiento y Uso de la Biodiversidad-Centro de Investigaciones de Quintana Roo. Chetumal, México. pp. 272-318.

Hernández-Becerril, D. U. 2003. La diversidad del fitoplancton marino de México. Un acercamiento actual. In: Barreiro-Güemes, M. T., M. E. Meave del Castillo, M. R. Signoret-Poillon \& M. G. Figueroa-Torres (eds.). Planctología Mexicana. Sociedad Mexicana de Planctología, A.C. México, D.F., México. pp. 1-17.

Hernández-Becerril, D. U. \& E. Bravo-Sierra. 2004a. Observation on rare planktonic dinoflagellate, Dinofurcula cf. ultima (Dinophyceae), from the Mexican Pacific. Phycol. 43(4): 341-345.

Hernández-Becerril, D. U. \& E. Bravo-Sierra. 2004b. New records of planktonic Dinoflagellates (Dinophyceae) from the Mexican Pacific Ocean. Bot. Mar. 47: 417423.

Hernández-Becerril, D. U. \& E. M. Diaz-Almeyda. 2006. The Nitzschia bicapitata group, new records of the genus Nitzschia, and further studies on species of Pseudonitzschia (Bacillariophyta) from Mexican Pacific coasts. Nova Hedwigia Beih. 130: 293-306.

Hernández-Becerril, D. U., M. E. Meave del C. y C. Flores Granados. 2003. Dinoflagelados del Orden Dinophysiales en las Costas Mexicanas. In: Barreiro, M. T., M. E. Meave del Castillo, G. Figueroa-Torres y M. Signoret (eds.). Planctología Mexicana. Sociedad Mexicana de Planctología, A.C. México, D.F., México. pp. 19-42.

Hernández-Becerril, D. U., J. G. A. Ceballos-Corona, K. Esqueda-Lara, M. A. TovarSalazar \& D. León-Álvarez. 2008. Marine Planktonic dinoflagellates of the order Dinophysiales (Dinophyta) from coasts of the tropical Mexican Pacific, including two new species of the genus Amphisolenia. J. Mar. Biol. Assoc. 88(1): 1-15.

Hernández-Becerril, D. U., P. Herrera-Hernández, A. Pérez-Mendoza \& J. G. A. Ceballos-Corona. 2010. Marine planktonic Diatoms of the order Rhizosoleniales (Bacillariophyta) from the tropical Mexican Pacific. Vie Milieu 60(2): 95-107.

Hoek, C. van den; D. G. Mann \& H. M. Jahns. 1995. Algae. An introduction to phycology. Cambridge University Press. Cambridge, USA. 623 pp.

Huber-Pestalozzi, G. 1955. Das Phytoplankton des Sübwasser: Systematik und Biologie. Tomo 4. Euglenophycean. E. Schweizerbart'sche Verlagbuchhandiung. Stüttgart, Alemania. 606 pp., 114 láms.

Itakura, S. \& M. Yamaguchi. 2001. Germination characteristics of natural occurring cysts of Alexandrium tamarense (Dinophyceae) in Hiroshima Bay, Inland Sea Japan. Phycologia 40(3): 263-267.

Johnson, M. D. \& D. K. Stoecker. 2005. Role of feeding in growth and photophysiology of Myrionecta rubra. Aquat. Microb. Ecol. 39: 303-312.

Kofoid, C. A. \& O. Swezy, 1921.The free-living unarmored Dinoflagellata. Memories of University of California. Vol. 5. University of California Press. Berkeley, USA. 562 pp.

Komárek, J. \& K. Anagnostidis. 2005. Cyanoprokaryota 2. Teil/ 2nd Part: Oscillatoriales. In: Büdel, B., L. Krienitz, G. Gärtner \& M. Schagerl (eds.). Süsswasserflora von Mitteleuropa 19/2. Elsevier/Spektrum. Heidelberg, Alemania. 759 pp. 
Krayesky, D. M., E. Meave del C., E. Zamudio, J. N. Norris \& S. Fredericq. 2009. Diatoms (Bacillariophyta) of the Gulf of Mexico. In: Felder, D. L. \& D. K. Camp (eds.). Gulf of Mexico origin, waters, and biota. Vol.1. Biodiversity. Texas A \& M University Press. College Station, Texas, USA. pp. 155-186.

Kulm, L. D., C. Roush, C. Harlett, H. Heudeck, H. Chambers \& J. Runge. 1975. Oregon Continental Shelf Sedimentation Interrelationships of facies distribution and sedimentary processes. J. Geol. 83(2): 145-175.

Lansberg, J. H. \& K. A. Steidinger. 1998. A historical review of Gymnodinium breve red tides implicated in mass mortalities of the manatee (Trichechus manatus latirostris) in Florida, USA. In: Reguera, B., J. Blanco, M. L. Fernández \& T. Wyatt (eds.). Harmful algae. Comisión Oceanográfica Intergubernamental, United Nations Educational, Scientific and Cultural Organization. París, Francia. pp. 97-100.

Lee, R. E. 1999. Phycology. Cambridge University Press. Cambridge, USA. 614 pp.

Licea, S., J. L. Moreno-Ruiz, H. Santoyo-Reyes \& G. Figueroa. 1995. Dinoflageladas del Golfo de California. Universidad Autónoma de Baja California Sur, Secretaria de Educación Pública, Fondo para Modernización de la Educación Superior, Programa Interdisciplinario e Interinstitucional Mar de Cortés. México, D.F., México. 165 pp.

Ligeza, S. \& E. Wilk-Wozniak. 2011. The occurrence of a Euglena pascheri and Lepocinclis ovum bloom in an oxbow lake in southern Poland under extreme environmental conditions. Ecol. Indic. 11: 925-929.

Lluch-Cota, S. E. 2000. Coastal upwelling in the Eastern Gulf of California. Oceanologica Acta 23(6): 731-740.

Lluch-Cota, S. E., S. Álvarez-Borrego, E. M. Santamaría del Ángel, F. E. Müller-Karger \& S. Hernández-Vázquez. 1997. The Gulf of Tehuantepec and adjacent areas: spatial and temporal variation of satellite-derived photosynthetic pigments. Ciencias Marinas 23(3): 329-340.

Lucero-Blanco, A. \& G. A. Herrera-Hernández. 2007. Distribución espacio temporal de dos especies de erizos de mar: Echinometra vanbrunti y Diadema mexicanum, en la zona infralitoral de la isla La Roqueta, Acapulco, Gro., México. Tesis de licenciatura. Universidad Autónoma de Guerrero, Unidad Académica de Ecología Marina. Acapulco, Guerrero, México. 47 pp.

Magurran, A. E. 2004. Measuring biological diversity. Blackwell Publishing. Oxford, UK. $256 \mathrm{pp}$.

Margalef, R. 1978. Life-forms of phytoplankton as survival alternatives in an unstable environment. Oceanológica Acta 1(4): 493-509.

Martínez Orozco, S. Y. 2004. Estudio de la productividad de la población de los copépodos Pelágicos de la Bahía de Acapulco Guerrero, México. Tesis de licenciatura. Universidad Autónoma de Guerrero, Unidad Académica de Ecología Marina. Acapulco, Guerrero, México. 39 pp.

Mayo-Vera, A. B. 2004. Estudio Ambiental de la bahía de Acapulco, Guerrero. Tesis de licenciatura (Ingeniero Geólogo).Universidad Nacional Autónoma de México, Facultad de Ingeniería. México, D.F., México. 76 pp.

Meave-del Castillo, M. E. 1999. Diatomeas planctónicas del Pacífico Mexicano. Informe final Sistema Nacional de Información sobre Biodiversidad de México-Comisión 
Nacional para el Conocimiento y Uso de la Biodiversidad proyecto Proyecto H176. México, D.F., México. 487 pp. http://www.conabio.gob.mx/institucion/proyectos/ resultados/InfH176\%20primera\%20parte.pdf

Meave-del Castillo, M. E. 2002. Diatomeas marinas planctónicas de la zona costera del Pacífico tropical mexicano. Informe final Sistema Nacional de Información sobre Biodiversidad de México-Comisión Nacional para el Conocimiento y Uso de la Biodiversidad proyecto Proyecto S151. México, D.F., México. 37 pp. http://www. conabio.gob.mx/institucion/proyectos/resultados/InfS151.pdf

Meave-del Castillo, M. E. 2006. Diatomeas (Bacillariophyta), Dinoflagelados (Dinophyta) y Silicoflagelados (Dictyochophyceae) Marinos del Pacífico Mexicano, con énfasis en la porción tropical. Informe final Sistema Nacional de Información sobre Biodiversidad de México-Comisión Nacional para el Conocimiento y Uso de la Biodiversidad proyecto Proyecto BA008. México, D.F., México. 80 pp. http://www.conabio.gob.mx/ institucion/proyectos/resultados/InfBA008\%20parte\%201.pdf

Meave-del Castillo, M. E. 2009. Dinoflagelados y diatomeas del Pacífico tropical mexicano. Informe final Sistema Nacional de Información sobre Biodiversidad de MéxicoComisión Nacional para el Conocimiento y Uso de la Biodiversidad proyecto Proyecto DJ022. México, D.F., México. http://www.conabio.gob.mx/institucion/ proyectos/resultados/InfDJ022.pdf

Meave-del Castillo, M. E. \& D. U. Hernández-Becerril. 1998. 6 Fitoplancton. El Golfo de Tehuantepec: el ecosistema y sus recursos. In: Tapia García, M. Universidad Autónoma Metropolitana - Iztapalapa. México, D.F., México. pp. 59-74.

Meave-del Castillo, M. E., C. A. Aké, M. E. Zamudio y M. S. Guerra. 2001. Registro de Diatomeas Planctónicas de las Costas del Estado de Nayarit, México (julio, 1999). Scientiae Naturae 3(2): 5-23.

Meave-del Castillo, M. E., M. E. Zamudio-Resendiz, J. A. Aké Castillo, S. Guerra-Martínez y I. F. Barbosa Ledesma. 2003a. Biodiversidad de Diatomeas (Bacillariophyta) en la Columna de Agua del Pacífico Mexicano. In: Barreiro, T., M. E. Meave del Castillo, G. Figueroa-Torres y M. Signoret (eds.). Planctología Mexicana. Sociedad Mexicana de Planctología, A.C. México, D.F., México. pp. 43-84.

Meave-del Castillo, M. E., M. E. Zamudio-Resendiz, Y. B. Okolodkov \& I. H. Salgado Ugarte. 2003b. Ceratium balechii sp. nov. (Dinophyceae: Gonyaulacales) from the Mexican Pacific. Hidrobiológica 13(1):75-91.

Meave-del Castillo, M. E., M. E. Zamudio-Resendiz \& L. F. Fernández. 2008. Fryxelliella sepulvedana sp. nov. (Triceratiaceae: Bacillariophyta) a rare species from the Mexican Pacific coasts. Iheringia, Ser. Bot. 63(1): 177-185.

Mendes-Lopes, R. \& M. Silveira. 1994. Symbiosis between a pelagic flatworm and a dinoflagellate from a tropical area: structural observations. Hydrobiologia 287: 277-284.

Moestrup, O. \& H. Thompsen. 2004. Taxonomy of toxic Haptophytes (Primmnesiophytes). In: Hallegraeff, G. M., D. M. Anderson, A. D. Cembella (eds.). Manual on Harmful Marine Microalgae. 2a. ed. United Nations Educational Scientific and Cultural Organization Publishing. París, Francia. pp. 433-464.

Moreno, J. L., S. Licea y H. Santoyo. 1996. Diatomeas del Golfo de California. Universidad Autónoma de Baja California Sur. Secretaria de Educación Pública, Fondo 
para Modernización de la Educación Superior, Programa Interdisciplinario e Interinstitucional Mar de Cortés. México, D.F., México. 273 pp.

Moreno-Gutiérrez, S. P. 2008. Estudio morfológico y taxonómico de diatomeas planctónicas del género Pseudonitzschia Peragallo (Bacillariophyceae) en el Golfo de Tehuantepec, México. Tesis de licenciatura. Facultad de Estudios SuperioresZaragoza, Universidad Nacional Autónoma de México. México, D.F., México. 61 pp.

Morquecho, L. \& C. H. Lechuga-Devéze. 2004. Seasonal occurrence of planktonic dinoflagellates and cyst production in relationship to enviromental variables in subtropical Bahía Concepción, Gulf of California. Bot. Mar. 47: 313-322.

Murphy, J. \& P. Riley. 1962. A modified single solution method for the determination of phosphate in natural waters. Anal. Chem. Acta 27: 31-36.

Nava-Cuevas, L. E. 2008. Análisis comparativo de doce años (1995-2006) de las actividades de protección y conservación de la tortuga marina, especie Golfina (Lepidochelys olivacea), en los campamentos tortugueros de Santa Cruz de Mitla, municipio de Coyuca de Benítez y playa Boca Chica, municipio de Tecpan de Galeana, estado de Guerrero, México. Tesis de licenciatura. Universidad Autónoma de Guerrero, Unidad Académica de Ecología Marina. Acapulco, Guerrero, México. 87 pp.

Okolodkov, Y. B. \& I. Gárate-Lizárraga. 2006. An annotated checklist of Dinoflagellates (Dinophyceae) from the Mexican Pacific. Acta Bot. Mex. 74: 1-154.

Orellana, E., E. Martínez, L. Muñoz, P. López, E. Cabrera \& C. Ramírez. 1998. Toxicity associated with blooms of Pyrodinium bahamense var. compressum in southwestern Mexico. In: Reguera, B., J. Blanco, M. Fernández \& T. Wyatt (eds.). Harmful algae. Harmful algae. Comisión Oceanográfica Intergubernamental, United Nations Educational, Scientific and Cultural Organization. París, Francia. pp. 60.

Osorio-Tafall, B. F. 1942. Notas sobre algunos dinoflagelados planctónicos marinos de México, con descripción de nuevas especies. An. Esc. Nac. Cienc. Biol. 2: 435-450.

Pacheco-Sandoval, P. 1991. Pacífico tropical mexicano, Cabo corrientes a la frontera con Guatemala. Oceanografía Física. In: de la Lanza Espino, G. (Comp.). Oceanografía de mares mexicanos. AGT Editor. México, D.F., México. pp. 151-175.

Palmer, M. W. 1990. The estimation of species richness by extrapolation. Ecology 71(3): 1195-1198.

Parsons, T., C. Maita \& C. Lally. 1984. A manual of Chemical and Biological Methods of Seawater Analysis. Pargamon Press. Oxford, UK. 173 pp.

Porter, S. D. 2008. Algal Attributes: an autecological classification of algal taxa collected by the National Water-Quality Assessment Program. U.S. Geological Survey Data Series 329. Virginia, USA. http://pubs.usgs.gov/ds/ds329

Prézelin, B. 1987. Photosynthetic physiology of dinoflagellates. In: Taylor, F. J. R. (ed.). The biology of Dinoflagellates. Blackwell Scientific Publications. Oxford, UK. pp. 174223.

Priego-Martínez, B. C. 1985. Contribución al conocimiento de la composición fitoplanctónica del Estero de Urías y la Bahía de Mazatlán, Sinaloa. Tesis de licenciatura. Facultad de Ciencias Naturales y Agropecuarias, Universidad Autónoma de Guadalajara. Guadalajara, Jalisco, México. 85 pp. 
Quijano-Scheggia, S., A. Olivos-Ortiz, J. H. Gaviño-Rodríguez, F. Castro-Ochoa, M. Rivera-Vilarelle, M. Galicia-Pérez \& M. Patiño-Barragán. 2011. First report of Pseudo-nitzschia brasiliana and P. micropora (Bacillariophyceae) found in Coyutlan lagoon, Mexico. Rev. Biol. Mar. Ocenogr. 46(2): 189-197.

Ramírez, S. J. M. 1987. Turismo y medio ambiente: El caso de Acapulco. Estudios Demográficos y Urbanos 2: 479-512.

Ramírez-Camarena, C., A. Martínez-García, N. Juárez-Ruiz, R. Rojas-Crisóstomo y H. Ramírez-García. 2004. Impactos de Pyrodinium bahamense var. compressum durante el florecimiento algal nocivo 2001-2002, en la costa suroeste de México. Abstracts, XIII Reunión Nacional de la Sociedad Mexicana de Planctonología, A.C. VI International Meeting of Planktonology, abril 25-28. Nuevo Vallarta, Nayarit, México. p. 62.

Ramírez-Rosas, S. 2004. Evaluación de la diversidad y abundancia de los copépodos pelágicos en la Bahía de Acapulco Gro, México. Tesis de licenciatura. Universidad Autónoma de Guerrero, Unidad Académica de Ecología Marina. Acapulco, Guerrero, México. 43 pp.

Reguera, B. 2002. Establecimiento de un programa de seguimiento de microalgas toxicas. In: Sar, E. A., M. E. Ferrario \& B. Reguera (eds.). Floraciones algales nocivas en el cono Sur Americano. Instituto Español de Oceanografía. Vigo, España. pp. 21-54.

Rendón-Gómez, A. 1995. Contribución al conocimiento de la Biología Pesquera poblacional de Huachinango (Lutjanus peru), en la Bahía de Acapulco, Gro., México. Tesis de licenciatura. Universidad Autónoma de Guerrero, Escuela Superior de Ecología Marina. Acapulco, Guerrero, México. 47 pp.

Reynolds, C. S. 1997. Vegetation Processes in the Pelagic: A Model for Ecosystem Theory. Ecology Institute. Oldendorf/Luhe, Alemania. $371 \mathrm{pp}$.

Rojas-Herrera, A. A., J. Violante-González, S. García-Ibáñez, V. M. G. Sevilla-torres, J. S. Gil-Guerrero \& P. Flores-Rodríguez. 2012. Temporal variation in the phytoplankton community of Acapulco Bay, Mexico. Microbiol. Res. 3(e4): 13-19.

Rojas-Trejo, S. 1984. Ciclo anual del fitoplancton de la Bahía de Mazatlán, Sinaloa, México (1980). 1-45. Tesis de licenciatura. Escuela de Biología, Universidad Autónoma del Estado de Morelos. Morelos, México. 20 pp.

Round, F. E., R. M. Crawford \& D. G. Mann. 1990. The Diatoms. Biology and morphology of the genera. Cambridge University Press. Cambridge, EUA. 747 pp.

Salcedo-Oropeza, D. L. 2011. Análisis taxonómico y aspectos ecológicos de los Silidos (Annelida, Polychaeta, Syllidae) asociados a sustratos blandos del oeste de la Bahía de Acapulco, Guerrero. Tesis de licenciatura. Facultad de Ciencias, Universidad Nacional Autónoma de México. México, D.F., México. 108 pp.

Schwartz, M. C. 1942. Photometric determination of silica in the presence of phosphates. Ind. Eng. Chem. An. Ed. 14: 893-895.

Simonsen, R. 1974. The diatom plankton of the Indian Ocean Expedition of RV "Meteor" 1964-1965. Meteor Forschungsergebnisse Reihe D, 19: 14, pls. 10-11.

Solórzano, L. 1969. Determination of ammonia in natural water by phenol-hypochlorite method. Limnol. Oceanog. 14: 799-801.

Steidinger, K. \& K. Tangen. 1997. Dinoflagellates. In: Tomas, C. R. (ed.). Identifying marine phytoplankton. Acad. Press, Inc. San Diego, USA. pp. 387-584. 
Sterrenburg, F. A. S., M. E. Meave-del Castillo \& M. A. Tiffany. 2003. Studies on the Genera Gyrosigma and Pleurosigma (Bacillariophyceae): Pleurosigma species in the plankton from the Pacific coast of México, with the description of $P$. gracilitatis sp. nov. Crypto. Algol. 24(4): 291-306.

Strickland, J. \& T. Parsons, 1972. A practical handbook of seawater analysis. Fisheries Research Board of Canada. Ottawa, Canadá. 310 pp.

Tamayo, J. L. 1970. Geografia Moderna de México. Ed. Trillas. México, D.F., México. 390 $\mathrm{pp}$.

Taylor, D. L. 1971. On the symbiosis between Amphidinium klebseii (Dinophyceae) and Amphiscolops langerhansi (Tubellaria:Acoela). J. Mar. Biol. Ass. U.K. 51: 301-313.

Taylor, F. J. R. 1976. Dinoflagellates from the International Indian Ocean Expedition. A report on material collected by the R. V. "Anton Bruun" 1963-1964. Biblioth. Bot. 132: 1-234.

Taylor, F. J. R. 1982. Symbioses in marine microplankton. Ann. Inst. Océanogr., Paris 8: 61-90.

Taylor, F. J. R., Y. Fukuyo, J. Larsen \& G. M. Hallegraeff. 2004. Taxonomy of Harmful Dinoflagellates. In: Hallegraeff, G. M., D. M. Anderson, A. D. Cembella (eds.). Manual on Harmful Marine Microalgae. 2a. ed. United Nations Educational Scientific and Cultural Organization Publishing. París, Francia. pp. 389-432.

Tett, P. \& E. D. Barton. 1995. Why are there about 5000 species of phytoplankyton in the sea?. J. Plank. Res. 17: 1693-1704.

Throndsen, J. 1978. Preservation and storage. In: Sournia, A. (ed.). Phytoplankton Manual United Nations Educational Scientific and Cultural Organization. París, Francia. pp. 69-74.

Throndsen, J. 1997. The planktonic Marine Flagellates. In: Tomas, C. R. (ed.). Identifying marine phytoplankton. Acad. Press, Inc. California, USA. pp. 591-729.

Transviña, C. A. 2002. La circulación costera del Pacífico tropical oriental, con énfasis en la Alberca Cálida mexicana. Colección Dialogo entre pares, Consejo Nacional de Acreditación. Bogotá, Colombia. 20 pp. bcs.cicese.mx/trasvi/ Biblio/12COLOMBIA2002publicado.pdf

Wolowski, K. \& F. Hindák. 2005. Atlas of Euglenophyta. VEDA Publishing House of the Slovak Academy of Science. Batislava, Slovakia. 136 pp.

Whittaker, R. H. 1959. On the Broad Classification of Organisms. Q. Rev. Biol. 34(3): 210226.

Zingone, A., M. J. Chrétiennot-Dinet, M. Lange \& L. Medlin. 1999. Morphological and genetic characterization of Pheocystis cordata and P. jahnii (Prymnesiophyceae), two new species from the Mediterranean Sea. J. Phycol. 35: 1322-1337. 


\section{APÉNDICE 1}

Relación de los 641 taxa de microalgas registradas en la columna de agua de Bahía de Acapulco y zona costera aledaña (por división y dentro de cada una de ellas en orden alfabético). Sobre el nombre con un $(*)$ se indica la potencialidad de nocividad y con (**) la potencialidad de toxicidad. Se presenta: Tipo de muestreo (M): $\mathrm{R}=$ Red, $\mathrm{B}=$ Botella, $\mathrm{V}=$ Viva. Frecuencia de aparición (Frec.). Tipo de nutrición $(\mathbf{N}): \mathrm{F}=$ Fotosintética, $\mathrm{H}=$ Heterótrofa, $\mathrm{M}=$ Mixótrofa. Forma de vida $(\mathbf{H})$ : T $=$ Ticoplanctónico, $\mathrm{P}=$ Planctónico. Nivel de organización (NO): 1 = Unicelular móvil o flagelada, 2 = Unicelular no flagelada, 3 = Colonia-cenobio, $4=$ Filamento-cadena. Distribución global: $\mathrm{Tr}=$ tropical, $\mathrm{Sbt}=$ subtropical, $\mathrm{Tm}=$ en aguas templadas, $\mathrm{Fr}=$ en aguas frías. Afinidad: $\mathrm{Slb}=$ salobre, $\mathrm{Ner}=$ Nerítico, $\mathrm{Oc}=$ Oceánico), y valores máximos y mínimos de abundancia (céls. L-1) para cada taxon. Los taxa infraespecíficos del género Ceratium no han sido transferidos formalmente al género Neoceratium, por esa razón en el listado para esos taxa el género se nombra de la siguiente manera: Neoceratium (Ceratium).

\begin{tabular}{|c|c|c|c|c|c|c|c|c|}
\hline \multirow[b]{2}{*}{ Taxa } & \multirow[b]{2}{*}{$\mathrm{M}$} & \multirow[b]{2}{*}{ Frec. } & \multirow[b]{2}{*}{$\mathrm{N}$} & \multirow[b]{2}{*}{$\mathrm{H}$} & \multirow[b]{2}{*}{ NO } & \multirow{2}{*}{$\begin{array}{l}\text { Distribución } \\
\text { Global }\end{array}$} & \multicolumn{2}{|c|}{$\begin{array}{l}\text { Abundancia } \\
\text { céls. } L^{-1}\end{array}$} \\
\hline & & & & & & & Mín. & Máx. \\
\hline \multicolumn{9}{|l|}{ División Bacillariophyta } \\
\hline Achnanthes brevipes Agardh & $\mathrm{R}, \mathrm{B}$ & 4.2 & $\mathrm{~F}$ & $\mathrm{~T}$ & 2 & Tr, Ner & 38 & 7660 \\
\hline A. longipes Agardh & $\mathrm{B}$ & 0.3 & $\mathrm{~F}$ & $\mathrm{P}$ & 2 & $\mathrm{Tr}, \mathrm{Tm}, \mathrm{Ner}$ & & 10 \\
\hline A. sp. & $\mathrm{B}$ & 3.0 & $\mathrm{~F}$ & $\mathrm{~T}$ & 2 & Ner & 89 & 5061 \\
\hline $\begin{array}{l}\text { Actinocyclus octonarius Ehrenberg } \\
\text { var. octonarius }\end{array}$ & $\mathrm{R}, \mathrm{B}$ & 33.6 & $\mathrm{~F}$ & $\mathrm{P}$ & 2 & Ner, Oc & 25 & 3220 \\
\hline $\begin{array}{l}\text { A. octonarius var. ralfsii (W. Smith) } \\
\text { Hustedt }\end{array}$ & $\mathrm{R}$ & 1.5 & $\mathrm{~F}$ & $\mathrm{P}$ & 2 & Ner & & \\
\hline $\begin{array}{l}\text { A. octonarius var. tenellus } \\
\text { (Brébisson) ex Villareal et Fryxell }\end{array}$ & $\mathrm{R}, \mathrm{B}$ & 1.8 & $\mathrm{~F}$ & $\mathrm{P}$ & 2 & Ner & 16 & 36 \\
\hline A. subtilis (Gregory) Ralfs & $\mathrm{R}$ & 1.8 & $\mathrm{~F}$ & $\mathrm{P}$ & 2 & Ner & & \\
\hline $\begin{array}{l}\text { Actinoptychus campanulifer A. } \\
\text { Schmidt }\end{array}$ & $\mathrm{R}, \mathrm{B}$ & 1.8 & $\mathrm{~F}$ & $\mathrm{~T}$ & 2 & Tr, Ner & 38 & 88 \\
\hline A. minutus Greville & $\mathrm{R}$ & 0.3 & $\mathrm{~F}$ & $\mathrm{~T}$ & 2 & Sbt, Ner & & \\
\hline A. parvus A. Mann & $\mathrm{B}$ & 0.3 & $\mathrm{~F}$ & $\mathrm{~T}$ & 2 & Sbt, Ner & & 34 \\
\hline A. senarius (Ehrenberg) Ehrenberg & $\mathrm{R}, \mathrm{B}$ & 8.8 & $\mathrm{~F}$ & $P$ & 2 & $\mathrm{Tr}, \mathrm{Tm}, \mathrm{Ner}, \mathrm{Oc}$ & & 1069 \\
\hline A. splendens (Shadbold) Ralfs & $\mathrm{R}$ & 1.2 & $\mathrm{~F}$ & $\mathrm{P}, \mathrm{T}$ & 2 & $\mathrm{Tr}, \mathrm{Tm}, \mathrm{Ner}, \mathrm{Oc}$ & & \\
\hline A. vulgaris Schumann & $\mathrm{R}$ & 0.3 & $\mathrm{~F}$ & $\mathrm{~T}$ & 2 & Sbt, Tm, Ner & & \\
\hline $\begin{array}{l}\text { Alveus marinus (Grunow) } \\
\text { Kaczmarska et Fryxell }\end{array}$ & $\mathrm{R}$ & 0.3 & $\mathrm{~F}$ & $\mathrm{P}$ & 2 & Sbt, Ner & & \\
\hline
\end{tabular}


Apéndice 1. Continuación.

\begin{tabular}{|c|c|c|c|c|c|c|c|c|}
\hline \multirow[b]{2}{*}{ Таха } & \multirow[b]{2}{*}{$\mathrm{M}$} & \multirow[b]{2}{*}{ Frec. } & \multirow[b]{2}{*}{$\mathrm{N}$} & \multirow[b]{2}{*}{$\mathrm{H}$} & \multirow[b]{2}{*}{ NO } & \multirow{2}{*}{$\begin{array}{l}\text { Distribución } \\
\text { Global }\end{array}$} & \multicolumn{2}{|c|}{$\begin{array}{c}\text { Abundancia } \\
\text { céls. } \mathrm{L}^{-1}\end{array}$} \\
\hline & & & & & & & Mín. & Máx. \\
\hline Amphora arenaria Donkin & $\mathrm{R}, \mathrm{B}$ & 8.8 & $\mathrm{~F}$ & $\mathrm{~T}$ & 2 & Tr, Tm, Ner & 36 & 8051 \\
\hline A. decusata Grunow & $\mathrm{R}$ & 1.2 & $\mathrm{~F}$ & $\mathrm{~T}$ & 2 & $\mathrm{Tr}, \mathrm{Oc}$ & & \\
\hline A. ovalis (Kützing) Kützing & B & 0.9 & $\mathrm{~F}$ & $\mathrm{~T}$ & 2 & $\mathrm{Tr}, \mathrm{Ner}$ & 10 & 2323 \\
\hline A. proteus Gregory & B & 3.9 & $\mathrm{~F}$ & $\mathrm{~T}$ & 2 & $\mathrm{Tr}, \mathrm{Tm}, \mathrm{Ner}$ & 25 & 2195 \\
\hline A. veneta Kützing & B & 0.3 & $\mathrm{~F}$ & $\mathrm{~T}$ & 2 & Sbt, Ner & & 352 \\
\hline A. cf. egregia Ehrenberg & $\mathrm{R}$ & 3.9 & $\mathrm{~F}$ & $\mathrm{~T}$ & 2 & Tr, Ner & & \\
\hline $\begin{array}{l}\text { Asterionellopsis glacialis } \\
\text { (Castracane) Round }\end{array}$ & $\mathrm{R}, \mathrm{B}$ & 16.7 & $\mathrm{~F}$ & $\mathrm{P}$ & 4 & Fr, Tm, Ner & 193 & 44278 \\
\hline Asteromphalus elegans Greville & B & 1.2 & $\mathrm{~F}$ & $\mathrm{~T}$ & 2 & Tr, Ner, Oc & 35 & 53 \\
\hline A. flabellatus (Brébisson) Greville & B & 0.9 & $\mathrm{~F}$ & $\mathrm{~T}$ & 2 & Tr, Sbt, Ner, Oc & 32 & 49 \\
\hline A. heptactis (Brébisson) Ralfs & $\mathrm{R}, \mathrm{B}$ & 14.2 & $\mathrm{~F}$ & $\mathrm{P}$ & 2 & $\mathrm{Tr}, \mathrm{Tm}, \mathrm{Ner}$ & 13 & 3809 \\
\hline $\begin{array}{l}\text { A. petterssonii (Kolbe) Thorrington- } \\
\text { Smith }\end{array}$ & $\mathrm{B}$ & 2.4 & $\mathrm{~F}$ & $\mathrm{P}$ & 2 & $\mathrm{Tr}, \mathrm{Sbt}$ & 20 & 1960 \\
\hline A. robustus Castracane & $\mathrm{B}$ & 3.6 & $\mathrm{~F}$ & $\mathrm{~T}$ & 2 & $\mathrm{Tr}, \mathrm{Sbt}$ & 25 & 121 \\
\hline A. vanheurckii A. Mann & B & 0.6 & $\mathrm{~F}$ & $\mathrm{P}$ & 2 & $\mathrm{Tr}, \mathrm{Sbt}, \mathrm{Ner}$ & 35 & 52 \\
\hline $\begin{array}{l}\text { Aulacoseira granulata (Ehrenberg) } \\
\text { Simonsen }\end{array}$ & $\mathrm{R}$ & 0.3 & $\mathrm{~F}$ & $\mathrm{P}, \mathrm{T}$ & 2 & Slb & & \\
\hline $\begin{array}{l}\text { Azpeitia nodulifera (A. Schmidt) } \\
\text { Fryxell et Sims }\end{array}$ & $\mathrm{R}, \mathrm{B}$ & 15.5 & $\mathrm{~F}$ & $\mathrm{~T}$ & 2 & Tr, Ner & 24 & 2829 \\
\hline Bacillaria paxillifer (Müller) Hendey & $\mathrm{R}$ & 0.9 & $\mathrm{~F}$ & $\mathrm{P}$ & 4 & Ner? & & \\
\hline Bacteriastrum comosum Pavillard & $\mathrm{R}$ & 0.3 & $\mathrm{~F}$ & $\mathrm{P}$ & 4 & Tr, Ner & & \\
\hline B. delicatulum Cleve & $\mathrm{R}, \mathrm{B}$ & 20.3 & $\mathrm{~F}$ & $\mathrm{P}$ & 4 & Tr, Tm, Ner, Oc & 53 & 51635 \\
\hline B. elongatum Cleve & $\mathrm{B}$ & 2.4 & $\mathrm{~F}$ & $\mathrm{P}$ & 4 & $\mathrm{Tr}, \mathrm{Tm}, \mathrm{Oc}$ & 160 & 4268 \\
\hline B. furcatum Shadbold & $\mathrm{R}, \mathrm{B}$ & 10.3 & $\mathrm{~F}$ & $\mathrm{P}$ & 4 & $\mathrm{Tr}, \mathrm{Tm}, \mathrm{Oc}$ & 42 & 31017 \\
\hline B. hyalinum Lauder & $\mathrm{R}, \mathrm{B}$ & 14.6 & $\mathrm{~F}$ & $\mathrm{P}$ & 4 & $\mathrm{Tr}, \mathrm{Tm}, \mathrm{Ner}, \mathrm{Oc}$ & 43 & 10670 \\
\hline Bellerochea horologicalis von Stosch & $\mathrm{R}, \mathrm{B}$ & 1.8 & $\mathrm{~F}$ & $\mathrm{P}$ & 4 & Tm, Ner & 200 & 543 \\
\hline $\begin{array}{l}\text { B. malleus (Brightwell) van Heurck } \\
\text { emend. von Stosch }\end{array}$ & $\mathrm{B}$ & 2.7 & $\mathrm{~F}$ & $\mathrm{P}$ & 4 & $\mathrm{Tr}, \mathrm{Tm}, \mathrm{Oc}$ & 288 & 2517 \\
\hline $\begin{array}{l}\text { Biddulphia alternas (Bailey) van } \\
\text { Heurck }\end{array}$ & $\mathrm{R}, \mathrm{B}$ & 2.4 & $\mathrm{~F}$ & $\mathrm{P}$ & 2 & Tr, Sbt, Ner, Oc & 34 & 2647 \\
\hline B. biddulphiana (J.E. Smith) Boyer & $\mathrm{R}, \mathrm{B}$ & 3.9 & $\mathrm{~F}$ & $\mathrm{~T}$ & 2 & $\mathrm{Tr}, \mathrm{Tm}, \mathrm{Ner}$ & 21 & 721 \\
\hline B. tridens (Ehrenberg) Ehrenberg & $\mathrm{R}, \mathrm{B}$ & 3.0 & $\mathrm{~F}$ & $\mathrm{P}$ & 4 & $\mathrm{Tr}, \mathrm{Tm}, \mathrm{Ner}$ & 34 & 2647 \\
\hline Campylodiscus decorus H. Peragallo & $\mathrm{R}$ & 0.3 & $\mathrm{~F}$ & $\mathrm{~T}$ & 2 & Tr, Ner & & \\
\hline Campylosira sp. & B & 0.3 & $\mathrm{~F}$ & $\mathrm{~T}$ & 2 & $\mathrm{Tr}, \mathrm{Ner}$ & & 7001 \\
\hline $\begin{array}{l}\text { Cerataulina pelagica (Cleve) } \\
\text { Hendey* }\end{array}$ & $\mathrm{R}, \mathrm{B}$ & 46.6 & $\mathrm{~F}$ & $\mathrm{P}$ & 4 & $\mathrm{Tr}, \mathrm{Oc}$ & 10 & 21712 \\
\hline Cerataulus californianus A. Schmidt & $\mathrm{R}$ & 0.6 & $\mathrm{~F}$ & $\mathrm{~T}$ & 2 & Sbt, Ner & & \\
\hline Chaetoceros aequatorialis Cleve & $\mathrm{R}$ & 0.3 & $\mathrm{~F}$ & $P$ & 4 & $\mathrm{Tr}, \mathrm{Oc}$ & & \\
\hline
\end{tabular}


Apéndice 1. Continuación.

\begin{tabular}{|c|c|c|c|c|c|c|c|c|}
\hline \multirow[b]{2}{*}{ Taxa } & \multirow[b]{2}{*}{$\mathrm{M}$} & \multirow[b]{2}{*}{ Frec. } & \multirow[b]{2}{*}{$\mathrm{N}$} & \multirow[b]{2}{*}{$\mathrm{H}$} & \multirow[b]{2}{*}{ NO } & \multirow{2}{*}{$\begin{array}{l}\text { Distribución } \\
\text { Global }\end{array}$} & \multicolumn{2}{|c|}{$\begin{array}{l}\text { Abundancia } \\
\text { céls. } \mathrm{L}^{-1}\end{array}$} \\
\hline & & & & & & & Mín. & Máx. \\
\hline C. affinis Lauder var. affinis & $\mathrm{R}, \mathrm{B}$ & 46.4 & $\mathrm{~F}$ & $\mathrm{P}$ & 4 & Ner, Oc & 16 & 30671 \\
\hline $\begin{array}{l}\text { C. affinis var. circinalis (Meunier) } \\
\text { Hustedt }\end{array}$ & $\mathrm{R}$ & 0.3 & $\mathrm{~F}$ & $\mathrm{P}$ & 4 & Ner & & \\
\hline C. affinis var. willei (Gran) Hustedt & $\mathrm{R}, \mathrm{B}$ & 3.3 & $\mathrm{~F}$ & $\mathrm{P}$ & 4 & Ner & 323 & 9042 \\
\hline C. anastomosans Grunow & B & 0.9 & $\mathrm{~F}$ & $\mathrm{P}$ & 4 & $\mathrm{Tr}, \mathrm{Oc}$ & 9469 & 14661 \\
\hline C. atlanticus Cleve var. atlanticus & $\mathrm{R}, \mathrm{B}$ & 2.1 & $\mathrm{~F}$ & $\mathrm{P}$ & 4 & Ner, Oc & 160 & 2243 \\
\hline $\begin{array}{l}\text { C. atlanticus var. neapolitanus } \\
\text { (Schröder) Hustedt }\end{array}$ & $\mathrm{R}$ & 0.3 & $\mathrm{~F}$ & $\mathrm{P}$ & 4 & Sbt, Tm, Ner & & \\
\hline $\begin{array}{l}\text { C. atlanticus var. skeleton (Schütt) } \\
\text { Hustedt }\end{array}$ & $\mathrm{R}$ & 0.9 & $\mathrm{~F}$ & $\mathrm{P}$ & 4 & $\operatorname{Tr}, \mathrm{Tm}$ & & \\
\hline C. borealis Bailey & $\mathrm{R}$ & 0.3 & $\mathrm{~F}$ & $\mathrm{P}$ & 4 & Tm, Fr, Ner? & & \\
\hline C. brevis Schütt & $\mathrm{R}, \mathrm{B}$ & 25.8 & $\mathrm{~F}$ & $\mathrm{P}$ & 4 & Tr, Tm, Ner, Oc & 911 & 103109 \\
\hline C. coarctatus Lauder & $\mathrm{R}, \mathrm{B}$ & 26.4 & $\mathrm{~F}$ & $\mathrm{P}$ & 4 & $\mathrm{Tr}, \mathrm{Oc}$ & 25 & 38893 \\
\hline C. compressus Lauder & $\mathrm{R}, \mathrm{B}$ & 13.0 & $\mathrm{~F}$ & $\mathrm{P}$ & 4 & $\operatorname{Tr}, \mathrm{Tm}$ & 108 & 59804 \\
\hline C. convolutus Castracane* & $\mathrm{R}$ & 0.9 & $\mathrm{~F}$ & $\mathrm{P}$ & 4 & Ner, Oc & & \\
\hline C. curvisetus Castracane* & $\mathrm{R}, \mathrm{B}$ & 90.6 & $\mathrm{~F}$ & $\mathrm{P}$ & 4 & Ner, Oc & 30 & 660725 \\
\hline C. dadayi Pavillard & $\mathrm{R}, \mathrm{B}$ & 1.2 & $\mathrm{~F}$ & $\mathrm{P}$ & 4 & $\mathrm{Tr}, \mathrm{Oc}$ & 60 & 356 \\
\hline C. danicus Cleve & $\mathrm{R}, \mathrm{B}$ & 10.6 & $\mathrm{~F}$ & $\mathrm{P}$ & 4 & Ner, Oc & 20 & 6984 \\
\hline C. debilis Cleve* & $\mathrm{R}, \mathrm{B}$ & 5.2 & $\mathrm{~F}$ & $\mathrm{P}$ & 4 & Ner, Oc & 50 & 17783 \\
\hline C. decipiens Cleve & $\mathrm{R}, \mathrm{B}$ & 52.7 & $\mathrm{~F}$ & $\mathrm{P}$ & 4 & Ner, Oc & 30 & 82347 \\
\hline C. densus (Cleve) Cleve & $\mathrm{R}$ & 1.2 & $\mathrm{~F}$ & $\mathrm{P}$ & 4 & Ner, Oc & & \\
\hline C. dichaeta Ehrenberg & $\mathrm{B}$ & 2.7 & $\mathrm{~F}$ & $\mathrm{P}$ & 4 & $\mathrm{Tm}, \mathrm{Tr}, \mathrm{Oc}$ & 96 & 8389 \\
\hline C. didymus Ehrenberg var. didymus & $\mathrm{R}, \mathrm{B}$ & 25.2 & $\mathrm{~F}$ & $\mathrm{P}$ & 4 & Tr, Sbt, Ner, Oc & 40 & 22397 \\
\hline $\begin{array}{l}\text { C. didymus var. anglicus (Grunow) } \\
\text { Gran }\end{array}$ & $\mathrm{R}, \mathrm{B}$ & 0.9 & $\mathrm{~F}$ & $\mathrm{P}$ & 4 & Tr, Ner & 502 & 739 \\
\hline C. difficilis Cleve & $\mathrm{R}$ & 1.5 & $\mathrm{~F}$ & $\mathrm{P}$ & 4 & $\mathrm{Tr}, \mathrm{Sbt}, \mathrm{Oc}$ & & \\
\hline C. diversus Cleve & $\mathrm{R}, \mathrm{B}$ & 25.5 & $\mathrm{~F}$ & $\mathrm{P}$ & 4 & $\mathrm{Tr}, \mathrm{Oc}$ & 100 & 37771 \\
\hline C. eibenii Grunow & $\mathrm{R}, \mathrm{B}$ & 6.0 & $\mathrm{~F}$ & $\mathrm{P}$ & 4 & $\mathrm{Tr}, \mathrm{Tm}, \mathrm{Oc}$ & 86 & 13695 \\
\hline C. laciniosus Schütt & $\mathrm{R}, \mathrm{B}$ & 52.7 & $\mathrm{~F}$ & $\mathrm{P}$ & 4 & $\operatorname{Tr}, \mathrm{Tm}, \mathrm{Ner}, \mathrm{Oc}$ & 40 & 276449 \\
\hline C. lauderi Ralfs & $\mathrm{R}, \mathrm{B}$ & 0.9 & $\mathrm{~F}$ & $\mathrm{P}$ & 4 & Tr, Tm, Ner, Oc & 361 & 20630 \\
\hline C. lorenzianus Grunow & $\mathrm{R}, \mathrm{B}$ & 24.6 & $\mathrm{~F}$ & $\mathrm{P}$ & 4 & Tr, Ner, Oc & 60 & 91354 \\
\hline C. messanensis Castracane & $\mathrm{R}, \mathrm{B}$ & 10.0 & $\mathrm{~F}$ & $\mathrm{P}$ & 4 & $\mathrm{Tr}, \mathrm{Oc}$ & 40 & 35747 \\
\hline C. mitra (Bailey) Cleve & $\mathrm{R}$ & 0.6 & $\mathrm{~F}$ & $\mathrm{P}$ & 4 & $\mathrm{Tm}, \mathrm{Oc}$ & & \\
\hline C. pendulus Karsten & $\mathrm{R}, \mathrm{B}$ & 1.2 & $\mathrm{~F}$ & $\mathrm{P}$ & 2 & $\mathrm{Tr}, \mathrm{Sbt}, \mathrm{Oc}$ & 20 & 58 \\
\hline C. perpusillus Cleve & $\mathrm{R}, \mathrm{B}$ & 18.8 & $\mathrm{~F}$ & $\mathrm{P}$ & 4 & Sbt, Tm, Oc & 132 & 107350 \\
\hline C. peruvianus Brightwell & $\mathrm{R}, \mathrm{B}$ & 55.2 & $\mathrm{~F}$ & $\mathrm{P}$ & 2 & $\mathrm{Tr}, \mathrm{Tm}, \mathrm{Ner}, \mathrm{Oc}$ & 20 & 9919 \\
\hline C. protuberans Lauder & $\mathrm{R}$ & 2.4 & $\mathrm{~F}$ & $\mathrm{P}$ & 4 & Tr, Ner & & \\
\hline C. pseudoaurivilli Ikari & $\mathrm{R}$ & 2.4 & $\mathrm{~F}$ & $\mathrm{P}$ & 4 & Tr, Ner & & \\
\hline
\end{tabular}


Apéndice 1. Continuación.

\begin{tabular}{|c|c|c|c|c|c|c|c|c|}
\hline \multirow[b]{2}{*}{ Taxa } & \multirow[b]{2}{*}{ M } & \multirow[b]{2}{*}{ Frec. } & \multirow[b]{2}{*}{$\mathrm{N}$} & \multirow[b]{2}{*}{$\mathrm{H}$} & \multirow[b]{2}{*}{$\mathrm{NO}$} & \multirow{2}{*}{$\begin{array}{l}\text { Distribución } \\
\text { Global }\end{array}$} & \multicolumn{2}{|c|}{$\begin{array}{l}\text { Abundancia } \\
\text { céls. } \mathrm{L}^{-1}\end{array}$} \\
\hline & & & & & & & Mín. & Máx. \\
\hline C. pseudocurvisetus Manguin & $\mathrm{R}$ & 0.3 & $\mathrm{~F}$ & $\mathrm{P}$ & 4 & $\mathrm{Tr}, \mathrm{Oc}$ & & \\
\hline C. radicans Schütt & $\mathrm{R}, \mathrm{B}$ & 18.8 & $\mathrm{~F}$ & $\mathrm{P}$ & 4 & Ner, Oc & 715 & 266675 \\
\hline C. rostratus Lauder & $\mathrm{R}$ & 1.5 & $\mathrm{~F}$ & $\mathrm{P}$ & 4 & $\mathrm{Tr}, \mathrm{Oc}$ & & \\
\hline C. simplex Ostenfeld & B & 9.4 & $\mathrm{~F}$ & $\mathrm{P}$ & 2 & $\mathrm{Tm}$ & 89 & 5061 \\
\hline C. socialis Lauder* & $\mathrm{R}, \mathrm{B}$ & 21.8 & $\mathrm{~F}$ & $\mathrm{P}$ & 4 & Ner, Oc & 467 & 60473 \\
\hline C. tenuissimus Meunier & $\mathrm{B}$ & 1.2 & $\mathrm{~F}$ & $\mathrm{P}$ & 4 & $\mathrm{Tm}$ & 193 & 12803 \\
\hline C. teres Cleve & $\mathrm{R}, \mathrm{B}$ & 4.2 & $\mathrm{~F}$ & $\mathrm{P}$ & 4 & Tr, Tm, Ner, Oc & 285 & 31275 \\
\hline C. tetrastichon Cleve & $\mathrm{R}, \mathrm{B}$ & 0.6 & $\mathrm{~F}$ & $\mathrm{P}$ & 4 & $\mathrm{Tr}, \mathrm{Oc}$ & 203 & 155771 \\
\hline C. tortissimus Gran & $\mathrm{R}, \mathrm{B}$ & 13.9 & $\mathrm{~F}$ & $\mathrm{P}$ & 4 & Ner, Oc & 1073 & 364678 \\
\hline C. wighamii Brightwell* & $\mathrm{R}, \mathrm{B}$ & 1.5 & $\mathrm{~F}$ & $\mathrm{P}$ & 4 & $\mathrm{Tr}, \mathrm{Oc}, \mathrm{Slb}$ & 89 & 12590 \\
\hline C. sp. 1 & $\mathrm{~B}$ & 20.3 & $\mathrm{~F}$ & $\mathrm{P}$ & 3 & Tr, Ner, & 200 & 559200 \\
\hline C. sp. 2 & $\mathrm{~B}$ & 13.9 & $\mathrm{~F}$ & $\mathrm{P}$ & 2 & Tr, Ner, & 40 & 156885 \\
\hline C.sp. 3 & $\mathrm{R}$ & 2.7 & $\mathrm{~F}$ & $\mathrm{P}$ & 4 & $\mathrm{Tr}, \mathrm{Sbt}$ & 178 & 3500 \\
\hline $\begin{array}{l}\text { Climacodium frauenfeldianum } \\
\text { Grunow }\end{array}$ & $\mathrm{R}, \mathrm{B}$ & 18.5 & $\mathrm{~F}$ & $\mathrm{P}$ & 4 & $\mathrm{Tr}, \mathrm{Oc}$ & 43 & 7989 \\
\hline Climacosphenia moniligera Ehrenberg & $\mathrm{R}$ & 0.3 & $\mathrm{~F}$ & $\mathrm{P}$ & 2 & $\mathrm{Tr}, \mathrm{Ner}, \mathrm{Oc}$ & & \\
\hline Cocconeis convexa Giffen & $\mathrm{B}$ & 0.3 & $\mathrm{~F}$ & $\mathrm{~T}$ & 2 & Sbt, Ner & 10 & 533 \\
\hline C. distans Gregory & $\mathrm{R}$ & 3.3 & $\mathrm{~F}$ & $\mathrm{~T}$ & 2 & Tr, Sbt, Ner & & \\
\hline C. molesta var. crucifera Grunow & B & 0.3 & $\mathrm{~F}$ & $\mathrm{~T}$ & 2 & Tr, Ner & & 10 \\
\hline C. placentula Ehrenberg & $\mathrm{B}$ & 0.3 & $\mathrm{~F}$ & $\mathrm{~T}$ & 2 & Tr, Ner & & 86 \\
\hline C. scutellum Ehrenberg & $\mathrm{R}, \mathrm{B}$ & 2.1 & $\mathrm{~F}$ & $\mathrm{~T}$ & 2 & $\mathrm{Tr}, \mathrm{Tm}, \mathrm{Slb}$ & 27 & 3066 \\
\hline C. sp. & $\mathrm{B}$ & 3.3 & $\mathrm{~F}$ & $\mathrm{~T}$ & 2 & Tr, Ner & 58 & 215 \\
\hline Corethron hystrix Hensen & $\mathrm{B}$ & 12.1 & $\mathrm{~F}$ & $\mathrm{P}$ & 2 & Ner, Oc & 20 & 7619 \\
\hline $\begin{array}{l}\text { Coscinodiscus asteromphalus } \\
\text { Ehrenberg }\end{array}$ & $\mathrm{R}$ & 3.0 & $\mathrm{~F}$ & $\mathrm{~T}$ & 2 & Tr, Sbt, Ner & & \\
\hline C. centralis Ehrenberg* & $\mathrm{R}, \mathrm{B}$ & 7.3 & $\mathrm{~F}$ & $\mathrm{P}$ & 2 & Sbt, Tm, Ner, Oc & 29 & 739 \\
\hline C. concinnus W. Smith* & $\mathrm{R}, \mathrm{B}$ & 3.3 & $\mathrm{~F}$ & $\mathrm{P}$ & 2 & Tr, Sbt, Ner, Oc & 27 & 82 \\
\hline C. gigas Ehrenberg & $\mathrm{R}, \mathrm{B}$ & 6.7 & $\mathrm{~F}$ & $\mathrm{P}, \mathrm{T}$ & 2 & $\mathrm{Tr}, \mathrm{Sbt}$ & 25 & 125 \\
\hline C. granii Gough & $\mathrm{R}, \mathrm{B}$ & 35.8 & $\mathrm{~F}$ & $\mathrm{P}$ & 2 & Sbt, Tm, Ner, Oc & 10 & 1704 \\
\hline C. radiatus Gough & $\mathrm{R}, \mathrm{B}$ & 46.0 & $\mathrm{~F}$ & $\mathrm{P}$ & 2 & Tr, Sbt, Ner, Oc & 10 & 4102 \\
\hline C. wailesii Gran et Angst* & $\mathrm{R}$ & 0.6 & $\mathrm{~F}$ & $\mathrm{P}$ & 2 & $\mathrm{Tr}, \mathrm{Tm}, \mathrm{Oc}$ & & \\
\hline C. sp. & $\mathrm{B}$ & 12.1 & $\mathrm{~F}$ & $\mathrm{P}$ & 2 & Tr, Ner & 20 & 1960 \\
\hline $\begin{array}{l}\text { Cyclotella littoralis Lange et } \\
\text { Syvertsen }\end{array}$ & B & 0.3 & $\mathrm{~F}$ & $\mathrm{P}$ & 2 & $\begin{array}{c}\text { Tm, HNyS, Ner, } \\
\text { Oc }\end{array}$ & & 84 \\
\hline C. stylorum Brightwell & B & 0.3 & $\mathrm{~F}$ & $\mathrm{P}$ & 2 & Tr, Tm, HS, Ner & & 251 \\
\hline $\begin{array}{l}\text { Cylindrotheca closterium } \\
\text { (Ehrenberg) Reiman et Lewin* }\end{array}$ & $\mathrm{R}, \mathrm{B}$ & 82.4 & $\mathrm{~F}$ & $\mathrm{~T}$ & 2 & Ner, Oc & 80 & 61282 \\
\hline Cymatonitzschia sp. & $\mathrm{B}$ & 0.3 & $\mathrm{~F}$ & $\mathrm{~T}$ & 2 & $\mathrm{Tm}, \operatorname{Tr}$ & & 20 \\
\hline
\end{tabular}


Apéndice 1. Continuación.

\begin{tabular}{|c|c|c|c|c|c|c|c|c|}
\hline \multirow[b]{2}{*}{ Taxa } & \multirow[b]{2}{*}{ M } & \multirow[b]{2}{*}{ Frec. } & \multirow[b]{2}{*}{$\mathrm{N}$} & \multirow[b]{2}{*}{$\mathrm{H}$} & \multirow[b]{2}{*}{$\mathrm{NO}$} & \multirow{2}{*}{$\begin{array}{l}\text { Distribución } \\
\text { Global }\end{array}$} & \multicolumn{2}{|c|}{$\begin{array}{l}\text { Abundancia } \\
\text { céls. } \mathrm{L}^{-1}\end{array}$} \\
\hline & & & & & & & Mín. & Máx. \\
\hline Cymatosira lorenziana Grunow & $\mathrm{R}, \mathrm{B}$ & 1.5 & $\mathrm{~F}$ & $\mathrm{P}$ & 4 & $\mathrm{Tr}, \mathrm{Tm}, \mathrm{Ner}, \mathrm{Oc}$ & 20 & 1854 \\
\hline $\begin{array}{l}\text { Dactyliosolen fragilissimus (Bergon) } \\
\text { Hasle }\end{array}$ & $\mathrm{R}, \mathrm{B}$ & 59.7 & $\mathrm{~F}$ & $\mathrm{P}$ & 4 & Ner, Oc & 80 & 72351 \\
\hline D. phuketensis (Sundström) Hasle & $\mathrm{R}, \mathrm{B}$ & 47.9 & $\mathrm{~F}$ & $\mathrm{P}$ & 4 & $\mathrm{Tr}, \mathrm{Tm}$ & 40 & 100593 \\
\hline $\begin{array}{l}\text { Delphineis surirella var. australis } \\
\text { (Petit) Tsarenko }\end{array}$ & B & 16.4 & $\mathrm{~F}$ & $\mathrm{P}$ & 2 & Sbt, Tm, Ner & 20 & 21728 \\
\hline D. minutissima (Hustedt) Simonsen & B & 0.3 & $\mathrm{~F}$ & $\mathrm{~T}$ & 2 & Tr, Tm, Ner & & 158 \\
\hline Detonula pumila (Castracane) Gran & $\mathrm{R}, \mathrm{B}$ & 30.6 & $\mathrm{~F}$ & $\mathrm{P}$ & 4 & $\mathrm{Tr}, \mathrm{Tm}, \mathrm{Ner}$ & 54 & 35291 \\
\hline $\begin{array}{l}\text { Diploneis campylodiscus (Grunow) } \\
\text { Cleve }\end{array}$ & B & 0.3 & $\mathrm{~F}$ & $\mathrm{~T}$ & 2 & Tm, Tr, Ner & & 13 \\
\hline D. cf. chersonensis (Grunow) Cleve & $\mathrm{R}$ & 0.3 & $\mathrm{~F}$ & $\mathrm{~T}$ & 2 & Ner, polihalina & & 1041 \\
\hline D. crabro (Ehrenberg) Ehrenberg & $\mathrm{R}$ & 0.3 & $\mathrm{~F}$ & $\mathrm{~T}$ & 2 & Ner & & 47 \\
\hline D. gemmata Ehrenberg & $\mathrm{R}$ & 0.3 & $\mathrm{~F}$ & $\mathrm{~T}$ & 2 & Tr, Ner & & \\
\hline D. weissflogii (A. Schmidt) Cleve & B & 0.9 & $\mathrm{~F}$ & $\mathrm{~T}, \mathrm{P}$ & 2 & $\mathrm{Tr}, \mathrm{Sbt}, \mathrm{Ner}, \mathrm{Oc}$ & 20 & 1295 \\
\hline D. cf. smithii (Brébisson) Cleve & B & 0.3 & $\mathrm{~F}$ & $\mathrm{~T}$ & 2 & Tr, Ner & & 246 \\
\hline D. sp. & B & 0.3 & $\mathrm{~F}$ & $\mathrm{~T}$ & 2 & Tr, Ner & & 15 \\
\hline Ditylum brightwellii (West) Grunow* & $\mathrm{R}$ & 53.3 & $\mathrm{~F}$ & $\mathrm{P}$ & 2 & Ner, Oc & 13 & 11112 \\
\hline Entomoneis alata (West) Grunow & B & 0.3 & $\mathrm{~F}$ & $\mathrm{P}$ & 2 & $\mathrm{Slb}, \mathrm{Oc}$ & & 2197 \\
\hline E. gigantea (Grunow) Nizamuddin & B & 0.3 & $\mathrm{~F}$ & $\mathrm{P}$ & 2 & $\mathrm{Slb}, \mathrm{Oc}$ & & 25 \\
\hline Eucampia cornuta (Cleve) Grunow & $\mathrm{R}, \mathrm{B}$ & 38.2 & $\mathrm{~F}$ & $P$ & 4 & $\mathrm{Tr}, \mathrm{Oc}$ & 20 & 117638 \\
\hline E. zodiacus Ehrenberg & $\mathrm{R}, \mathrm{B}$ & 3.3 & $\mathrm{~F}$ & $P$ & 4 & $\mathrm{Tr}, \mathrm{Tm}, \mathrm{Oc}$ & 51 & 320 \\
\hline Eupodiscus radiatus Bailey & $\mathrm{R}$ & 0.3 & $\mathrm{~F}$ & $\mathrm{P}$ & 2 & Sbt, Tm, Ner, Oc & & \\
\hline Fragilaria sp. & B & 3.0 & $\mathrm{~F}$ & $\mathrm{~T}$ & 4 & $\mathrm{Tm}, \mathrm{Tr}, \mathrm{Ner}$ & 41 & 4738 \\
\hline $\begin{array}{l}\text { Fragilariopsis doliolus (Wallich) } \\
\text { Medlin et Sims }\end{array}$ & $\mathrm{R}, \mathrm{B}$ & 7.3 & $\mathrm{~F}$ & $\mathrm{P}$ & 4 & Tr, Ner & 40 & 3013 \\
\hline Gossleriella tropica Schütt & $\mathrm{R}$ & 0.3 & $\mathrm{~F}$ & $\mathrm{P}$ & 2 & $\mathrm{Tr}, \mathrm{Oc}, \mathrm{Umb}$ & & \\
\hline Grammatophora angulosa Ehrenberg & $\mathrm{R}, \mathrm{B}$ & 0.9 & $\mathrm{~F}$ & $\mathrm{~T}$ & 4 & Tr, Ner & 242 & 557 \\
\hline G. marina (Lyngbye) Kützing & $\mathrm{B}$ & 0.6 & $\mathrm{~F}$ & $\mathrm{~T}$ & 4 & Sbt, Tm, Ner & 20 & 60 \\
\hline Guinardia cylindrus (Cleve) Hasle & $\mathrm{R}, \mathrm{B}$ & 3.3 & $\mathrm{~F}$ & $\mathrm{P}$ & 4 & Tr, Ner, Oc & 13 & 221 \\
\hline G. delicatula (Cleve) Hasle & $\mathrm{B}$ & 42.7 & $\mathrm{~F}$ & $\mathrm{P}$ & 4 & $\mathrm{Tm}, \mathrm{Tr}, \mathrm{Ner}, \mathrm{Oc}$ & 58 & 100593 \\
\hline G. flaccida (Castracane) H. Peragallo & $\mathrm{R}, \mathrm{B}$ & 34.9 & $\mathrm{~F}$ & $\mathrm{P}$ & 4 & Tr, Tm, Ner, Oc & 10 & 22824 \\
\hline G. striata (Stolterfoth) Hasle & $\mathrm{R}, \mathrm{B}$ & 67.6 & $\mathrm{~F}$ & $\mathrm{P}$ & 4 & Tr, Ner, Oc & 46 & 127102 \\
\hline $\begin{array}{l}\text { Gyrosigma balticum (Ehrenberg) } \\
\text { Rabenhorst }\end{array}$ & $\mathrm{R}, \mathrm{B}$ & 8.5 & $\mathrm{~F}$ & $\mathrm{P}, \mathrm{T}$ & 2 & Tr, Ner, Oc & 20 & 653 \\
\hline G. sp. & $\mathrm{R}$ & 3.3 & $\mathrm{~F}$ & $\mathrm{P}$ & 2 & Tr, Ner & 23 & 80 \\
\hline Hantzschia sp. & B & 0.3 & $\mathrm{~F}$ & $\mathrm{P}$ & 2 & Tr, Ner & & 89 \\
\hline $\begin{array}{l}\text { Haslea frauenfeldii (Grunow) } \\
\text { Simonsen }\end{array}$ & B & 14.6 & $\mathrm{~F}$ & $\mathrm{P}$ & 2 & $\mathrm{Tr}, \mathrm{Oc}$ & 16 & 6856 \\
\hline
\end{tabular}


Apéndice 1. Continuación.

\begin{tabular}{|c|c|c|c|c|c|c|c|c|}
\hline \multirow[b]{2}{*}{ Taxa } & \multirow[b]{2}{*}{ M } & \multirow[b]{2}{*}{ Frec. } & \multirow[b]{2}{*}{$\mathrm{N}$} & \multirow[b]{2}{*}{$\mathrm{H}$} & \multirow[b]{2}{*}{ NO } & \multirow{2}{*}{$\begin{array}{l}\text { Distribución } \\
\text { Global }\end{array}$} & \multicolumn{2}{|c|}{$\begin{array}{c}\text { Abundancia } \\
\text { céls. } \mathrm{L}^{-1} \\
\end{array}$} \\
\hline & & & & & & & Mín. & Máx. \\
\hline H. gigantea (Hustedt) Simonsen & $\mathrm{R}, \mathrm{B}$ & 2.4 & $\mathrm{~F}$ & $\mathrm{P}$ & 2 & $\mathrm{Tr}, \mathrm{Oc}$ & 49 & 2599 \\
\hline H. wawrikae (Hustedt) Simonsen & $\mathrm{R}, \mathrm{B}$ & 31.2 & $\mathrm{~F}$ & $\mathrm{P}$ & 2 & $\mathrm{Tr}, \mathrm{Oc}$ & 25 & 4479 \\
\hline H. sp. & B & 6.4 & $\mathrm{~F}$ & $\mathrm{P}$ & 2 & $\mathrm{Tr}, \mathrm{Ner}$ & 32 & 2194 \\
\hline $\begin{array}{l}\text { Helicotheca tamesis (Shrubsole) } \\
\text { Ricard }\end{array}$ & $\mathrm{R}, \mathrm{B}$ & 54.9 & $\mathrm{~F}$ & $\mathrm{P}$ & 4 & $\mathrm{Tr}, \mathrm{Tm}, \mathrm{Oc}$ & 10 & 12584 \\
\hline $\begin{array}{l}\text { Hemiaulus hauckii Grunow et Van } \\
\text { Heurck }\end{array}$ & $\mathrm{R}, \mathrm{B}$ & 51.8 & $\mathrm{~F}$ & $\mathrm{P}$ & 4 & $\mathrm{Tr}, \mathrm{Tm}, \mathrm{Ner}, \mathrm{Oc}$ & 20 & 55994 \\
\hline H. membranaceus Cleve & $\mathrm{R}, \mathrm{B}$ & 28.8 & $\mathrm{~F}$ & $\mathrm{P}$ & 4 & Tr, Ner, Oc & 48 & 19396 \\
\hline H. sinensis Greville & $\mathrm{R}, \mathrm{B}$ & 6.7 & $\mathrm{~F}$ & $\mathrm{P}$ & 4 & Tr, Tm, Ner, Oc & 100 & 464 \\
\hline Hemidiscus cuneiformis Wallich & $\mathrm{R}, \mathrm{B}$ & 2.7 & $\mathrm{~F}$ & $\mathrm{P}$ & 2 & Tr, Ner & 18 & 56 \\
\hline H. orbicularis (Castracane) Kuntze & $\mathrm{R}, \mathrm{B}$ & 0.9 & $\mathrm{~F}$ & $\mathrm{P}$ & 2 & $\operatorname{Tr}, \operatorname{Tm}$ & 15 & 35 \\
\hline H. ventricosus (Castracane) Mann & $\mathrm{R}$ & 0.9 & $\mathrm{~F}$ & $\mathrm{P}$ & 2 & Sbt, Ner & & \\
\hline Isthmia nervosa Kützing & $\mathrm{R}$ & 0.3 & $\mathrm{~F}$ & $\mathrm{~T}$ & 2 & Sbt, Tm, Ner & & \\
\hline Lauderia annulata Cleve & $\mathrm{R}, \mathrm{B}$ & 10.6 & $\mathrm{~F}$ & $\mathrm{P}$ & 2 & $\mathrm{Tr}, \mathrm{Tm}, \mathrm{Ner}, \mathrm{Oc}$ & 20 & 2691 \\
\hline Leptocylindrus danicus Cleve & $\mathrm{R}, \mathrm{B}$ & 83.6 & $\mathrm{~F}$ & $\mathrm{P}$ & 4 & $\begin{array}{l}\text { Cos, no Ant., } \\
\text { Ner, Oc }\end{array}$ & 160 & 145492 \\
\hline $\begin{array}{l}\text { L. mediterraneus (H. Peragallo) Hasle } \\
\text { (consorcio Solenicola setigera-L. } \\
\text { mediterraneus) }\end{array}$ & $\mathrm{R}, \mathrm{B}$ & 21.2 & $\mathrm{~F}$ & $\mathrm{P}$ & 4 & Ner, Oc & 20 & 6984 \\
\hline L. minimus Gran* & $\mathrm{R}, \mathrm{B}$ & 38.2 & $\mathrm{~F}$ & $\mathrm{P}$ & 4 & $\begin{array}{l}\text { Cos, no Ant., } \\
\text { Ner, Oc }\end{array}$ & 20 & 287439 \\
\hline Licmophora abbreviata Agardh & B & 0.3 & $\mathrm{~F}$ & $\mathrm{~T}$ & 2 & Sbt, Tm, Ner & & 24 \\
\hline L. flabellata (Greville) Agardh & $\mathrm{R}, \mathrm{B}$ & 4.2 & $\mathrm{~F}$ & $\mathrm{~T}$ & 2 & Sbt, Ner & 29 & 2760 \\
\hline L. sp. 1 & B & 0.3 & $\mathrm{~F}$ & $\mathrm{~T}$ & 2 & Ner & & 82 \\
\hline L. sp. 2 & B & 0.9 & $\mathrm{~F}$ & $\mathrm{~T}$ & 2 & Ner & 10 & 154 \\
\hline Lioloma elongatum (Grunow) Hasle & $\mathrm{R}, \mathrm{B}$ & 48.2 & $\mathrm{~F}$ & $\mathrm{~T}, \mathrm{P}$ & 2 & Tr, Ner, Oc & 10 & 8132 \\
\hline L. pacificum (Cupp) Hasle & $\mathrm{R}, \mathrm{B}$ & 29.4 & $\mathrm{~F}$ & $\mathrm{P}$ & 2 & $\operatorname{Tr}, \operatorname{Tm}$ & 25 & 14547 \\
\hline Lithodesmium undulatum Ehrenberg & $\mathrm{R}, \mathrm{B}$ & 30.0 & $\mathrm{~F}$ & $\mathrm{~T}, \mathrm{P}$ & 4 & Tr, Tm, Ner, Oc & 10 & 5032 \\
\hline Lyrella clavata (Gregory) D.G. Mann & B & 0.6 & $\mathrm{~F}$ & $\mathrm{P}$ & 2 & Tr, Tm, Ner & 27 & 49 \\
\hline $\begin{array}{l}\text { L. hennedyii (W. Smith) Stickle et } \\
\text { D.G. Mann }\end{array}$ & B & 0.3 & $\mathrm{~F}$ & $\mathrm{~T}, \mathrm{P}$ & 2 & $\operatorname{Tr}, \mathrm{Tm}, \mathrm{Ner}$ & & 16 \\
\hline L. lyra (Ehrenberg) Karajeva & B & 0.9 & $\mathrm{~F}$ & $\mathrm{P}$ & 2 & $\mathrm{Tr}, \mathrm{Tm}, \mathrm{Ner}$ & 39 & 549 \\
\hline L. sp. & B & 0.3 & $\mathrm{~F}$ & $\mathrm{~T}$ & 2 & Tr, Ner & & 17 \\
\hline Mastogloia binotata (Grunow) Cleve & B & 0.6 & $\mathrm{~F}$ & $\mathrm{~T}$ & 2 & $\operatorname{Tr}, \operatorname{Tm}$ & 82 & 979 \\
\hline M. rostrata (Wallich) Hustedt & B & 0.3 & $\mathrm{~F}$ & $P$ & 2 & $\mathrm{Tr}, \mathrm{Sbt}, \mathrm{Oc}$ & & 66 \\
\hline Melosira numuloides Agardh & B & 1.8 & $\mathrm{~F}$ & $\mathrm{P}$ & 4 & Ner, Oc & 117 & 3920 \\
\hline Meuniera membranacea (Cleve) & $\mathrm{R}$ & 16.7 & $\mathrm{~F}$ & $\mathrm{P}$ & 4 & Tm, Ner, Oc & 56 & 26062 \\
\hline
\end{tabular}


Apéndice 1. Continuación.

\begin{tabular}{|c|c|c|c|c|c|c|c|c|}
\hline \multirow[b]{2}{*}{ Taxa } & \multirow[b]{2}{*}{ M } & \multirow[b]{2}{*}{ Frec. } & \multirow[b]{2}{*}{$\mathrm{N}$} & \multirow[b]{2}{*}{$\mathrm{H}$} & \multirow[b]{2}{*}{ NO } & \multirow{2}{*}{$\begin{array}{l}\text { Distribución } \\
\text { Global }\end{array}$} & \multicolumn{2}{|c|}{$\begin{array}{l}\text { Abundancia } \\
\text { céls. } L^{-1}\end{array}$} \\
\hline & & & & & & & Mín. & Máx. \\
\hline Navicula pennata A. Schmidt & $\mathrm{B}$ & 0.3 & $\mathrm{~F}$ & $P$ & 2 & $\mathrm{Tr}, \mathrm{Tm}$ & & 445 \\
\hline N. sp. 1 & $\mathrm{~B}$ & 19.7 & $\mathrm{~F}$ & $\mathrm{P}$ & 2 & Tr, Ner & 10 & 4102 \\
\hline N. sp. 2 & $\mathrm{~B}$ & 8.8 & $\mathrm{~F}$ & $\mathrm{~T}$ & 2 & Tr, Ner & 20 & 2516 \\
\hline$N$. sp. 3 & $\mathrm{~B}$ & 2.4 & $\mathrm{~F}$ & $\mathrm{~T}$ & 2 & Tr, Ner & 20 & 4979 \\
\hline $\begin{array}{l}\text { Neocalyptrella robusta (Norman) } \\
\text { Hernández-Becerril et Meave }\end{array}$ & $\mathrm{R}, \mathrm{B}$ & 9.1 & $\mathrm{~F}$ & $\mathrm{P}$ & 2 & Tr, Ner, Oc & 20 & 557 \\
\hline Nitzschia bicapitata Cleve & $\mathrm{R}$ & 9.4 & $\mathrm{~F}$ & $\mathrm{P}$ & 2 & $\mathrm{Tr}, \mathrm{Tm}, \mathrm{Oc}$ & 40 & 4025 \\
\hline N. bifurcata Kaczmarska et Licea & $\mathrm{B}$ & 4.2 & $\mathrm{~F}$ & $\mathrm{P}$ & 2 & $\mathrm{Tr}, \mathrm{Tm}, \mathrm{Oc}$ & 40 & 1405 \\
\hline N. gandersheimiensis Krasske & $\mathrm{B}$ & 3.3 & $\mathrm{~F}$ & $P$ & 2 & $\mathrm{Tr}, \mathrm{Sbt}, \mathrm{Slb}$ & 320 & 5556 \\
\hline N. longissima (Brébisson) Grunow & $\mathrm{R}, \mathrm{B}$ & 19.7 & $\mathrm{~F}$ & $\mathrm{~T}$ & 2 & Ner, Oc & 10 & 2506 \\
\hline N. pacifica Cupp & $\mathrm{R}$ & 0.3 & $\mathrm{~F}$ & $\mathrm{P}$ & 2 & $\mathrm{Tr}, \mathrm{Tm}, \mathrm{Oc}$ & & \\
\hline N. sicula (Castracane) Hustedt & $\mathrm{R}, \mathrm{B}$ & 5.5 & $\mathrm{~F}$ & $\mathrm{~T}$ & 2 & Tr, Ner & 20 & 3809 \\
\hline N. sigma (Kützing) W. Smith & $\mathrm{R}, \mathrm{B}$ & 3.64 & $\mathrm{~F}$ & $\mathrm{~T}$ & 2 & $\mathrm{Tr}, \mathrm{Sbt}, \mathrm{Slb}$ & 10 & 2506 \\
\hline N. sigmoidea (Nitzsch) W. Smith & $\mathrm{B}$ & 2.4 & $\mathrm{~F}$ & $\mathrm{~T}$ & 2 & Tr, Ner & 20 & 5882 \\
\hline N. socialis Gregory & $\mathrm{R}$ & 0.3 & $\mathrm{~F}$ & $\mathrm{~T}$ & 2 & $\mathrm{Tr}, \mathrm{Ner}, \mathrm{Oc}$ & & \\
\hline$N . \mathrm{sp}$. & $\mathrm{B}$ & 36.9 & $\mathrm{~F}$ & $P$ & 2 & Tr, Ner & 49 & 61172 \\
\hline Odontella aurita (Lyngbye) Agardh & $\mathrm{R}, \mathrm{B}$ & 20.0 & $\mathrm{~F}$ & $\mathrm{~T}$ & 2 & $\mathrm{Tr}, \mathrm{Tm}, \mathrm{Ner}, \mathrm{Oc}$ & 20 & 7388 \\
\hline O. longicruris (Greville) Hoban & $\mathrm{R}, \mathrm{B}$ & 16.1 & $\mathrm{~F}$ & $\mathrm{~T}$ & 4 & $\mathrm{Tr}, \mathrm{Tm}, \mathrm{Ner}$ & 13 & 13335 \\
\hline O. mobiliensis (Bailey) Grunow & $\mathrm{R}, \mathrm{B}$ & 10.9 & $\mathrm{~F}$ & $P$ & 2 & Tr, Ner, Oc & 20 & 1162 \\
\hline O. sinensis (Greville) Grunow & $\mathrm{R}, \mathrm{B}$ & 0.9 & $\mathrm{~F}$ & $\mathrm{P}$ & 4 & Tr, Ner, Oc & 48 & 1940 \\
\hline Paralia sulcata (Ehrenberg) Cleve & $\mathrm{R}, \mathrm{B}$ & 3.3 & $\mathrm{~F}$ & $\mathrm{~T}$ & 4 & Tr, Tm, Ner, Oc & 120 & 16035 \\
\hline Petroneis sp. & $\mathrm{B}$ & 0.3 & $\mathrm{~F}$ & $\mathrm{~T}$ & 2 & $\operatorname{Tr}, \mathrm{Tm}, \mathrm{Ner}$ & & 380 \\
\hline Pinnularia sp. & $\mathrm{B}$ & 1.2 & $\mathrm{~F}$ & $\mathrm{~T}$ & 2 & Tr, Ner & 27 & 163 \\
\hline Plagiogramma tessellatum Greville & $\mathrm{R}, \mathrm{B}$ & 0.6 & $\mathrm{~F}$ & $\mathrm{~T}$ & 2 & Tr, Sbt, Ner & 56 & 178 \\
\hline $\begin{array}{l}\text { Plagiotropis lepidoptera (Gregory) } \\
\text { Kuntze }\end{array}$ & $\mathrm{R}, \mathrm{B}$ & 2.4 & $\mathrm{~F}$ & $\mathrm{P}, \mathrm{T}$ & 2 & $\mathrm{Tr}, \mathrm{Ner}, \mathrm{Oc}$ & 20 & 2965 \\
\hline $\begin{array}{l}\text { Planktoniella muriformis (Loeblich, } \\
\text { Wight et Darley) Round }\end{array}$ & $\mathrm{R}, \mathrm{B}$ & 4.2 & $\mathrm{~F}$ & $\mathrm{P}, \mathrm{T}$ & 3 & $\mathrm{Tr}, \mathrm{Tm}, \mathrm{Ner}, \mathrm{Oc}$ & 42 & 208 \\
\hline P. sol (Wallich) Schütt & $\mathrm{R}, \mathrm{B}$ & 27.9 & $\mathrm{~F}$ & $\mathrm{P}$ & 2 & Tr, Tm, Ner, Oc & 10 & 824 \\
\hline $\begin{array}{l}\text { Pleurosigma angulatum (Queckett) } \\
\text { W. Smith }\end{array}$ & $\mathrm{R}, \mathrm{B}$ & 7.3 & $\mathrm{~F}$ & $\mathrm{P}, \mathrm{T}$ & 2 & Tr, Ner & 178 & 215 \\
\hline P. diverse-striatum Meister & $\mathrm{R}, \mathrm{B}$ & 10.0 & $\mathrm{~F}$ & $\mathrm{P}$ & 2 & $\mathrm{Tr}, \mathrm{Tm}, \mathrm{Ner}$ & 13 & 2138 \\
\hline P. formosum W. Smith & $\mathrm{R}$ & 3.0 & $\mathrm{~F}$ & $\mathrm{P}, \mathrm{T}$ & 2 & Sbt, Ner & & \\
\hline P. normanii Ralfs & $\mathrm{R}, \mathrm{B}$ & 2.1 & $\mathrm{~F}$ & $\mathrm{P}$ & 2 & Ner, Oc & 45 & 65 \\
\hline P. rhombeum Grunow & $\mathrm{R}, \mathrm{B}$ & 7.3 & $\mathrm{~F}$ & $\mathrm{P}$ & 2 & Tr, Ner & 19 & 246 \\
\hline P. sp. 1 & $\mathrm{R}$ & 1.2 & $\mathrm{~F}$ & $\mathrm{P}$ & 2 & Tr, Ner & & \\
\hline P. sp. 2 & $\mathrm{R}$ & 0.3 & $\mathrm{~F}$ & $\mathrm{P}$ & 2 & Tr, Ner & & \\
\hline Podocystis adriatica (Kützing) Ralfs & $\mathrm{R}$ & 0.3 & $\mathrm{~F}$ & $\mathrm{~T}$ & 2 & Ner & & \\
\hline
\end{tabular}


Apéndice 1. Continuación.

\begin{tabular}{|c|c|c|c|c|c|c|c|c|}
\hline \multirow[b]{2}{*}{ Taxa } & \multirow[b]{2}{*}{$\mathrm{M}$} & \multirow[b]{2}{*}{ Frec. } & \multirow[b]{2}{*}{$\mathrm{N}$} & \multirow[b]{2}{*}{$\mathrm{H}$} & \multirow[b]{2}{*}{$\mathrm{NO}$} & \multirow{2}{*}{$\begin{array}{l}\text { Distribución } \\
\text { Global }\end{array}$} & \multicolumn{2}{|c|}{$\begin{array}{l}\text { Abundancia } \\
\text { céls. } \mathrm{L}^{-1}\end{array}$} \\
\hline & & & & & & & Mín. & Máx. \\
\hline $\begin{array}{l}\text { Proboscia alata (Brightwell) } \\
\text { Sundström }\end{array}$ & $\mathrm{R}, \mathrm{B}$ & 56.4 & $\mathrm{~F}$ & $\mathrm{P}$ & 4 & Sbt, Tm, Ner, Oc & 10 & 7388 \\
\hline $\begin{array}{l}\text { P. alata f. gracillima (Cleve) Licea et } \\
\text { Moreno }\end{array}$ & $\mathrm{R}, \mathrm{B}$ & 8.2 & $\mathrm{~F}$ & $\mathrm{P}$ & 2 & Sbt, Tm, Ner & 86 & 7989 \\
\hline $\begin{array}{l}\text { P. indica (H. Peragallo) Hernández- } \\
\text { Becerril }\end{array}$ & $\mathrm{R}, \mathrm{B}$ & 10.3 & $\mathrm{~F}$ & $\mathrm{P}$ & 2 & $\mathrm{Tr}, \mathrm{Sbt}$ & 48 & 15497 \\
\hline $\begin{array}{l}\text { P. cf. eumorpha Takahashi, Jordan et } \\
\text { Priddle }\end{array}$ & B & 0.3 & $\mathrm{~F}$ & $\mathrm{P}$ & 2 & $\mathrm{Tr}, \mathrm{Oc}$ & & 150 \\
\hline $\begin{array}{l}\text { Psammodictyon constrictum } \\
\text { (Gregory) D.G. Mann }\end{array}$ & B & 1.5 & $\mathrm{~F}$ & $\mathrm{P}$ & 2 & $\mathrm{Tr}, \mathrm{Sbt}, \mathrm{Oc}$ & 46 & 456 \\
\hline P. panduriforme (Gregory) D.G. Mann & B & 0.3 & $\mathrm{~F}$ & PT & 2 & Tr, Ner, Oc & & 1265 \\
\hline Pseudoguinardia recta von Stosch & $\mathrm{R}, \mathrm{B}$ & 31.5 & $\mathrm{~F}$ & $\mathrm{P}$ & 4 & Tr, Ner, Oc & 40 & 9145 \\
\hline $\begin{array}{l}\text { Pseudo-nitzschia delicatissima } \\
\text { (Cleve) Heiden** }\end{array}$ & $\mathrm{R}, \mathrm{B}$ & 12.1 & $\mathrm{~F}$ & $P$ & 4 & Tm, Tr, Ner, Oc & 258 & 133909 \\
\hline $\begin{array}{l}\text { P. pseudodelicatissima (Hasle) } \\
\text { Hasle** }\end{array}$ & $\mathrm{R}, \mathrm{B}$ & 51.5 & $\mathrm{~F}$ & $P$ & 4 & Ner, Oc & 283 & 306411 \\
\hline $\begin{array}{l}\text { P. pungens (Grunow ex Cleve) Hasle } \\
\text { var. pungens* }\end{array}$ & $\mathrm{R}, \mathrm{B}$ & 48.2 & $\mathrm{~F}$ & $\mathrm{P}$ & 4 & Ner, Oc & 55 & 160866 \\
\hline $\begin{array}{l}\text { P. pungens var. atlantica (Grunow et } \\
\text { Cleve) Hasle }\end{array}$ & $\mathrm{R}, \mathrm{B}$ & 8.8 & $\mathrm{~F}$ & $\mathrm{P}$ & 4 & Ner & 653 & 10452 \\
\hline P. cf. seriata (Cleve) H. Peragallo** & $\mathrm{R}, \mathrm{B}$ & 7.88 & $\mathrm{~F}$ & $\mathrm{P}$ & 4 & Ner, Oc & 327 & 56904 \\
\hline P. subfraudulenta (Hasle) Hasle & $\mathrm{R}, \mathrm{B}$ & 48.2 & $\mathrm{~F}$ & $\mathrm{P}$ & 4 & Tr, Tm, Ner & 36 & 55831 \\
\hline P. sp. & B & 41.9 & $\mathrm{~F}$ & $\mathrm{P}$ & 4 & Tr, Ner & 89 & 15436 \\
\hline $\begin{array}{l}\text { Pseudosolenia calcar-avis (Schultze) } \\
\text { Sundström }\end{array}$ & $\mathrm{R}, \mathrm{B}$ & 20.3 & $\mathrm{~F}$ & $\mathrm{P}$ & 2 & Sbt, Tm, Ner, Oc & 16 & 5031 \\
\hline $\begin{array}{l}\text { Rhizosolenia acuminata }(\mathrm{H} . \\
\text { Peragallo) Cleve }\end{array}$ & $\mathrm{R}, \mathrm{B}$ & 10.3 & $\mathrm{~F}$ & $\mathrm{P}$ & 2 & $\mathrm{Tr}$, Ner, Oc & 10 & 89 \\
\hline R. bergonii H. Peragallo & $\mathrm{R}, \mathrm{B}$ & 13.3 & $\mathrm{~F}$ & $\mathrm{P}$ & 2 & $\mathrm{Tr}, \mathrm{Oc}$ & 20 & 511 \\
\hline R. castracanei H. Peragallo & $\mathrm{R}$ & 1.8 & $\mathrm{~F}$ & $P$ & 2 & $\mathrm{Tr}, \mathrm{Oc}$ & & \\
\hline R. clevei Ostenfeld var. clevei & $\mathrm{R}, \mathrm{B}$ & 5.8 & $\mathrm{~F}$ & $\mathrm{P}$ & 2 & $\mathrm{Tr}, \mathrm{Oc}$ & 43 & 87 \\
\hline R. clevei var. communis Sundström & $\mathrm{R}, \mathrm{B}$ & 1.8 & $\mathrm{~F}$ & $\mathrm{P}$ & 2 & Sbt, Tm, Ner & 18 & 119 \\
\hline R. crassa Schimper & $\mathrm{R}$ & 0.3 & $\mathrm{~F}$ & $P$ & 2 & Fr, HS, Oc & 20 & 8493 \\
\hline R. hebetata J.W. Bailey & $\mathrm{R}, \mathrm{B}$ & 12.1 & $\mathrm{~F}$ & $\mathrm{P}$ & 2 & $\begin{array}{c}\text { Tm, HN, Ner, } \\
\text { Oc }\end{array}$ & 20 & 2691 \\
\hline R. hyalina Ostenfeld & $\mathrm{R}, \mathrm{B}$ & 10.0 & $\mathrm{~F}$ & $\mathrm{P}$ & 4 & Tr, Ner, Oc & 48 & 11112 \\
\hline R. imbricata Brightwell & $\mathrm{R}, \mathrm{B}$ & 38.2 & $\mathrm{~F}$ & $\mathrm{P}$ & 4 & Sbt, Tm, Ner, Oc & 22 & 17562 \\
\hline R. pungens Cleve-Euler & $\mathrm{R}, \mathrm{B}$ & 62.5 & $\mathrm{~F}$ & $\mathrm{P}$ & 2 & $\mathrm{Tr}, \mathrm{Sbt}, \mathrm{Oc}$ & 10 & 20790 \\
\hline R. setigera Brightwell & $\mathrm{R}, \mathrm{B}$ & 18.2 & $\mathrm{~F}$ & $\mathrm{P}$ & 4 & Sbt, Tm, Ner, Oc & 40 & 14717 \\
\hline
\end{tabular}


Apéndice 1. Continuación.

\begin{tabular}{|c|c|c|c|c|c|c|c|c|}
\hline \multirow[b]{2}{*}{ Таха } & \multirow[b]{2}{*}{$\mathrm{M}$} & \multirow[b]{2}{*}{ Frec. } & \multirow[b]{2}{*}{$\mathrm{N}$} & \multirow[b]{2}{*}{$\mathrm{H}$} & \multirow[b]{2}{*}{ NO } & \multirow{2}{*}{$\begin{array}{l}\text { Distribución } \\
\text { Global }\end{array}$} & \multicolumn{2}{|c|}{$\begin{array}{l}\text { Abundancia } \\
\text { céls. } \mathrm{L}^{-1}\end{array}$} \\
\hline & & & & & & & Mín. & Máx. \\
\hline R. styliformis Brightwell & $\mathrm{R}, \mathrm{B}$ & 24.2 & $\mathrm{~F}$ & $\mathrm{P}$ & 2 & $\begin{array}{l}\text { Tm, HN, Ner, } \\
\text { Oc }\end{array}$ & 160 & 28286 \\
\hline R. temperei H. Peragallo & $\mathrm{R}, \mathrm{B}$ & 5.5 & $\mathrm{~F}$ & $\mathrm{P}$ & 2 & $\mathrm{Tm}, \mathrm{Oc}$ & 41 & 2277 \\
\hline $\begin{array}{l}\text { Rhopalodia gibberula (Ehrenberg) } \\
\text { Müller }\end{array}$ & $\mathrm{R}, \mathrm{B}$ & 3.0 & $\mathrm{~F}$ & $\mathrm{~T}$ & 2 & Ner, eurihalina & 35 & 3271 \\
\hline $\begin{array}{l}\text { Roperia tesselata (Roper) Grunow in } \\
\text { van Heurck }\end{array}$ & B & 2.4 & $\mathrm{~F}$ & $\mathrm{P}$ & 2 & $\mathrm{Tr}, \mathrm{Tm}, \mathrm{Ner}$ & 22 & 2453 \\
\hline $\begin{array}{l}\text { Skeletonema cf. pseudocostatum } \\
\text { Medlin }\end{array}$ & $\mathrm{R}, \mathrm{B}$ & 36.7 & $\mathrm{~F}$ & $\mathrm{P}$ & 4 & Tm, Tr, Ner & 20 & 85501 \\
\hline S. tropicum Cleve & $\mathrm{R}, \mathrm{B}$ & 13.3 & $\mathrm{~F}$ & $\mathrm{P}$ & 4 & Tr, Ner & 48 & 142261 \\
\hline S. sp. 1 & $\mathrm{R}, \mathrm{B}$ & 29.4 & $\mathrm{~F}$ & $\mathrm{P}$ & 4 & Tr, Ner & 10 & 268110 \\
\hline S. sp. 2 & $\mathrm{~B}$ & 0.3 & $\mathrm{~F}$ & $\mathrm{P}$ & 4 & Tr, Ner & 10 & 6686 \\
\hline Spatangidium arachne Brébisson & $\mathrm{R}, \mathrm{B}$ & 1.2 & $\mathrm{~F}$ & $\mathrm{P}$ & 2 & Tr, Ner & 32 & 1069 \\
\hline $\begin{array}{l}\text { Stellarima stellaris (Roper) Hasle et } \\
\text { Sims }\end{array}$ & B & 7.9 & $\mathrm{~F}$ & $\mathrm{P}$ & 2 & $\mathrm{Tr}, \mathrm{Tm}, \mathrm{Oc}$ & 43 & 2323 \\
\hline $\begin{array}{l}\text { Stephanopyxis palmeriana (Greville) } \\
\text { Grunow }\end{array}$ & $\mathrm{R}$ & 1.2 & $\mathrm{~F}$ & $\mathrm{P}$ & 4 & $\mathrm{Tm}, \mathrm{Tr}, \mathrm{Oc}$ & & \\
\hline S. turris (Greville et Arnott) Ralfs & B & 12.7 & $\mathrm{~F}$ & $\mathrm{P}$ & 4 & $\operatorname{Tm}, \operatorname{Tr}$ & 56 & 2347 \\
\hline $\begin{array}{l}\text { Surirella fastuosa Ehrenberg var. } \\
\text { fastuosa }\end{array}$ & $\mathrm{R}, \mathrm{B}$ & 12.4 & $\mathrm{~F}$ & $\mathrm{~T}$ & 2 & Tr, Ner & 20 & 549 \\
\hline $\begin{array}{l}\text { S. fastuosa var. recedens (A. } \\
\text { Schmidt) Cleve }\end{array}$ & $\mathrm{R}, \mathrm{B}$ & 5.8 & $\mathrm{~F}$ & $\mathrm{~T}$ & 2 & $\mathrm{Tr}, \mathrm{Sbt}$ & 24 & 139 \\
\hline S. febigerii Lewis & $\mathrm{R}$ & 1.5 & $\mathrm{~F}$ & $\mathrm{P}$ & 2 & Sbt, Ner & & \\
\hline Synedra ulna (Nitzsch) Ehrenberg & $\mathrm{R}, \mathrm{B}$ & 2.4 & $\mathrm{~F}$ & $\mathrm{~T}$ & 2 & Sbt, Tm, Ner & 27 & 453 \\
\hline S. sp. 1 & B & 0.6 & $\mathrm{~F}$ & $\mathrm{P}$ & 2 & Tr, Ner & 29 & 352 \\
\hline $\begin{array}{l}\text { Thalassionema bacillaris (Heiden) } \\
\text { Kolbe }\end{array}$ & $\mathrm{R}, \mathrm{B}$ & 15.5 & $\mathrm{~F}$ & $\mathrm{P}$ & 2 & Tr, Ner & 127 & 8791 \\
\hline $\begin{array}{l}\text { T. frauenfeldii (Grunow) Tempère et } \\
\text { H. Peragallo ex Hallegraeff }\end{array}$ & $\mathrm{R}, \mathrm{B}$ & 51.5 & $\mathrm{~F}$ & $\mathrm{P}$ & 4 & $\mathrm{Tr}, \mathrm{Tm}, \mathrm{Ner}, \mathrm{Oc}$ & 42 & 35390 \\
\hline $\begin{array}{l}\text { T. nitzschioides (Grunow) Tempère } \\
\text { et H. Peragallo ex Hallegraeff var. } \\
\text { nitzschioides }\end{array}$ & $\mathrm{R}, \mathrm{B}$ & 67.6 & $\mathrm{~F}$ & $\mathrm{P}$ & 4 & Tm, Tr, Ner & 10 & 66417 \\
\hline $\begin{array}{l}\text { T. nitzschioides var. capitulata } \\
\text { (Castracane) Moreno }\end{array}$ & $\mathrm{R}$ & 0.6 & $\mathrm{~F}$ & $\mathrm{P}$ & 4 & Tm, Ner & & \\
\hline $\begin{array}{l}\text { T. nitzschioides var. claviformis } \\
\text { (Schrader) Moreno }\end{array}$ & $\mathrm{R}$ & 0.3 & $\mathrm{~F}$ & $\mathrm{P}$ & 4 & $\mathrm{Tr}, \mathrm{Tm}, \mathrm{Slb}, ?$ & & \\
\hline $\begin{array}{l}\text { T. nitzschioides var. parva Heiden et } \\
\text { Kolbe }\end{array}$ & $\mathrm{R}, \mathrm{B}$ & 1.2 & $\mathrm{~F}$ & $\mathrm{P}$ & 4 & $\mathrm{Tr}, \mathrm{Sbt}$ & 100 & 5216 \\
\hline
\end{tabular}


Apéndice 1. Continuación.

\begin{tabular}{|c|c|c|c|c|c|c|c|c|}
\hline \multirow[b]{2}{*}{ Taxa } & \multirow[b]{2}{*}{$\mathrm{M}$} & \multirow[b]{2}{*}{ Frec. } & \multirow[b]{2}{*}{$\mathrm{N}$} & \multirow[b]{2}{*}{$\mathrm{H}$} & \multirow[b]{2}{*}{ NO } & \multirow{2}{*}{$\begin{array}{l}\text { Distribución } \\
\text { Global }\end{array}$} & \multicolumn{2}{|c|}{$\begin{array}{c}\text { Abundancia } \\
\text { céls. } \mathrm{L}^{-1}\end{array}$} \\
\hline & & & & & & & Mín. & Máx. \\
\hline $\begin{array}{l}\text { Thalassiosira decipiens (Grunow) E. } \\
\text { Jörgensen }\end{array}$ & $\mathrm{B}$ & 2.1 & $\mathrm{~F}$ & $\mathrm{P}$ & 2 & Sbt, Tm, Ner, Oc & 29 & 1704 \\
\hline T. eccentrica (Ehrenberg) Cleve & $\mathrm{R}, \mathrm{B}$ & 3.9 & $\mathrm{~F}$ & $\mathrm{P}$ & 2 & Tr, Tm, Ner, Oc & 32 & 1417 \\
\hline T. hendeyi Hasle et Fryxell & $\mathrm{R}$ & 1.5 & $\mathrm{~F}$ & $\mathrm{P}$ & 2 & $\mathrm{Tr}, \mathrm{Oc}$ & & \\
\hline T. leptopus (Grunow) Hasle et Fryxell & $\mathrm{R}, \mathrm{B}$ & 6.0 & $\mathrm{~F}$ & $\mathrm{~T}$ & 2 & $\mathrm{Tr}, \mathrm{Sbt}, \mathrm{Ner}$ & 54 & 2347 \\
\hline T. minuscula Krasske* & $\mathrm{B}$ & 4.8 & $\mathrm{~F}$ & $\mathrm{P}$ & 2 & Sbt, Tm, Ner, Oc & 43 & 14250 \\
\hline T. oestrupii (Ostenfeld) Hasle & $\mathrm{R}$ & 0.3 & $\mathrm{~F}$ & $\mathrm{~T}$ & 2 & Tr, Tm, Ner & & \\
\hline T. rotula Meunier* & $\mathrm{B}$ & 10.9 & $\mathrm{~F}$ & $\mathrm{P}$ & 4 & Sbt, Tm, Ner & 27 & 8453 \\
\hline T. simonsenii Hasle et Fryxell & $\mathrm{R}$ & 1.5 & $\mathrm{~F}$ & $\mathrm{~T}$ & 2 & Sbt, Ner & & \\
\hline T. subtilis (Ostenfeld) Gran* & B & 2.4 & $\mathrm{~F}$ & $\mathrm{P}$ & 2 & Tr, Tm, Ner, Oc & 31 & 1041 \\
\hline T. sp. 1 & B & 4.8 & $\mathrm{~F}$ & $\mathrm{P}$ & 2 & Tr, Ner & 27 & 8453 \\
\hline T. sp. 2 & $\mathrm{R}, \mathrm{B}$ & 0.3 & $\mathrm{~F}$ & $\mathrm{P}$ & 2 & Tr, Ner & 43 & 856 \\
\hline $\begin{array}{l}\text { Thalassiothrix longissima Cleve et } \\
\text { Grunow }\end{array}$ & $\mathrm{R}, \mathrm{B}$ & 11.5 & $\mathrm{~F}$ & $\mathrm{P}$ & 2 & $\begin{array}{l}\text { Fr, Tm, HN, } \\
\text { Ner, Oc }\end{array}$ & 38 & 4497 \\
\hline Trachyneis aspera (Ehrenberg) Cleve & B & 0.3 & $\mathrm{~F}$ & $\mathrm{~T}$ & 2 & Sbt, Tm, Ner & & 125 \\
\hline Triceratium dubium Brightwell & $\mathrm{R}, \mathrm{B}$ & 0.9 & $\mathrm{~F}$ & $\mathrm{~T}$ & 2 & Tr, Sbt, Ner & 150 & 2147 \\
\hline T. favus Ehrenberg & $\mathrm{R}, \mathrm{B}$ & 2.4 & $\mathrm{~F}$ & $\mathrm{P}$ & 2 & Cos, Ner, Oc & 20 & 250 \\
\hline Tryblionella punctata W. Smith & B & 0.6 & $\mathrm{~F}$ & $\mathrm{~T}$ & 2 & $\operatorname{Tr}, \operatorname{Tm}$ & 22 & 352 \\
\hline \multicolumn{9}{|l|}{ División Dinophyta } \\
\hline Achradina pulchra Lohmann & $\mathrm{B}, \mathrm{V}$ & 12.4 & $\mathrm{H}$ & $\mathrm{P}$ & 1 & Tr, Ner, Oc & 20 & 7718 \\
\hline $\begin{array}{l}\text { Akashiwo sanguinea (Hirasaka) G. } \\
\text { Hansen et O. Moestrup** }\end{array}$ & $\mathrm{R}, \mathrm{B}, \mathrm{V}$ & 78.8 & $\mathrm{~F}$ & $\mathrm{P}$ & 1 & Tm, Tr, Ner & 10 & 19844 \\
\hline $\begin{array}{l}\text { Alexandrium catenella (Whedon et } \\
\text { Kofoid) Balech** }\end{array}$ & $\mathrm{R}$ & 0.3 & $\mathrm{~F}$ & $\mathrm{P}$ & 4 & Fr & & \\
\hline A. fraterculus (Balech) Balech & $\mathrm{B}$ & 3.3 & $\mathrm{~F}$ & $\mathrm{P}$ & 4 & Tr, Ner, Oc & 20 & 595 \\
\hline $\begin{array}{l}\text { A. cf. ostenfeldii (Paulsen) Balech et } \\
\text { Tangen** }\end{array}$ & B & 3.0 & $\mathrm{~F}$ & $\mathrm{P}$ & 4 & $\mathrm{Tm}, \mathrm{Fr}$ & 20 & 3066 \\
\hline A. cf. tamarense (Lebour) Balech & $\mathrm{B}$ & 19.1 & $\mathrm{~F}$ & $\mathrm{P}$ & 4 & Ner & 19 & 2613 \\
\hline A. sp. & $\mathrm{R}$ & 4.2 & $\mathrm{~F}$ & $\mathrm{P}$ & 1 & Tr, Ner & 10 & 3319 \\
\hline Amphidinium acutissimum Schiller & B & 11.2 & $\mathrm{~F}$ & $\mathrm{P}$ & 1 & $\mathrm{Tm}, \mathrm{Oc}$ & 20 & 2134 \\
\hline A. extensum Wulff & B & 0.6 & $\mathrm{H}$ & $\mathrm{P}$ & 1 & $\mathrm{Tr}, \mathrm{Tm}, \mathrm{Ner}$ & 30 & 60 \\
\hline $\begin{array}{l}\text { A. gibbosum (Maranda et Shimizu) } \\
\text { Jørgensen et Murray }\end{array}$ & $\mathrm{B}, \mathrm{V}$ & 0.6 & $\mathrm{~F}$ & $\mathrm{P}$ & 1 & Tm, Tr, Ner & 40 & 178 \\
\hline A. massartiae Biecheler & B & 0.3 & $\mathrm{~F}$ & $\mathrm{P}$ & 1 & Tm, Ner & 20 & 1778 \\
\hline Amphisolenia bidentata Schröder & $\mathrm{R}, \mathrm{B}$ & 8.5 & $\mathrm{~F}$ & $\mathrm{P}$ & 1 & $\mathrm{Tm}, \mathrm{Tr}, \mathrm{Oc}$ & 29 & 653 \\
\hline A. cf. truncata Kofoid et Michener & $\mathrm{R}$ & 0.6 & $\mathrm{~F}$ & $\mathrm{P}$ & 1 & $\mathrm{Tr}, \mathrm{Oc}$ & & \\
\hline Asterodinium gracile Sournia & $\mathrm{B}$ & 1.5 & $\mathrm{~F}$ & $\mathrm{P}$ & 1 & $\mathrm{Tr}, \mathrm{Tm}, \mathrm{Oc}$ & 20 & 653 \\
\hline
\end{tabular}


Apéndice 1. Continuación.

\begin{tabular}{|c|c|c|c|c|c|c|c|c|}
\hline \multirow[b]{2}{*}{ Taxa } & \multirow[b]{2}{*}{$\mathrm{M}$} & \multirow[b]{2}{*}{ Frec. } & \multirow[b]{2}{*}{$\mathrm{N}$} & \multirow[b]{2}{*}{$\mathrm{H}$} & \multirow[b]{2}{*}{ NO } & \multirow{2}{*}{$\begin{array}{l}\text { Distribución } \\
\text { Global }\end{array}$} & \multicolumn{2}{|c|}{$\begin{array}{c}\text { Abundancia } \\
\text { céls. } \mathrm{L}^{-1}\end{array}$} \\
\hline & & & & & & & Mín. & Máx. \\
\hline $\begin{array}{l}\text { Balechina coerulea (Dogiel) F.J.R. } \\
\text { Taylor }\end{array}$ & $\mathrm{R}, \mathrm{B}$ & 12.1 & $\mathrm{H}$ & $\mathrm{P}$ & 1 & Tr, Tm, Ner, Oc & 20 & 3809 \\
\hline $\begin{array}{l}\text { Blepharocysta splendormaris } \\
\text { (Ehrenberg) Ehrenberg }\end{array}$ & $\mathrm{R}, \mathrm{B}$ & 10.6 & $\mathrm{H}$ & $\mathrm{P}$ & 1 & Tr, Tm, Ner, Oc & 14 & 721 \\
\hline Brachydinum capitatum F.J.R. Taylor & B & 1.8 & $\mathrm{~F}$ & $\mathrm{P}$ & 1 & Tm, Tr, Ner, Oc & 20 & 327 \\
\hline Ceratocorys armata (Schütt) Kofoid & $\mathrm{R}$ & 1.2 & $\mathrm{~F}$ & $\mathrm{P}$ & 1 & $\mathrm{Tm}, \mathrm{Tr}, \mathrm{Ner}, \mathrm{Oc}$ & & \\
\hline C. bipes (Cleve) Kofoid & $\mathrm{R}$ & 0.6 & $\mathrm{~F}$ & $\mathrm{P}$ & 1 & Tr, Ner, Oc & & \\
\hline C. horrida Stein & $\mathrm{R}, \mathrm{B}$ & 15.2 & $\mathrm{~F}$ & $\mathrm{P}$ & 1 & $\mathrm{Tr}, \mathrm{Tm}, \mathrm{Ner}, \mathrm{Oc}$ & 20 & 653 \\
\hline C. reticulata Graham & $\mathrm{R}$ & 0.6 & $\mathrm{~F}$ & $\mathrm{P}$ & 1 & Tr, Ner, Oc & & \\
\hline Ceratoperidinium yeye Margalef & B & 0.6 & $\mathrm{~F}$ & $\mathrm{P}$ & 1 & $\mathrm{Tm}, \mathrm{Oc}$ & 38 & 45 \\
\hline $\begin{array}{l}\text { Chytriodinium affine (Dogiel) } \\
\text { Chatton }\end{array}$ & B & 10.6 & $\mathrm{H}$ & $\mathrm{P}$ & 1 & $\mathrm{Tm}, \mathrm{Tr}, \mathrm{Ner}$ & 18 & 3512 \\
\hline $\begin{array}{l}\text { Cochlodinium convolutum Kofoid et } \\
\text { Swezy }\end{array}$ & $\mathrm{V}$ & 0.9 & $\mathrm{~F}$ & $\mathrm{P}$ & 4 & Tr, Tm, Ner & & \\
\hline $\begin{array}{l}\text { C. cf. archimedes (Pouchet) } \\
\text { Lemmermann }\end{array}$ & B & 0.3 & $\mathrm{H}$ & $\mathrm{P}$ & 1 & Tm, Ner, Oc & 20 & 153 \\
\hline $\begin{array}{l}\text { C. fulvecens Iwataki, Kawami et } \\
\text { Matsuoka }\end{array}$ & V & 0.3 & $\mathrm{~F}$ & $\mathrm{P}$ & 4 & Tr, Tm, Ner & & \\
\hline C. polykrikoides Margalef** & B & 0.9 & $\mathrm{~F}$ & $\mathrm{P}$ & 4 & $\mathrm{Tm}, \mathrm{Tr}, \mathrm{Ner}$ & 10 & 1778 \\
\hline $\begin{array}{l}\text { Corythodinium belgicae (Maunier) } \\
\text { Taylor }\end{array}$ & B & 0.9 & $\mathrm{~F}$ & $\mathrm{P}$ & 1 & $\mathrm{Tm}, \mathrm{Tr}, \mathrm{Ner}, \mathrm{Oc}$ & 20 & 40 \\
\hline C. constrictum (Stein) Taylor & B & 0.6 & $\mathrm{~F}$ & $\mathrm{P}$ & 1 & $\mathrm{Tr}, \mathrm{Tm}, \mathrm{Ner}, \mathrm{Oc}$ & 35 & 43 \\
\hline C. diploconus (Stein) Taylor & B & 0.3 & $\mathrm{~F}$ & $\mathrm{P}$ & 1 & $\mathrm{Tm}, \mathrm{Tr}, \mathrm{Oc}$ & & 4195 \\
\hline C. elegans (Pavillard) Taylor & B & 0.3 & $\mathrm{~F}$ & $\mathrm{P}$ & 1 & $\mathrm{Tr}, \mathrm{Tm}, \mathrm{Oc}$ & & 1417 \\
\hline C. frenguellii (Rampi) Taylor & B & 0.6 & $\mathrm{~F}$ & $\mathrm{P}$ & 1 & $\mathrm{Tr}, \mathrm{Tm}, \mathrm{Oc}$ & 29 & 38 \\
\hline $\begin{array}{l}\text { C. tesselatum (Stein) Loeblich Jr. et } \\
\text { Loeblich III }\end{array}$ & B & 0.3 & $\mathrm{~F}$ & $\mathrm{P}$ & 1 & $\mathrm{Tm}, \mathrm{Tr}, \mathrm{Oc}$ & & 106 \\
\hline $\begin{array}{l}\text { Dinophysis acuminata Claparède et J. } \\
\text { Lachmann** }\end{array}$ & B & 1.5 & M & $\mathrm{P}$ & 1 & $\operatorname{Tr}, \mathrm{Tm}, \mathrm{Ner}, \mathrm{Oc}$ & 20 & 171 \\
\hline D. bibulbus Balech & B & 1.2 & M & $\mathrm{P}$ & 1 & $\mathrm{Tr}, \mathrm{Tm}, \mathrm{Ner}, \mathrm{Oc}$ & 33 & 54 \\
\hline D. caudata Saville-Kent** & $\mathrm{R}, \mathrm{B}$ & 58.2 & M & $\mathrm{P}$ & 1 & Tr, Tm, Ner & 10 & 11112 \\
\hline D. cf. dens Pavillard & $\mathrm{B}$ & 0.3 & M & $\mathrm{P}$ & 1 & $\operatorname{Tr}, \operatorname{Tm}$ & & 2240 \\
\hline D. diegensis Kofoid & $\mathrm{R}, \mathrm{B}$ & 3.0 & M & $\mathrm{P}$ & 1 & $\mathrm{Tm}, \mathrm{Tr}, \mathrm{Ner}, \mathrm{Oc}$ & 20 & 1258 \\
\hline D. exigua Kofoid et Skogsberg & $\mathrm{R}, \mathrm{B}$ & 0.9 & M & $\mathrm{P}$ & 1 & $\mathrm{Tr}, \mathrm{Oc}$ & 15 & 20 \\
\hline D. fortii Pavillard** & $\mathrm{R}, \mathrm{B}$ & 8.2 & M & $\mathrm{P}$ & 1 & Tr, Sbt, Ner, Oc & 38 & 1601 \\
\hline D. hastata Stein & $\mathrm{R}$ & 0.6 & M & $\mathrm{P}$ & 1 & $\mathrm{Tm}, \mathrm{Tr}, \mathrm{Ner}, \mathrm{Oc}$ & & \\
\hline D. infundibulus Schiller & B & 0.3 & M & $\mathrm{P}$ & 1 & Tr, Ner & & 20 \\
\hline D. ovum Schütt & $\mathrm{R}$ & 0.3 & M & $\mathrm{P}$ & 1 & Sbt, HN, Oc & & \\
\hline
\end{tabular}


Apéndice 1. Continuación.

\begin{tabular}{|c|c|c|c|c|c|c|c|c|}
\hline \multirow[b]{2}{*}{ Taxa } & \multirow[b]{2}{*}{$\mathrm{M}$} & \multirow[b]{2}{*}{ Frec. } & \multirow[b]{2}{*}{$\mathrm{N}$} & \multirow[b]{2}{*}{$\mathrm{H}$} & \multirow[b]{2}{*}{$\mathrm{NO}$} & \multirow{2}{*}{$\begin{array}{l}\text { Distribución } \\
\text { Global }\end{array}$} & \multicolumn{2}{|c|}{$\begin{array}{c}\text { Abundancia } \\
\text { céls. } \mathrm{L}^{-1}\end{array}$} \\
\hline & & & & & & & Mín. & Máx. \\
\hline D. parvula (Schütt) Balech & $\mathrm{B}$ & 0.3 & M & $\mathrm{P}$ & 1 & Tm, Ner, Oc & & 20 \\
\hline D. pusilla Jörgensen & $\mathrm{B}$ & 0.6 & M & $\mathrm{P}$ & 1 & $\mathrm{Tm}, \mathrm{Oc}$ & 10 & 25 \\
\hline D. sacculus Stein** & $\mathrm{R}$ & 0.3 & M & $\mathrm{P}$ & 1 & Tm, Ner, Oc & & \\
\hline D. schuettii Murray et Whitting & $\mathrm{R}, \mathrm{B}$ & 3.6 & M & $\mathrm{P}$ & 1 & $\mathrm{Tm}, \mathrm{Tr}, \mathrm{Ner}, \mathrm{Oc}$ & 44 & 75 \\
\hline D. sp. 1 & B & 2.7 & M & $\mathrm{P}$ & 1 & Tr, Ner & 10 & 875 \\
\hline D. sp. 2 & B & 0.3 & M & $\mathrm{P}$ & 1 & Tr, Ner & & 10 \\
\hline Diplopsalis lenticula Bergh & $\mathrm{R}, \mathrm{B}$ & 41.8 & $\mathrm{H}$ & $\mathrm{P}$ & 1 & $\mathrm{Tm}, \mathrm{Tr}, \mathrm{Ner}, \mathrm{Oc}$ & 22 & 6984 \\
\hline $\begin{array}{l}\text { Diplopsalopsis bomba (Stein ex } \\
\text { Jörgensen) Dodge et Toriumi }\end{array}$ & $\mathrm{R}, \mathrm{B}$ & 66.4 & $\mathrm{H}$ & $\mathrm{P}$ & 1 & $\mathrm{Tm}, \mathrm{Tr}, \mathrm{Ner}, \mathrm{Oc}$ & 18 & 4252 \\
\hline D. globula Abé & $\mathrm{R}, \mathrm{B}$ & 1.2 & $\mathrm{H}$ & $\mathrm{P}$ & 1 & $\mathrm{Tm}, \mathrm{Tr}, \mathrm{Ner}, \mathrm{Oc}$ & 102 & 453 \\
\hline $\begin{array}{l}\text { Dissodinium pseudolunula Swift ex } \\
\text { Elbrächter et Drebes }\end{array}$ & $\mathrm{R}$ & 3.6 & $\mathrm{H}$ & $\mathrm{P}$ & 2 & Tm, Ner & & \\
\hline Ensiculifera angulata Balech & $\mathrm{R}, \mathrm{B}$ & 19.7 & $\mathrm{~F}$ & $\mathrm{~T}$ & 1 & Tr, Ner, Oc & 40 & 8813 \\
\hline E. mexicana Balech & $\mathrm{R}, \mathrm{B}$ & 1.5 & $\mathrm{~F}$ & $\mathrm{~T}$ & 1 & Tr, Tm, Ner, Oc & 1896 & 3977 \\
\hline $\begin{array}{l}\text { Erythropsidinium cf. agile (Hertwig) } \\
\text { P.C. Silva }\end{array}$ & B & 3.9 & $\mathrm{H}$ & $\mathrm{P}$ & 1 & $\mathrm{Tm}, \mathrm{Tr}, \mathrm{Oc}$ & 33 & 5032 \\
\hline Fragilidium mexicanum Balech & $\mathrm{R}, \mathrm{B}$ & 21.8 & M & $\mathrm{P}$ & 1 & Tr, Ner & 18 & 4984 \\
\hline$F$. sp. & B & 3.6 & M & $\mathrm{P}$ & 1 & Tr, Ner & 38 & 4025 \\
\hline $\begin{array}{l}\text { Goniodoma polyedricum (Pouchet) } \\
\text { Jörgensen }\end{array}$ & $\mathrm{R}, \mathrm{B}$ & 26.9 & $\mathrm{~F}$ & $\mathrm{P}$ & 1 & Tr, Tm, Ner & 10 & 3155 \\
\hline G. sphaericum Murray et Whitting & $\mathrm{R}, \mathrm{B}$ & 12.1 & $\mathrm{~F}$ & $\mathrm{P}$ & 1 & $\mathrm{Tr}, \mathrm{Sbt}, \mathrm{Oc}$ & 24 & 1140 \\
\hline Gonyaulax alaskensis Kofoid & $\mathrm{R}$ & 0.3 & $\mathrm{~F}$ & $\mathrm{P}$ & 1 & Tm, Ner, Oc & & \\
\hline G. birostris Stein & $\mathrm{R}, \mathrm{B}$ & 6.1 & $\mathrm{~F}$ & $\mathrm{P}$ & 1 & Tr, Ner, Oc & 10 & 257 \\
\hline G. diegensis Kofoid & $\mathrm{R}, \mathrm{B}$ & 6.9 & $\mathrm{~F}$ & $\mathrm{P}$ & 1 & $\begin{array}{l}\text { Tr, Tm, HN, } \\
\quad \text { Ner, Oc }\end{array}$ & 20 & 156 \\
\hline G. digitalis (Pouchet) Kofoid & $\mathrm{R}, \mathrm{B}$ & 5.5 & $\mathrm{~F}$ & $\mathrm{P}$ & 1 & Tm, Tr, Ner & 20 & 60 \\
\hline G. fragilis (Schütt) Kofoid & $\mathrm{R}, \mathrm{B}$ & 0.6 & $\mathrm{~F}$ & $\mathrm{P}$ & 1 & Tr, Tm, Ner, Oc & 15 & 22 \\
\hline G. fraterculus Balech & B & 15.5 & $\mathrm{~F}$ & $\mathrm{P}$ & 1 & Tm, Atl. Sur & 42 & 3066 \\
\hline G. fusiformis Graham & $\mathrm{R}$ & 0.6 & $\mathrm{~F}$ & $\mathrm{P}$ & 1 & $\mathrm{Tr}, \mathrm{Oc}$ & & \\
\hline G. grindleyi Reinecke & $\mathrm{R}, \mathrm{B}$ & 13.6 & $\mathrm{~F}$ & $\mathrm{P}$ & 1 & Tm, Atl. & 21 & 1244 \\
\hline G. hyalina Ostenfeld et J. Schmidt & $\mathrm{R}$ & 0.6 & $\mathrm{~F}$ & $\mathrm{P}$ & 1 & Tr, Ner, Oc & & \\
\hline G. pacifica Kofoid & $\mathrm{R}$ & 1.2 & $\mathrm{~F}$ & $\mathrm{P}$ & 1 & Tr, Tm, Ner, Oc & & \\
\hline G. polygramma Stein* & $\mathrm{R}, \mathrm{B}$ & 35.2 & $\mathrm{~F}$ & $\mathrm{P}$ & 1 & $\mathrm{Tr}, \mathrm{Tm}, \mathrm{Ner}$ & 10 & 2613 \\
\hline G. sphaeroidea Kofoid & $\mathrm{R}, \mathrm{B}$ & 0.9 & $\mathrm{~F}$ & $\mathrm{P}$ & 1 & $\mathrm{Tr}, \mathrm{Oc}$ & 96 & 409 \\
\hline $\begin{array}{l}\text { G. spinifera (Claparède et Lachmann) } \\
\text { Diesing* }\end{array}$ & $\mathrm{R}, \mathrm{B}$ & 16.9 & $\mathrm{~F}$ & $\mathrm{P}$ & 1 & $\mathrm{Tr}, \mathrm{Tm}, \mathrm{Ner}$ & 20 & 4960 \\
\hline G. turbynei Murray et Whitting & $\mathrm{R}, \mathrm{B}$ & 5.5 & $\mathrm{~F}$ & $\mathrm{P}$ & 1 & Tm, Ner, Oc & 10 & 190 \\
\hline G. verior Sournia & $\mathrm{B}$ & 4.9 & $\mathrm{~F}$ & $\mathrm{P}$ & 1 & Tr, Tm, Ner & 20 & 2985 \\
\hline
\end{tabular}


Apéndice 1. Continuación.

\begin{tabular}{|c|c|c|c|c|c|c|c|c|}
\hline \multirow[b]{2}{*}{ Taxa } & \multirow[b]{2}{*}{$\mathrm{M}$} & \multirow[b]{2}{*}{ Frec. } & \multirow[b]{2}{*}{$\mathrm{N}$} & \multirow[b]{2}{*}{$\mathrm{H}$} & \multirow[b]{2}{*}{ NO } & \multirow{2}{*}{$\begin{array}{l}\text { Distribución } \\
\text { Global }\end{array}$} & \multicolumn{2}{|c|}{$\begin{array}{l}\text { Abundancia } \\
\text { céls. } \mathrm{L}^{-1}\end{array}$} \\
\hline & & & & & & & Mín. & Máx. \\
\hline G. sp. & $\mathrm{B}$ & 5.8 & $\mathrm{~F}$ & $\mathrm{P}$ & 1 & Tr, Ner & 10 & 4482 \\
\hline $\begin{array}{l}\text { Greuetodinium cylindricum (Greuet) } \\
\text { Loeblich III }\end{array}$ & $\mathrm{B}, \mathrm{V}$ & 3.3 & $\mathrm{H}$ & $\mathrm{P}$ & 1 & $\mathrm{Tr}, \mathrm{Tm}, \mathrm{Oc}$ & 13 & 221 \\
\hline Gymnodinium aestuariale Hulburt & $\mathrm{B}$ & 1.8 & $\mathrm{~F}$ & $\mathrm{P}$ & 1 & $\mathrm{Tr}, \mathrm{Tm}, \mathrm{Ner}$ & 311 & 1778 \\
\hline G. allophron Larsen & B & 16.9 & $\mathrm{H}$ & $\mathrm{P}$ & 1 & Tr, Tm, Ner & 16 & 7668 \\
\hline G. attenuatum Kofoid et Swezy & $\mathrm{B}$ & 17.6 & $\mathrm{H}$ & $\mathrm{P}$ & 1 & $\mathrm{Tm}, \mathrm{Oc}$ & 32 & 137627 \\
\hline G. aureolum (Hulburt) G. Hansen & $\mathrm{B}$ & 1.8 & $\mathrm{~F}$ & $\mathrm{P}$ & 1 & Tr, Tm, Ner & 10 & 458 \\
\hline G. aureum Kofoid et Swezy & $\mathrm{B}$ & 3.0 & $\mathrm{H}$ & $\mathrm{P}$ & 1 & $\mathrm{Tm}, \mathrm{Oc}$ & 13 & 3694 \\
\hline G. catenatum Graham** & $\mathrm{R}, \mathrm{B}$ & 44.6 & $\mathrm{~F}$ & $\mathrm{P}$ & 4 & $\mathrm{Tr}, \mathrm{Tm}, \mathrm{Ner}$ & 20 & 188703 \\
\hline $\begin{array}{l}\text { G. cf. grammaticum (Pouchet) Kofoid } \\
\text { et Swezy }\end{array}$ & $\mathrm{B}$ & 1.2 & $\mathrm{~F}$ & $\mathrm{P}$ & 1 & Tm, Ner, Oc & 119 & 356 \\
\hline G. incoloratum Conrad et Kufferath & $\mathrm{B}$ & 1.2 & $\mathrm{H}$ & $\mathrm{P}$ & 1 & $\mathrm{Tr}, \mathrm{Tm}, \mathrm{Ner}$ & 20 & 3557 \\
\hline $\begin{array}{l}\text { G. instriatum (Freudenthal et Lee) } \\
\text { Coats }\end{array}$ & B & 4.9 & $\mathrm{~F}$ & $\mathrm{P}$ & 1 & $\operatorname{Tr}, \mathrm{Tm}, \mathrm{Ner}$ & 10 & 1778 \\
\hline G. parvulum Schütt & $\mathrm{B}, \mathrm{V}$ & 0.6 & $\mathrm{H}$ & $\mathrm{P}$ & 1 & Tr, Tm, Ner & 178 & 875 \\
\hline G. pygmaeum Lebour & $\mathrm{B}$ & 0.3 & $\mathrm{~F}$ & $\mathrm{P}$ & 1 & $\mathrm{Tm}, \mathrm{Oc}$ & & 4446 \\
\hline G. ravenescens Kofoid et Swezy & $\mathrm{V}$ & 0.3 & $\mathrm{H}$ & $\mathrm{P}$ & 1 & Tr, Tm, Ner & & \\
\hline G. rhomboides Schütt & $\mathrm{V}$ & 0.6 & $\mathrm{H}$ & $\mathrm{P}$ & 1 & Tr, Tm, Ner, Oc & & \\
\hline G. sphaericum Calkins & $\mathrm{V}$ & 2.1 & $\mathrm{~F}$ & $\mathrm{P}$ & 1 & Tm, Ner & & \\
\hline G. translucens Kofoid et Swezy & $\mathrm{V}$ & 0.6 & $\mathrm{H}$ & $\mathrm{P}$ & 1 & $\mathrm{Tr}, \mathrm{Tm}, \mathrm{Oc}$ & & \\
\hline G. sp. 1 & $\mathrm{~B}$ & 8.2 & $\mathrm{H}$ & $\mathrm{P}$ & 1 & Tr, Ner & 27 & 10476 \\
\hline G. sp. 2 & $\mathrm{~B}$ & 19.4 & $\mathrm{H}$ & $\mathrm{P}$ & 1 & Tr, Ner & 89 & 10607 \\
\hline G. sp. 3 & $\mathrm{~B}$ & 6.9 & $\mathrm{H}$ & $\mathrm{P}$ & 1 & Tr, Ner & 20 & 3912 \\
\hline $\begin{array}{l}\text { Gynogonadinium aequatoriale } \\
\text { Gómez }\end{array}$ & B & 0.9 & $\mathrm{~F}$ & $\mathrm{P}$ & 1 & $\mathrm{Tr}, \mathrm{Oc}$ & 50 & 2564 \\
\hline $\begin{array}{l}\text { Gyrodinium acutum (Schütt) Kofoid } \\
\text { et Swezy }\end{array}$ & $\mathrm{R}, \mathrm{B}$ & 56.4 & $\mathrm{H}$ & $\mathrm{P}$ & 1 & Tr, Ner & 10 & 7461 \\
\hline G. britannicum Kofoid et Swezy & $\mathrm{B}$ & 5.5 & $\mathrm{H}$ & $\mathrm{P}$ & 1 & Tm, Ner & 20 & 4390 \\
\hline G. falcatum Kofoid et Swezy & $\mathrm{R}, \mathrm{B}$ & 23.6 & $\mathrm{~F}$ & $\mathrm{P}$ & 1 & Tm, Tr, Ner, Oc & 10 & 1323 \\
\hline G. foliaceum Kofoid et Swezy & $\mathrm{V}$ & 11.5 & $\mathrm{~F}$ & $\mathrm{P}$ & 1 & Tm, Ner & & \\
\hline G. fusiforme Kofoid et Swezy & $\mathrm{R}, \mathrm{B}$ & 19.7 & $\mathrm{~F}$ & $\mathrm{P}$ & 1 & $\mathrm{Tr}, \mathrm{Tm}, \mathrm{Oc}$ & 27 & 8994 \\
\hline G. fusus (Meunier) Akselman & $\mathrm{R}, \mathrm{B}$ & 55.5 & $\mathrm{H}$ & $\mathrm{P}$ & 1 & Tr, Tm, Ner, Oc & 20 & 16668 \\
\hline G. cf. ochraceum Kofoid et Swezy & $\mathrm{V}$ & 0.3 & $\mathrm{H}$ & $\mathrm{P}$ & 1 & $\mathrm{Tm}, \mathrm{Oc}$ & & \\
\hline G. pepo (Schütt) Kofoid et Swezy & $\mathrm{V}$ & 0.6 & $\mathrm{H}$ & $\mathrm{P}$ & 1 & Tm, F, Ner & & \\
\hline G. spirale (Bergh) Kofoid et Swezy & $\mathrm{B}$ & 1.2 & $\mathrm{H}$ & $\mathrm{P}$ & 1 & Tm, Sbt, Ner, Oc & 20 & 653 \\
\hline G. sp. & $\mathrm{B}$ & 3.3 & $\mathrm{H}$ & $\mathrm{P}$ & 1 & Tr, Ner & 48 & 1896 \\
\hline $\begin{array}{l}\text { Heterocapsa triquetra (Ehrenberg) } \\
\text { Stein }\end{array}$ & B & 16.4 & $\mathrm{~F}$ & $\mathrm{P}$ & 1 & $\operatorname{Tr}, \mathrm{Tm}, \mathrm{Ner}$ & 47 & 46379 \\
\hline
\end{tabular}


Apéndice 1. Continuación.

\begin{tabular}{|c|c|c|c|c|c|c|c|c|}
\hline \multirow[b]{2}{*}{ Taxa } & \multirow[b]{2}{*}{$\mathrm{M}$} & \multirow[b]{2}{*}{ Frec. } & \multirow[b]{2}{*}{$\mathrm{N}$} & \multirow[b]{2}{*}{$\mathrm{H}$} & \multirow[b]{2}{*}{$\mathrm{NO}$} & \multirow{2}{*}{$\begin{array}{l}\text { Distribución } \\
\text { Global }\end{array}$} & \multicolumn{2}{|c|}{$\begin{array}{c}\text { Abundancia } \\
\text { céls. } \mathrm{L}^{-1}\end{array}$} \\
\hline & & & & & & & Mín. & Máx. \\
\hline $\begin{array}{l}\text { Heterodinium blackmanii (Murray et } \\
\text { Witting) Kofoid }\end{array}$ & $\mathrm{R}$ & 0.3 & $\mathrm{H}$ & $\mathrm{P}$ & 1 & $\mathrm{Tr}, \mathrm{Oc}$ & & \\
\hline H. sp. & B & 0.3 & $\mathrm{H}$ & $\mathrm{P}$ & 1 & $\mathrm{Tr}, \mathrm{Oc}$ & & 158 \\
\hline $\begin{array}{l}\text { Histioneis hyalina Kofoid et } \\
\text { Michener }\end{array}$ & $\mathrm{R}, \mathrm{B}$ & 0.6 & $\mathrm{~F}$ & $\mathrm{P}$ & 1 & $\mathrm{Tr}, \mathrm{Oc}$ & 12 & 35 \\
\hline H. reticulata Kofoid & $\mathrm{B}$ & 0.3 & $\mathrm{~F}$ & $\mathrm{P}$ & 1 & Tr, Ner & & 327 \\
\hline $\begin{array}{l}\text { Karenia asteriochroma De Salas, } \\
\text { Bolch et Hallegraeff** }\end{array}$ & B & 9.4 & $\mathrm{~F}$ & $\mathrm{P}$ & 1 & Tm, Ner & 10 & 2818 \\
\hline $\begin{array}{l}\text { K. bicuneiformis Botes, Sym et } \\
\text { Pritcher** }\end{array}$ & $\mathrm{B}$ & 6.7 & $\mathrm{~F}$ & $\mathrm{P}$ & 1 & Sbt, Ner & 20 & 4572 \\
\hline $\begin{array}{l}\text { K. cf. brevis (Davis) Hansen et } \\
\text { Moestrup** }\end{array}$ & $\mathrm{R}, \mathrm{B}$ & 8.5 & $\mathrm{~F}$ & $\mathrm{P}$ & 1 & $\mathrm{Tr}, \mathrm{Tm}, \mathrm{Ner}$ & 20 & 4555 \\
\hline $\begin{array}{l}\text { K. brevisulcata (Chang) Hansen et } \\
\text { Moestrup** }\end{array}$ & $\mathrm{B}$ & 7.3 & $\mathrm{~F}$ & $\mathrm{P}$ & 1 & Tm, Tr, Ner & 20 & 1541 \\
\hline $\begin{array}{l}\text { K. mikimotoi (Miyake et Kominami } \\
\text { ex Oda) Hansen et Moestrup** }\end{array}$ & $\mathrm{B}, \mathrm{V}$ & 3.0 & $\mathrm{~F}$ & $\mathrm{P}$ & 1 & Tm, Ner, Oc & 10 & 1750 \\
\hline $\begin{array}{l}\text { K. papillonacea } \text { Haywood et } \\
\text { Steidinger* }\end{array}$ & $\mathrm{B}$ & 1.8 & $\mathrm{~F}$ & $\mathrm{P}$ & 1 & Tr, Tm, Ner, Oc & 20 & 491 \\
\hline $\begin{array}{l}\text { K. selliformis Haywood, Steidinger et } \\
\text { McKenzie** }\end{array}$ & $\mathrm{B}$ & 11.8 & $\mathrm{~F}$ & $\mathrm{P}$ & 1 & $\mathrm{Tr}, \mathrm{Tm}, \mathrm{Ner}$ & 39 & 9431 \\
\hline$K . \mathrm{sp}$. & B & 7.9 & $\mathrm{~F}$ & $\mathrm{P}$ & 1 & Tr, Ner & 10 & 356 \\
\hline $\begin{array}{l}\text { Katodinium glaucum (Lebour) } \\
\text { Loeblich III }\end{array}$ & $\mathrm{B}$ & 4.9 & $\mathrm{~F}$ & $\mathrm{P}$ & 1 & Tr, Tm, Ner & 48 & 4025 \\
\hline $\begin{array}{l}\text { Kofoidinium lebourae (Pavillard) } \\
\text { F.J.R. Taylor }\end{array}$ & $\mathrm{R}$ & 1.8 & $\mathrm{H}$ & $\mathrm{P}$ & 1 & $\mathrm{Tm}, \mathrm{Tr}, \mathrm{Ner}, \mathrm{Oc}$ & & \\
\hline $\begin{array}{l}\text { K. splendens J. Cachon et } \mathrm{M} \text {. } \\
\text { Cachon }\end{array}$ & $\mathrm{B}$ & 0.3 & $\mathrm{H}$ & $\mathrm{P}$ & 1 & $\mathrm{Tr}, \mathrm{Tm}, \mathrm{Oc}$ & & 20 \\
\hline K. velelloides Pavillard & $\mathrm{R}, \mathrm{B}$ & 5.2 & $\mathrm{H}$ & $\mathrm{P}$ & 1 & $\mathrm{Tm}, \mathrm{Tr}, \mathrm{Oc}$ & 20 & 4195 \\
\hline $\begin{array}{l}\text { Lingulodinium polyedra (Stein) } \\
\text { Dodge** }\end{array}$ & $\mathrm{R}, \mathrm{B}$ & 20.0 & $\mathrm{~F}$ & $\mathrm{P}$ & 1 & $\mathrm{Tr}, \mathrm{Tm}, \mathrm{Ner}, \mathrm{Oc}$ & 18 & 13074 \\
\hline Metaphalacroma skogsbergii Tai & $\mathrm{B}$ & 0.9 & M & $\mathrm{P}$ & 1 & $\mathrm{Tm}, \mathrm{Tr}, \mathrm{Oc}$ & 42 & 118 \\
\hline $\begin{array}{l}\text { Nematodinium torpedo Kofoid et } \\
\text { Swezy }\end{array}$ & B & 9.7 & $\mathrm{H}$ & $\mathrm{P}$ & 1 & Tm, Tr, Ner, Oc & 32 & 4195 \\
\hline $\begin{array}{l}\text { Neoceratium arietinum (Cleve) } \\
\text { Gómez, Moreira et López-García }\end{array}$ & $\mathrm{R}$ & 0.3 & M & $\mathrm{P}$ & 1 & $\mathrm{Tm}, \mathrm{Tr}, \mathrm{Ner}, \mathrm{Oc}$ & & \\
\hline $\begin{array}{l}\text { N. axiale (Kofoid) Gómez, Moreira et } \\
\text { López-García }\end{array}$ & $\mathrm{R}$ & 0.3 & $\mathrm{M}$ & $\mathrm{P}$ & 1 & Tr, Ner, Oc & & \\
\hline
\end{tabular}


Apéndice 1. Continuación.

\begin{tabular}{|c|c|c|c|c|c|c|c|c|}
\hline \multirow[b]{2}{*}{ Taxa } & \multirow[b]{2}{*}{ M } & \multirow[b]{2}{*}{ Frec. } & \multirow[b]{2}{*}{$\mathrm{N}$} & \multirow[b]{2}{*}{$\mathrm{H}$} & \multirow[b]{2}{*}{ NO } & \multirow{2}{*}{$\begin{array}{l}\text { Distribución } \\
\text { Global }\end{array}$} & \multicolumn{2}{|c|}{$\begin{array}{l}\text { Abundancia } \\
\text { céls. } L^{-1}\end{array}$} \\
\hline & & & & & & & Mín. & Máx. \\
\hline $\begin{array}{l}\text { N. azoricum (Cleve) Gómez, Moreira } \\
\text { et López-García }\end{array}$ & $\mathrm{R}$ & 0.6 & M & $\mathrm{P}$ & 1 & $\mathrm{Tm}, \mathrm{Tr}, \mathrm{Ner}, \mathrm{Oc}$ & & \\
\hline $\begin{array}{l}\text { N. balechii (Meave, Okolodkov } \\
\text { et Zamudio) Gómez, Moreira et } \\
\text { López-García }\end{array}$ & $\mathrm{R}, \mathrm{B}$ & 33.9 & M & $\mathrm{P}$ & 4 & $\mathrm{Tm}, \mathrm{Tr}, \mathrm{Ner}, \mathrm{Oc}$ & 18 & 2516 \\
\hline $\begin{array}{l}\text { Neoceratium (Ceratium) balechii } \mathrm{f} . \\
\text { longum Zamudio et Meave }\end{array}$ & $\mathrm{R}, \mathrm{B}$ & 4.2 & M & $\mathrm{P}$ & 4 & $\mathrm{Tm}, \mathrm{Tr}, \mathrm{Ner}, \mathrm{Oc}$ & 96 & 351 \\
\hline $\begin{array}{l}\text { N. belone (Cleve) Gómez, Moreira et } \\
\text { López-García }\end{array}$ & $\mathrm{R}, \mathrm{B}$ & 1.8 & M & $\mathrm{P}$ & 1 & $\mathrm{Tr}, \mathrm{Oc}$ & 112 & 278 \\
\hline $\begin{array}{l}\text { N. biceps (Claparède et Lachmann) } \\
\text { Gómez, Moreira et López-García }\end{array}$ & $\mathrm{R}$ & 0.6 & M & $\mathrm{P}$ & 1 & Tr, Ind. & & \\
\hline $\begin{array}{l}\text { N. breve (Ostenfeld et .J. Schmidt) } \\
\text { Gómez, Moreira et López-García } \\
\text { var. breve }\end{array}$ & $\mathrm{R}, \mathrm{B}$ & 30.3 & M & $\mathrm{P}$ & 4 & Tr, Ner, Oc & 10 & 1041 \\
\hline $\begin{array}{l}\text { Neoceratium (Ceratium) breve var. } \\
\text { parallelum (J. Schmidt) Jörgensen }\end{array}$ & $\mathrm{R}, \mathrm{B}$ & 7.3 & M & $\mathrm{P}$ & 4 & Tr, Ner, Oc & 24 & 4394 \\
\hline $\begin{array}{l}\text { Neoceratium (Ceratium)breve var. } \\
\text { schmidtii (Jörgensen) Sournia }\end{array}$ & $\mathrm{R}, \mathrm{B}$ & 12.1 & M & $\mathrm{P}$ & 1 & $\mathrm{Tm}, \mathrm{Tr}, \mathrm{Oc}$ & 46 & 2760 \\
\hline $\begin{array}{l}\text { N. candelabrum (Ehrenberg) Gómez, } \\
\text { Moreira et López-García }\end{array}$ & $\mathrm{R}, \mathrm{B}$ & 13.3 & M & $\mathrm{P}$ & 4 & $\mathrm{Tm}, \mathrm{Tr}, \mathrm{Ner}, \mathrm{Oc}$ & 28 & 567 \\
\hline $\begin{array}{l}\text { N. carriense (Gourret) Gómez, Moreira } \\
\text { et López-García var. carriense }\end{array}$ & $\mathrm{R}, \mathrm{B}$ & 11.2 & M & $\mathrm{P}$ & 1 & Tr, Ner, Oc & 18 & 57 \\
\hline $\begin{array}{l}\text { Neoceratium (Ceratium) carriense } \\
\text { var. volans (Cleve) Jörgensen }\end{array}$ & $\mathrm{R}$ & 0.3 & M & $\mathrm{P}$ & 1 & Tr, Ner, Oc & & \\
\hline $\begin{array}{l}\text { N. contortum (Gourret) Gómez, } \\
\text { Moreira et López-García var. } \\
\text { contortum }\end{array}$ & $\mathrm{R}$ & 5.8 & M & $\mathrm{P}$ & 1 & Tr, Ner, Oc & & \\
\hline $\begin{array}{l}\text { Neoceratium (Ceratium) contortum } \\
\text { var. karstenii (Pavillard) Sournia }\end{array}$ & $\mathrm{R}$ & 0.6 & M & $\mathrm{P}$ & 1 & $\mathrm{Tm}, \mathrm{Tr}, \mathrm{Ner}, \mathrm{Oc}$ & & \\
\hline $\begin{array}{l}\text { Neoceratium (Ceratium) contortum } \\
\text { var. robustum (Karsten) Sournia }\end{array}$ & $\mathrm{R}, \mathrm{B}$ & 1.5 & M & $\mathrm{P}$ & 1 & Tr, Ner, Oc & 10 & 40 \\
\hline $\begin{array}{l}\text { N. contrarium (Gourret) Gómez, } \\
\text { Moreira et López-García }\end{array}$ & $\mathrm{R}, \mathrm{B}$ & 10.6 & M & $\mathrm{P}$ & 1 & $\mathrm{Tr}, \mathrm{Tm}, \mathrm{Ner}, \mathrm{Oc}$ & 20 & 40 \\
\hline $\begin{array}{l}\text { N. declinatum (Karsten) Gómez, } \\
\text { Moreira et López-García }\end{array}$ & $\mathrm{R}, \mathrm{B}$ & 5.5 & M & $\mathrm{P}$ & 1 & Tr, Tm, Ner, Oc & 37 & 474 \\
\hline $\begin{array}{l}\text { Neoceratium (Ceratium) declinatum } \mathrm{f} \text {. } \\
\text { normale Jörgensen }\end{array}$ & $\mathrm{R}$ & 0.3 & M & $\mathrm{P}$ & 1 & Tr, Sbt, Ner, Oc & & \\
\hline
\end{tabular}


Apéndice 1. Continuación.

\begin{tabular}{|c|c|c|c|c|c|c|c|c|}
\hline \multirow[b]{2}{*}{ Taxa } & \multirow[b]{2}{*}{ M } & \multirow[b]{2}{*}{ Frec. } & \multirow[b]{2}{*}{$\mathrm{N}$} & \multirow[b]{2}{*}{$\mathrm{H}$} & \multirow[b]{2}{*}{$\mathrm{NO}$} & \multirow{2}{*}{$\begin{array}{l}\text { Distribución } \\
\text { Global }\end{array}$} & \multicolumn{2}{|c|}{$\begin{array}{c}\text { Abundancia } \\
\text { céls. } \mathrm{L}^{-1}\end{array}$} \\
\hline & & & & & & & Mín. & Máx. \\
\hline $\begin{array}{l}\text { N. deflexum (Kofoid) Gómez, } \\
\text { Moreira et López-García }\end{array}$ & $\mathrm{R}, \mathrm{B}$ & 6.7 & $\mathrm{M}$ & $\mathrm{P}$ & 1 & Tr, Ner, Oc & 18 & 106 \\
\hline $\begin{array}{l}\text { N. dens (Ostenfeld et Schmidt) } \\
\text { Gómez, Moreira et López-García }\end{array}$ & $\mathrm{R}, \mathrm{B}$ & 7.6 & M & $\mathrm{P}$ & 4 & Tr, Ind.-Pac. & 18 & 1462 \\
\hline $\begin{array}{l}\text { N. digitatum (Schütt) Gómez, Moreira } \\
\text { et López-García }\end{array}$ & $\mathrm{R}, \mathrm{B}$ & 1.8 & M & $\mathrm{P}$ & 1 & $\mathrm{Tm}, \mathrm{Tr}, \mathrm{Ner}, \mathrm{Oc}$ & 27 & 96 \\
\hline $\begin{array}{l}\text { N. euarcuatum (Jörgensen) Gómez, } \\
\text { Moreira et López-García }\end{array}$ & $\mathrm{R}, \mathrm{B}$ & 5.2 & M & $\mathrm{P}$ & 1 & Tr, Ner, Oc & 80 & 109 \\
\hline $\begin{array}{l}\text { N. extensum (Gourret) Gómez, } \\
\text { Moreira et López-García }\end{array}$ & $\mathrm{R}, \mathrm{B}$ & 16.4 & M & $\mathrm{P}$ & 1 & $\mathrm{Tr}$, Ner, Oc & 10 & 664 \\
\hline $\begin{array}{l}\text { N. falcatiforme (Jörgensen) Gómez, } \\
\text { Moreira et López-García }\end{array}$ & $\mathrm{R}, \mathrm{B}$ & 7.3 & M & $\mathrm{P}$ & 1 & Tr, Sbt, Ner, Oc & 18 & 50 \\
\hline $\begin{array}{l}\text { N. falcatum (Kofoid) Gómez, Moreira } \\
\text { et López-García }\end{array}$ & $\mathrm{R}, \mathrm{B}$ & 11.5 & M & $\mathrm{P}$ & 1 & Tr, Ner, Oc & 35 & 4049 \\
\hline $\begin{array}{l}\text { N. furca (Ehrenberg) Gómez, Moreira } \\
\text { et López-García var. furca* }\end{array}$ & $\mathrm{R}, \mathrm{B}$ & 78.8 & M & $\mathrm{P}$ & 4 & Tm, Ner, Oc & 10 & 6856 \\
\hline $\begin{array}{l}\text { Neoceratium (Ceratium)furca var. } \\
\text { eugrammum (Ehrenberg) Schiller }\end{array}$ & $\mathrm{R}, \mathrm{B}$ & 59.1 & M & $\mathrm{P}$ & 4 & $\mathrm{Tr}, \mathrm{Tm}, \mathrm{Ner}, \mathrm{Oc}$ & 10 & 4102 \\
\hline $\begin{array}{l}\text { Neoceratium (Ceratium)furca var. } \\
\text { hircus (Schröder) Margalef ex } \\
\text { Sournia }\end{array}$ & $\mathrm{R}$ & 0.6 & M & $\mathrm{P}$ & 1 & Tr, Tm, Ner, Oc & & \\
\hline $\begin{array}{l}\text { N. fusus (Ehrenberg) Gómez, Moreira } \\
\text { et López-García var. fusus* }\end{array}$ & $\mathrm{R}, \mathrm{B}$ & 58.2 & M & $\mathrm{P}$ & 1 & Tr, Tm, Ner, Oc & 10 & 3102 \\
\hline $\begin{array}{l}\text { Neoceratium (Ceratium)fusus var. } \\
\text { seta (Ehrenberg) Sournia }\end{array}$ & $\mathrm{R}$ & 6.4 & M & $\mathrm{P}$ & 1 & Tr, Tm, Ner, Oc & & \\
\hline $\begin{array}{l}\text { N. gibberum (Gourret) Gómez, Moreira } \\
\text { et López-García var. gibberum }\end{array}$ & $\mathrm{R}, \mathrm{B}$ & 6.4 & M & $\mathrm{P}$ & 1 & Tr, Ner, Oc & 20 & 84 \\
\hline $\begin{array}{l}\text { Neoceratium (Ceratium)gibberum } \\
\text { var. dispar (Pouchet) Sournia }\end{array}$ & $\mathrm{R}$ & 2.1 & M & $\mathrm{P}$ & 1 & $\mathrm{Tr}$, Ner, Oc & & \\
\hline $\begin{array}{l}\text { Neoceratium (Ceratium)gibberum } \\
\text { var. subaequale Jörgensen }\end{array}$ & $\mathrm{R}$ & 2.7 & M & $\mathrm{P}$ & 1 & $\mathrm{Tr}$, Ner, Oc & & \\
\hline $\begin{array}{l}\text { N. gravidum (Gourret) Gómez, } \\
\text { Moreira et López-García var. } \\
\text { gravidum }\end{array}$ & $\mathrm{R}$ & 0.6 & M & $\mathrm{P}$ & 1 & Tr, Ner, Oc & & \\
\hline $\begin{array}{l}\text { Neoceratium (Ceratium)gravidum } \\
\text { var. elongata Wood }\end{array}$ & $\mathrm{R}$ & 0.3 & M & $\mathrm{P}$ & 1 & $\mathrm{Tr}$, Ner, Oc & & \\
\hline $\begin{array}{l}\text { N. hexacanthum (Gourret) Gómez, } \\
\text { Moreira et López-García }\end{array}$ & $\mathrm{R}$ & 1.2 & M & $\mathrm{P}$ & 4 & Tr, Sbt, Ner, Oc & & \\
\hline
\end{tabular}


Apéndice 1. Continuación.

\begin{tabular}{|c|c|c|c|c|c|c|c|c|}
\hline \multirow[b]{2}{*}{ Таха } & \multirow[b]{2}{*}{$\mathrm{M}$} & \multirow[b]{2}{*}{ Frec. } & \multirow[b]{2}{*}{$\mathrm{N}$} & \multirow[b]{2}{*}{$\mathrm{H}$} & \multirow[b]{2}{*}{$\mathrm{NO}$} & \multirow{2}{*}{$\begin{array}{l}\text { Distribución } \\
\text { Global }\end{array}$} & \multicolumn{2}{|c|}{$\begin{array}{c}\text { Abundancia } \\
\text { céls. } \mathrm{L}^{-1}\end{array}$} \\
\hline & & & & & & & Mín. & Máx. \\
\hline $\begin{array}{l}\text { N. horridum (Gran) Gómez, Moreira } \\
\text { et López-García var. horridum }\end{array}$ & $\mathrm{R}, \mathrm{B}$ & 6.4 & $\mathrm{M}$ & $\mathrm{P}$ & 1 & $\mathrm{Tm}, \mathrm{Sbt}, \mathrm{Oc}$ & 20 & 1124 \\
\hline $\begin{array}{l}\text { Neoceratium (Ceratium)horridum } \\
\text { var. molle Böhm }\end{array}$ & $\mathrm{R}, \mathrm{B}$ & 0.9 & M & $\mathrm{P}$ & 1 & Tm, Tr, Ner, Oc & 12 & 67 \\
\hline $\begin{array}{l}N \text {. inflatum (Kofoid) Gómez, Moreira } \\
\text { et López-García }\end{array}$ & $\mathrm{R}, \mathrm{B}$ & 0.6 & M & $\mathrm{P}$ & 1 & Tm, Tr, Ner, Oc & 10 & 20 \\
\hline $\begin{array}{l}\text { N. kofoidii (Jörgensen) Gómez, } \\
\text { Moreira et López-García }\end{array}$ & $\mathrm{R}, \mathrm{B}$ & 17.6 & M & $\mathrm{P}$ & 1 & $\mathrm{Tr}, \mathrm{Tm}, \mathrm{Ner}, \mathrm{Oc}$ & 20 & 1960 \\
\hline $\begin{array}{l}\text { N. lineatum (Ehrenberg) Gómez, } \\
\text { Moreira et López-García }\end{array}$ & $\mathrm{R}, \mathrm{B}$ & 2.1 & M & $\mathrm{P}$ & 1 & Tr, Atl. norte & 28 & 977 \\
\hline $\begin{array}{l}\text { N. longirostrum (Gourret) Gómez, } \\
\text { Moreira et López-García }\end{array}$ & $\mathrm{R}, \mathrm{B}$ & 1.5 & M & $\mathrm{P}$ & 1 & Tr, Sbt, Ner, Oc & 10 & 20 \\
\hline $\begin{array}{l}\text { N. longissimum (Schröder) Gómez, } \\
\text { Moreira et López-García }\end{array}$ & $\mathrm{R}$ & 0.3 & $\mathrm{M}$ & $\mathrm{P}$ & 1 & Tm, Tr, Ner, Oc & & 20 \\
\hline $\begin{array}{l}\text { N. lunula (Schimper ex Karsten) } \\
\text { Gómez, Moreira et López-García }\end{array}$ & $\mathrm{R}, \mathrm{B}$ & 3.0 & M & $\mathrm{P}$ & 4 & Tr, Sbt, Ner, Oc & 10 & 59 \\
\hline $\begin{array}{l}\text { Neoceratium (Ceratium)lunula var. } \\
\text { robustum F.J.R. Taylo }\end{array}$ & $\mathrm{R}$ & 0.6 & M & $\mathrm{P}$ & 4 & Tr, Sbt, Ner, Oc & & \\
\hline $\begin{array}{l}\text { N. macroceros F.J.R. Taylo var. } \\
\text { macroceros }\end{array}$ & $\mathrm{R}, \mathrm{B}$ & 15.5 & M & $\mathrm{P}$ & 4 & Tr, Ner, Oc & 20 & 558 \\
\hline $\begin{array}{l}\text { Neoceratium (Ceratium)macroceros } \\
\text { var. gallicum (Kofoid) Sournia }\end{array}$ & $\mathrm{R}$ & 0.6 & M & $\mathrm{P}$ & 4 & Tr, Ner, Oc & & \\
\hline $\begin{array}{l}\text { N. massiliense (Gourret) Gómez, Moreira } \\
\text { et López-García var. massiliense }\end{array}$ & $\mathrm{R}, \mathrm{B}$ & 23.9 & M & $\mathrm{P}$ & 1 & Tm, Tr, Ner, Oc & 20 & 1069 \\
\hline $\begin{array}{l}\text { Neoceratium (Ceratium) massiliense } \\
\text { var. armatum (Karsten) Jörgensen }\end{array}$ & $\mathrm{R}$ & 2.4 & M & $\mathrm{P}$ & 1 & Tm, Tr, Ner & & \\
\hline $\begin{array}{l}\text { N. minutum (Jörgensen) Gómez, } \\
\text { Moreira et López-García }\end{array}$ & $\mathrm{R}, \mathrm{B}$ & 4.2 & M & $\mathrm{P}$ & 1 & Tr, Tm, Ner, Oc & 16 & 1474 \\
\hline $\begin{array}{l}\text { N. pentagonum (Gourret) Gómez, } \\
\text { Moreira et López-García }\end{array}$ & $\mathrm{R}, \mathrm{B}$ & 3.0 & M & $\mathrm{P}$ & 4 & Tr, Sbt, Ner, Oc & 12 & 43 \\
\hline $\begin{array}{l}\text { N. praelongum (Lemmermann) } \\
\text { Gómez, Moreira et López-García }\end{array}$ & $\mathrm{R}$ & 1.2 & M & $\mathrm{P}$ & 1 & Tr, Ner, Oc & & \\
\hline $\begin{array}{l}\text { N. ranipes (Cleve) Gómez, Moreira et } \\
\text { López-García }\end{array}$ & $\mathrm{R}$ & 1.2 & M & $\mathrm{P}$ & 1 & Tr, Sbt, Ner, Oc & & \\
\hline $\begin{array}{l}\text { N. reflexum (Cleve) Gómez, Moreira } \\
\text { et López-García }\end{array}$ & $\mathrm{R}$ & 2.1 & M & $\mathrm{P}$ & 1 & $\mathrm{Tr}, \mathrm{Ner}, \mathrm{Oc}$ & & \\
\hline $\begin{array}{l}\text { N. setaceum (Jörgensen) Gómez, } \\
\text { Moreira et López-García }\end{array}$ & $\mathrm{R}$ & 0.3 & M & $\mathrm{P}$ & 1 & Tr, Ner, Oc & & \\
\hline
\end{tabular}


Apéndice 1. Continuación.

\begin{tabular}{|c|c|c|c|c|c|c|c|c|}
\hline \multirow[b]{2}{*}{ Taxa } & \multirow[b]{2}{*}{$\mathrm{M}$} & \multirow[b]{2}{*}{ Frec. } & \multirow[b]{2}{*}{$\mathrm{N}$} & \multirow[b]{2}{*}{$\mathrm{H}$} & \multirow[b]{2}{*}{ NO } & \multirow{2}{*}{$\begin{array}{l}\text { Distribución } \\
\text { Global }\end{array}$} & \multicolumn{2}{|c|}{$\begin{array}{c}\text { Abundancia } \\
\text { céls. } \mathrm{L}^{-1}\end{array}$} \\
\hline & & & & & & & Mín. & Máx. \\
\hline $\begin{array}{l}\text { N. symetricum (Pavillard) Gómez, } \\
\text { Moreira et López-García var. } \\
\text { symetricum }\end{array}$ & $\mathrm{R}$ & 4.2 & $\mathrm{M}$ & $\mathrm{P}$ & 1 & $\mathrm{Tr}, \mathrm{Ner}, \mathrm{Oc}$ & & \\
\hline $\begin{array}{l}\text { Neoceratium (Ceratium)symetricum } \\
\text { var. coarctatum (Pavillard) Graham } \\
\text { et Bronikovsky }\end{array}$ & $\mathrm{R}$ & 0.6 & M & $\mathrm{P}$ & 1 & $\mathrm{Tm}, \mathrm{Tr}, \mathrm{Ner}, \mathrm{Oc}$ & & \\
\hline $\begin{array}{l}\text { N. tenue (Ostenfeld et J. Schmidt) } \\
\text { Gómez, Moreira et López-García }\end{array}$ & $\mathrm{R}$ & 0.6 & M & $\mathrm{P}$ & 1 & Tm, Ner, Oc & & \\
\hline $\begin{array}{l}\text { N. teres (Kofoid) Gómez, Moreira et } \\
\text { López-García }\end{array}$ & $\mathrm{R}, \mathrm{B}$ & 6.4 & M & $\mathrm{P}$ & 1 & Tr, Sbt, Ner, Oc & 10 & 20 \\
\hline $\begin{array}{l}\text { N. trichoceros (Ehrenberg) Gómez, } \\
\text { Moreira et López-García }\end{array}$ & $\mathrm{R}, \mathrm{B}$ & 25.2 & M & $\mathrm{P}$ & 1 & $\mathrm{Tm}, \mathrm{Tr}, \mathrm{Ner}, \mathrm{Oc}$ & 20 & 779 \\
\hline $\begin{array}{l}\text { N. tripos (Ehrenberg) Gómez, } \\
\text { Moreira et López-García var. } \\
\text { tripos* }\end{array}$ & $\mathrm{R}, \mathrm{B}$ & 22.7 & M & $\mathrm{P}$ & 1 & Tm, Ner, Oc & 20 & 653 \\
\hline $\begin{array}{l}\text { Neoceratium (Ceratium)tripos var. } \\
\text { atlanticum (Ostenfeld) Paulsen }\end{array}$ & $\mathrm{R}, \mathrm{B}$ & 2.1 & M & $\mathrm{P}$ & 1 & $\mathrm{Tm}, \mathrm{Tr}, \mathrm{Ner}, \mathrm{Oc}$ & 15 & 20 \\
\hline $\begin{array}{l}\text { Neoceratium (Ceratium)tripos var. } \\
\text { pulchellum Schröder }\end{array}$ & $\mathrm{R}$ & 1.5 & M & $\mathrm{P}$ & 1 & $\mathrm{Tr}, \mathrm{Tm}, \mathrm{Ner}, \mathrm{Oc}$ & & \\
\hline $\begin{array}{l}\text { N. vultur (Cleve) Gómez, Moreira et } \\
\text { López-García }\end{array}$ & $\mathrm{R}, \mathrm{B}$ & 5.5 & M & $\mathrm{P}$ & 4 & Tr, Sbt, Ner, Oc & 571 & 761 \\
\hline $\begin{array}{c}\text { Neoceratium (Ceratium)vultur f. } \\
\text { japonicum (Schröder) Wood }\end{array}$ & $\mathrm{R}$ & 4.6 & M & $\mathrm{P}$ & 4 & $\mathrm{Tr}, \mathrm{Ner}, \mathrm{Oc}$ & & \\
\hline $\begin{array}{l}\text { Neoceratium (Ceratium) vultur f. } \\
\text { recurvum (Jörgensen) Schiller }\end{array}$ & $\mathrm{R}$ & 0.9 & M & $\mathrm{P}$ & 4 & Tr, Ner, Oc & & \\
\hline $\begin{array}{l}\text { Neoceratium (Ceratium)vultur } \mathrm{f} . \\
\text { sumatranum (Karsten) Sournia }\end{array}$ & $\mathrm{R}$ & 0.6 & M & $\mathrm{P}$ & 4 & Tr, Sbt, Ner, Oc & & \\
\hline Neoceratium sp. & B & 1.2 & M & $\mathrm{P}$ & 1 & Tr, Ner, & 20 & 193 \\
\hline $\begin{array}{l}\text { Noctiluca scintillans (Macartney) } \\
\text { Kofoid et Swezy* }\end{array}$ & $\mathrm{R}, \mathrm{B}$ & 11.2 & $\mathrm{H}$ & $\mathrm{P}$ & 1 & Tm, Ner & 32 & 3144 \\
\hline Oodinium sp. & B & 0.3 & $\mathrm{H}$ & $\mathrm{P}$ & 2 & Tm, Ner & 20 & 108 \\
\hline Ornithocercus galea (Pouchet) Abé & $\mathrm{R}, \mathrm{B}$ & 3.9 & $\mathrm{~F}$ & $\mathrm{P}$ & 1 & $\mathrm{Tm}, \mathrm{Tr}, \mathrm{Oc}$ & 15 & 30 \\
\hline O. heteroporus Kofoid & $\mathrm{R}, \mathrm{B}$ & 3.0 & $\mathrm{~F}$ & $\mathrm{P}$ & 1 & $\mathrm{Tm}, \operatorname{Tr}$ & 20 & 43 \\
\hline O. magnificus Stein & $\mathrm{R}, \mathrm{B}$ & 19.7 & $\mathrm{~F}$ & $\mathrm{P}$ & 1 & $\mathrm{Tm}, \operatorname{Tr}$ & 10 & 122 \\
\hline O. orbiculatus Kofoid et Michener & $\mathrm{R}$ & 0.3 & $\mathrm{~F}$ & $\mathrm{P}$ & 1 & $\mathrm{Tr}, \mathrm{Oc}$ & & \\
\hline O. quadratus Schütt & $\mathrm{R}$ & 4.2 & $\mathrm{~F}$ & $\mathrm{P}$ & 1 & $\mathrm{Tm}, \operatorname{Tr}$ & & \\
\hline O. splendidus Schütt & $\mathrm{R}$ & 0.3 & $\mathrm{~F}$ & $\mathrm{P}$ & 1 & $\mathrm{Tm}, \operatorname{Tr}$ & & \\
\hline O. steinii Schütt & $\mathrm{R}, \mathrm{B}$ & 12.1 & $\mathrm{~F}$ & $\mathrm{P}$ & 1 & $\mathrm{Tr}, \mathrm{Ner}$ & 40 & 474 \\
\hline
\end{tabular}


Apéndice 1. Continuación.

\begin{tabular}{|c|c|c|c|c|c|c|c|c|}
\hline \multirow[b]{2}{*}{ Taxa } & \multirow[b]{2}{*}{ M } & \multirow[b]{2}{*}{ Frec. } & \multirow[b]{2}{*}{$\mathrm{N}$} & \multirow[b]{2}{*}{$\mathrm{H}$} & \multirow[b]{2}{*}{ NO } & \multirow{2}{*}{$\begin{array}{c}\text { Distribución } \\
\text { Global }\end{array}$} & \multicolumn{2}{|c|}{$\begin{array}{c}\text { Abundancia } \\
\text { céls. } \mathrm{L}^{-1}\end{array}$} \\
\hline & & & & & & & Mín. & Máx. \\
\hline $\begin{array}{l}\text { O. thumii (A. Schmidt) Kofoid et } \\
\text { Skogsberg }\end{array}$ & $\mathrm{R}, \mathrm{B}$ & 16.7 & $\mathrm{~F}$ & $\mathrm{P}$ & 1 & $\mathrm{Tr}, \mathrm{Sbt}$ & 20 & 88876 \\
\hline Oxyphysis oxytoxoides Kofoid & $\mathrm{B}$ & 1.2 & $\mathrm{H}$ & $\mathrm{P}$ & 1 & $\mathrm{Tm}, \mathrm{Tr}, \mathrm{Ner}$ & 40 & 779 \\
\hline Oxytoxum caudatum Schiller & $\mathrm{B}$ & 2.7 & $\mathrm{~F}$ & $\mathrm{P}$ & 1 & $\mathrm{Tm}, \mathrm{Oc}$ & 20 & 2516 \\
\hline O. laticeps Schiller & B & 0.9 & $\mathrm{~F}$ & $\mathrm{P}$ & 1 & Tm, Ner, Oc & 20 & 175 \\
\hline O. nanum Halldal & $\mathrm{B}$ & 0.9 & $\mathrm{~F}$ & $\mathrm{P}$ & 1 & $\mathrm{Tr}, \mathrm{Tm}, \mathrm{Ner}, \mathrm{Oc}$ & 40 & 356 \\
\hline O. ovale Schiller & $\mathrm{B}$ & 1.2 & $\mathrm{~F}$ & $\mathrm{P}$ & 1 & $\mathrm{Tm}, \mathrm{Oc}$ & 80 & 2406 \\
\hline O. parvum Schiller & $\mathrm{B}$ & 0.3 & $\mathrm{~F}$ & $\mathrm{P}$ & 1 & $\mathrm{Tm}, \mathrm{Tr}, \mathrm{Oc}$ & & 20 \\
\hline O. sceptrum (Stein) Schröder & $\mathrm{R}, \mathrm{B}$ & 4.6 & $\mathrm{~F}$ & $\mathrm{P}$ & 1 & $\mathrm{Tm}, \mathrm{Tr}, \mathrm{Oc}$ & 20 & 499 \\
\hline O. scolopax Stein & $\mathrm{R}, \mathrm{B}$ & 3.9 & $\mathrm{~F}$ & $\mathrm{P}$ & 1 & $\mathrm{Tr}, \mathrm{Tm}, \mathrm{Oc}$ & 10 & 1477 \\
\hline O. variabile Schiller & $\mathrm{R}$ & 0.3 & $\mathrm{~F}$ & $\mathrm{P}$ & 1 & $\mathrm{Tr}, \mathrm{Sbt}, \mathrm{Oc}$ & & \\
\hline$O$. sp. 1 & $\mathrm{~B}$ & 7.9 & $\mathrm{~F}$ & $\mathrm{P}$ & 1 & $\mathrm{Tr}, \mathrm{Oc}$ & 10 & 3144 \\
\hline O. sp. 2 & $\mathrm{~B}$ & 0.3 & $\mathrm{~F}$ & $\mathrm{P}$ & 1 & $\mathrm{Tr}, \mathrm{Oc}$ & & 30 \\
\hline $\begin{array}{l}\text { Peridiniella danica (Paulsen) } \\
\text { Okolodkov et Dodge }\end{array}$ & $\mathrm{R}$ & 0.3 & $\mathrm{~F}$ & $\mathrm{P}$ & 1 & Tm, Tr, Ner & & \\
\hline Peridinium quinquecorne Abé* & $\mathrm{R}, \mathrm{B}$ & 8.8 & $\mathrm{~F}$ & $\mathrm{P}$ & 1 & $\mathrm{Tr}, \mathrm{Tm}, \mathrm{Ner}$ & 10 & 1112 \\
\hline $\begin{array}{l}\text { Phalacroma acutum (Schütt) } \\
\text { Pavillard }\end{array}$ & $\mathrm{B}$ & 0.6 & M & $\mathrm{P}$ & 1 & Tm, Ner, Oc & 20 & 653 \\
\hline P. argus Stein & $\mathrm{R}$ & 1.5 & M & $\mathrm{P}$ & 1 & $\operatorname{Tr}, \mathrm{Tm}, \mathrm{Ner}, \mathrm{Oc}$ & & \\
\hline P. cuneus Schütt & $\mathrm{R}, \mathrm{B}$ & 4.2 & M & $\mathrm{P}$ & 1 & $\mathrm{Tm}, \mathrm{Tr}, \mathrm{Ner}, \mathrm{Oc}$ & 20 & 558 \\
\hline $\begin{array}{l}\text { P. dolychopterigium Murray et } \\
\text { Whitting }\end{array}$ & $\mathrm{R}$ & 0.3 & M & $\mathrm{P}$ & 1 & Tm, Tr, HS Ner & & \\
\hline P. doryphorum Stein & $\mathrm{R}, \mathrm{B}$ & 20.3 & M & $\mathrm{P}$ & 1 & Tr, Ner, Oc & 20 & 715 \\
\hline P. favus Kofoid et Michener & $\mathrm{R}$ & 3.6 & M & $\mathrm{P}$ & 1 & $\mathrm{Tm}, \mathrm{Tr}, \mathrm{Oc}$ & & \\
\hline P. hindmarchii Murray et Whitting & $\mathrm{R}$ & 0.3 & M & $\mathrm{P}$ & 1 & $\mathrm{Tr}, \mathrm{Oc}$ & & \\
\hline P. mitra Schütt** & $\mathrm{R}, \mathrm{B}$ & 2.7 & M & $\mathrm{P}$ & 1 & $\mathrm{Tm}, \mathrm{Tr}, \mathrm{Ner}, \mathrm{Oc}$ & 10 & 38 \\
\hline P. mucronatum Kofoid et Skogsberg & $\mathrm{B}$ & 0.3 & M & $\mathrm{P}$ & 1 & $\mathrm{Tm}, \mathrm{Oc}$ & 20 & 327 \\
\hline P. operculoides Schütt & $\mathrm{R}, \mathrm{B}$ & 0.6 & $\mathrm{M}$ & $\mathrm{P}$ & 1 & $\mathrm{Tm}, \mathrm{Oc}$ & 30 & 711 \\
\hline P. ovum Schütt & $\mathrm{R}$ & 0.3 & $\mathrm{M}$ & $\mathrm{P}$ & 1 & $\mathrm{Tr}, \mathrm{Oc}$ & & \\
\hline P. porodictyum Stein & $\mathrm{R}, \mathrm{B}$ & 5.5 & $\mathrm{M}$ & $\mathrm{P}$ & 1 & $\mathrm{Tm}, \mathrm{Tr}, \mathrm{Oc}$ & 45 & 120 \\
\hline P. rapa Stein $^{* *}$ & $\mathrm{R}, \mathrm{B}$ & 4.6 & M & $\mathrm{P}$ & 1 & Tm, Tr, Ner, Oc & 10 & 40 \\
\hline $\begin{array}{l}\text { P. rotundata (Claparéde et } \\
\text { Lachmann) Kofoid et Michener** }\end{array}$ & $\mathrm{R}, \mathrm{B}$ & 6.0 & M & $\mathrm{P}$ & 1 & $\mathrm{Tm}, \mathrm{Tr}, \mathrm{Oc}$ & 10 & 721 \\
\hline P. sp. & $\mathrm{B}$ & 3.6 & $\mathrm{M}$ & $\mathrm{P}$ & 1 & $\mathrm{Tr}, \mathrm{Oc}$ & 20 & 273 \\
\hline Podolampas bipes Stein & $\mathrm{R}, \mathrm{B}$ & 16.0 & $\mathrm{~F}$ & $\mathrm{P}$ & 1 & $\mathrm{Tr}, \mathrm{Oc}$ & 13 & 1307 \\
\hline P. palmipes Stein & $\mathrm{R}, \mathrm{B}$ & 7.3 & $\mathrm{~F}$ & $\mathrm{P}$ & 1 & Tr, Sbt, Ner, Oc & 13 & 1124 \\
\hline P. reticulata Kofoid & $\mathrm{R}$ & 1.5 & $\mathrm{~F}$ & $\mathrm{P}$ & 1 & $\mathrm{Tr}, \mathrm{Tm}, \mathrm{Oc}$ & & \\
\hline P. spinifer Okamura & $\mathrm{R}, \mathrm{B}$ & 2.1 & $\mathrm{~F}$ & $\mathrm{P}$ & 1 & $\mathrm{Tr}, \mathrm{Oc}$ & 20 & 163 \\
\hline
\end{tabular}


Apéndice 1. Continuación.

\begin{tabular}{|c|c|c|c|c|c|c|c|c|}
\hline \multirow[b]{2}{*}{ Taxa } & \multirow[b]{2}{*}{ M } & \multirow[b]{2}{*}{ Frec. } & \multirow[b]{2}{*}{$\mathrm{N}$} & \multirow[b]{2}{*}{$\mathrm{H}$} & \multirow[b]{2}{*}{ NO } & \multirow{2}{*}{$\begin{array}{l}\text { Distribución } \\
\text { Global }\end{array}$} & \multicolumn{2}{|c|}{$\begin{array}{l}\text { Abundancia } \\
\text { céls. } \mathrm{L}^{-1}\end{array}$} \\
\hline & & & & & & & Mín. & Máx. \\
\hline Polykrikos kofoidii Chatton & B & 6.0 & $\mathrm{H}$ & $\mathrm{P}$ & 1 & $\mathrm{Tm}, \mathrm{Tr}, \mathrm{Ner}, \mathrm{Oc}$ & 40 & 6533 \\
\hline P. schwartzi Bütschli & B & 10.6 & $\mathrm{H}$ & $\mathrm{P}$ & 1 & $\mathrm{Tr}, \mathrm{Tm}, \mathrm{Ner}$ & 24 & 51630 \\
\hline $\begin{array}{l}\text { Pronoctiluca acuta (Lohmann) J. } \\
\text { Schiller }\end{array}$ & B & 4.2 & $\mathrm{H}$ & $\mathrm{P}$ & 1 & Tm, Tr, Ner & 20 & 1778 \\
\hline P. pelagica Fabre-Domergue & $\mathrm{R}, \mathrm{B}$ & 3.0 & $\mathrm{H}$ & $\mathrm{P}$ & 1 & Tm, Ner & 43 & 2503 \\
\hline P. rostrata F.J.R. Taylor & $\mathrm{R}, \mathrm{B}$ & 7.9 & $\mathrm{H}$ & $\mathrm{P}$ & 1 & Tm, Tr, Ner & 20 & 3428 \\
\hline P. spinifera (Lohmann) Schiller & $\mathrm{B}$ & 0.3 & $\mathrm{H}$ & $\mathrm{P}$ & 1 & Tm, Ner & & 40 \\
\hline $\begin{array}{l}\text { Prorocentrum compressum (Bailey) } \\
\text { Abé ex Dodge }\end{array}$ & $\mathrm{R}, \mathrm{B}$ & 23.3 & $\mathrm{~F}$ & $\mathrm{P}$ & 1 & Tm, Tr, Ner & 10 & 4572 \\
\hline P. gracile Schütt & $\mathrm{R}, \mathrm{B}$ & 73.9 & $\mathrm{~F}$ & $\mathrm{P}$ & 1 & $\mathrm{Tr}, \mathrm{Tm}, \mathrm{Ner}$ & 10 & 12385 \\
\hline $\begin{array}{l}\text { P. lenticulatum (Matzenauer) F.J.R. } \\
\text { Taylor }\end{array}$ & B & 0.6 & $\mathrm{~F}$ & $\mathrm{P}$ & 1 & Tm, Pac, Ner & 20 & 40 \\
\hline P. maximum (Gourret) Schiller & $\mathrm{R}, \mathrm{B}$ & 9.1 & $\mathrm{~F}$ & $\mathrm{P}$ & 1 & Tm, Tr, Ner & 20 & 818 \\
\hline P. micans Ehrenberg* & $\mathrm{R}, \mathrm{B}$ & 61.2 & $\mathrm{~F}$ & $\mathrm{P}$ & 1 & $\mathrm{Tm}, \mathrm{Tr}, \mathrm{Ner}$ & 10 & 15917 \\
\hline $\begin{array}{l}\text { P. minimum (J. Pavillard) J. } \\
\text { Schiller** }\end{array}$ & $\mathrm{R}, \mathrm{B}$ & 9.1 & $\mathrm{~F}$ & $\mathrm{P}$ & 1 & Tm, Tr, HN, Ner & 43 & 5879 \\
\hline P. oblongum (Schiller) F.J.R. Taylor & $\mathrm{R}$ & 1.5 & $\mathrm{~F}$ & $\mathrm{P}$ & 1 & $\mathrm{Tm}, \operatorname{Tr}$ & & \\
\hline $\begin{array}{l}\text { P. rathymum Loeblich, Shirley et R.J. } \\
\text { Schmidt }\end{array}$ & $\mathrm{R}, \mathrm{B}$ & 4.9 & $\mathrm{~F}$ & $\mathrm{~T}$ & 1 & $\mathrm{Tr}, \mathrm{Tm}, \mathrm{Ner}$ & 40 & 716 \\
\hline P. rostratum F. Stein & $\mathrm{R}, \mathrm{B}$ & 11.5 & $\mathrm{~F}$ & $\mathrm{P}$ & 1 & Tr, Sbt, Ner & 10 & 22225 \\
\hline P. sigmoides Böhm & $\mathrm{R}, \mathrm{B}$ & 3.9 & $\mathrm{~F}$ & $\mathrm{P}$ & 1 & $\mathrm{Tm}, \mathrm{Tr}, \mathrm{Ner}$ & 18 & 654 \\
\hline P. triestinum Schiller* & $\mathrm{R}, \mathrm{B}$ & 31.5 & $\mathrm{~F}$ & $\mathrm{P}$ & 1 & Tm, Tr, Ner & 10 & 7355 \\
\hline P. sp. 1 & $\mathrm{~B}$ & 6.9 & $\mathrm{~F}$ & $\mathrm{P}$ & 1 & Tr, Ner & 20 & 3593 \\
\hline P. sp. 2 & B & 0.9 & $\mathrm{~F}$ & $\mathrm{P}$ & 1 & Tr, Ner & 20 & 40 \\
\hline $\begin{array}{l}\text { Protoceratium reticulatum } \\
\text { (Claparède et Lachmann) } \\
\text { Bütschli** }\end{array}$ & $\mathrm{R}, \mathrm{B}$ & 6.9 & $\mathrm{~F}$ & $\mathrm{P}$ & 1 & Tr, Tm, Ner & 10 & 571 \\
\hline $\begin{array}{l}\text { P. spinulosum (Murray et Whitting) } \\
\text { Schiller }\end{array}$ & $\mathrm{R}, \mathrm{B}$ & 0.9 & $\mathrm{~F}$ & $\mathrm{P}$ & 1 & $\mathrm{Tr}, \mathrm{Tm}, \mathrm{Oc}$ & 200 & 1006 \\
\hline $\begin{array}{l}\text { Protoperidinium abei (Paulsen) } \\
\text { Balech }\end{array}$ & $\mathrm{R}, \mathrm{B}$ & 12.4 & $\mathrm{H}$ & $\mathrm{P}$ & 1 & $\mathrm{Tm}, \mathrm{Tr}, \mathrm{Ner}, \mathrm{Oc}$ & 20 & 653 \\
\hline P. bipes (Paulsen) Balech & $\mathrm{R}, \mathrm{B}$ & 8.2 & $\mathrm{H}$ & $\mathrm{P}$ & 1 & Tm, Tr, Ner & 20 & 1423 \\
\hline P. bispinum (Schiller) Balech & $\mathrm{B}$ & 0.3 & $\mathrm{H}$ & $\mathrm{P}$ & 1 & Tm, Ner, Pac & & 100 \\
\hline P. brochii (Kofoid et Swezy) Balech & $\mathrm{R}, \mathrm{B}$ & 16.7 & $\mathrm{H}$ & $\mathrm{P}$ & 1 & Tm, Ner, Oc & 13 & 653 \\
\hline P. capurroi (Balech) Balech & B & 0.3 & $\mathrm{H}$ & $\mathrm{P}$ & 1 & Tr, Tm, Ner & & 89 \\
\hline P. cassum (Balech) Balech var. cassum & $\mathrm{R}, \mathrm{B}$ & 13.9 & $\mathrm{H}$ & $\mathrm{P}$ & 1 & Tm, Ner, Oc & 20 & 3731 \\
\hline $\begin{array}{l}\text { P. cassum var. decens (Balech) } \\
\text { Balech }\end{array}$ & $\mathrm{B}$ & 0.9 & $\mathrm{H}$ & $\mathrm{P}$ & 1 & Tr, Tm, Ner, Oc & 40 & 1067 \\
\hline
\end{tabular}


Apéndice 1. Continuación.

\begin{tabular}{|c|c|c|c|c|c|c|c|c|}
\hline \multirow[b]{2}{*}{ Taxa } & \multirow[b]{2}{*}{ M } & \multirow[b]{2}{*}{ Frec. } & \multirow[b]{2}{*}{$\mathrm{N}$} & \multirow[b]{2}{*}{$\mathrm{H}$} & \multirow[b]{2}{*}{ NO } & \multirow{2}{*}{$\begin{array}{l}\text { Distribución } \\
\text { Global }\end{array}$} & \multicolumn{2}{|c|}{$\begin{array}{c}\text { Abundancia } \\
\text { céls. } \mathrm{L}^{-1}\end{array}$} \\
\hline & & & & & & & Mín. & Máx. \\
\hline P. cerasus (Paulsen) Balech & $\mathrm{R}, \mathrm{B}$ & 9.4 & $\mathrm{H}$ & $\mathrm{P}$ & 1 & Tm, Tr, Ner, Oc & 35 & 2406 \\
\hline P. claudicans (Paulsen) Balech & $\mathrm{B}$ & 8.5 & $\mathrm{H}$ & $\mathrm{P}$ & 1 & $\mathrm{Tr}, \mathrm{Tm}, \mathrm{Ner}$ & 20 & 980 \\
\hline P. compressum (Abé) Balech & $\mathrm{R}, \mathrm{B}$ & 3.3 & $\mathrm{H}$ & $\mathrm{P}$ & 1 & $\mathrm{Tm}, \mathrm{Tr}, \mathrm{Ner}, \mathrm{Oc}$ & 33 & 139 \\
\hline P. conicoides (Paulsen) Balech & $\mathrm{B}$ & 0.6 & $\mathrm{H}$ & $\mathrm{P}$ & 1 & Tm, Ner & 13 & 90 \\
\hline P. conicum (Gran) Balech & $\mathrm{R}, \mathrm{B}$ & 45.8 & $\mathrm{H}$ & $\mathrm{P}$ & 1 & Tm, Tr, Ner & 20 & 4390 \\
\hline $\begin{array}{l}\text { P. corniculum (Kofoid et Michener) } \\
\text { Taylor et Balech }\end{array}$ & B & 4.9 & $\mathrm{H}$ & $\mathrm{P}$ & 1 & $\mathrm{Tr}, \mathrm{Pac}$ & 20 & 4268 \\
\hline P. crassipes (Kofoid) Balech & $\mathrm{R}, \mathrm{B}$ & 4.2 & $\mathrm{H}$ & $\mathrm{P}$ & 1 & $\mathrm{Tm}, \mathrm{Tr}, \mathrm{Ner}$ & 67 & 96 \\
\hline P. cristatum Balech & $\mathrm{B}$ & 0.6 & $\mathrm{H}$ & $\mathrm{P}$ & 1 & Tr, Ner & 20 & 40 \\
\hline $\begin{array}{l}\text { P. denticulatum (Gran et Braarud) } \\
\text { Balech }\end{array}$ & $\mathrm{B}$ & 0.3 & $\mathrm{H}$ & $\mathrm{P}$ & 4 & $\mathrm{Tr}, \mathrm{Tm}, \mathrm{Ner}$ & & 48 \\
\hline P. depressum (Bailey) Balech & $\mathrm{R}, \mathrm{B}$ & 17.3 & $\mathrm{H}$ & $\mathrm{P}$ & 1 & $\mathrm{Tm}, \mathrm{Tr}, \mathrm{Ner}$ & 20 & 4102 \\
\hline P. diabolus (Cleve) Balech & $\mathrm{R}$ & 0.3 & $\mathrm{H}$ & $\mathrm{P}$ & 1 & Tr, Tm, Ner, Oc & & \\
\hline $\begin{array}{l}\text { P. divaricatum (Meunier) Parke et } \\
\text { Dodge }\end{array}$ & B & 11.5 & $\mathrm{H}$ & $\mathrm{P}$ & 1 & Tm, Ner, Oc & 16 & 1825 \\
\hline P. divergens (Ehrenberg) Balech & $\mathrm{R}, \mathrm{B}$ & 51.5 & $\mathrm{H}$ & $\mathrm{P}$ & 1 & Tr, Tm, Ner & 20 & 4564 \\
\hline P. elegans (Cleve) Balech & $\mathrm{R}, \mathrm{B}$ & 7.3 & $\mathrm{H}$ & $\mathrm{P}$ & 1 & $\mathrm{Tr}, \mathrm{Sbt}, \mathrm{Oc}$ & 13 & 394 \\
\hline P. excentricum (Paulsen) Balech & $\mathrm{R}, \mathrm{B}$ & 10.3 & $\mathrm{H}$ & $\mathrm{P}$ & 1 & $\mathrm{Tr}, \mathrm{Tm}, \mathrm{Ner}, \mathrm{Oc}$ & 20 & 520 \\
\hline P. grande (Kofoid) Balech & $\mathrm{R}, \mathrm{B}$ & 36.1 & $\mathrm{H}$ & $\mathrm{P}$ & 1 & $\mathrm{Tr}, \mathrm{Sbt}, \mathrm{Oc}$ & 16 & 9136 \\
\hline P. grani (Ostenfeld) Balech & $\mathrm{R}, \mathrm{B}$ & 21.8 & $\mathrm{H}$ & $\mathrm{P}$ & 1 & $\mathrm{Tm}, \mathrm{Fr}, \mathrm{Ner}, \mathrm{Oc}$ & 20 & 3144 \\
\hline P. hirobis (Abé) Balech & $\mathrm{R}, \mathrm{B}$ & 7.6 & $\mathrm{H}$ & $\mathrm{P}$ & 1 & Tm, Ner, Oc & 13 & 767 \\
\hline P. incognitum (Balech) Balech & B & 0.3 & $\mathrm{H}$ & $\mathrm{P}$ & 1 & Tr, Ner & & 80 \\
\hline P. joergenseni (Balech) Balech & $\mathrm{R}, \mathrm{B}$ & 1.2 & $\mathrm{H}$ & $\mathrm{P}$ & 1 & Tr, Ner, Oc & 40 & 160 \\
\hline P. latidorsale (Dangeard) Balech & $\mathrm{R}, \mathrm{B}$ & 22.7 & $\mathrm{H}$ & $\mathrm{P}$ & 1 & Tm, Ner, Oc & 21 & 2835 \\
\hline P. latispinum (Mangin) Balech & $\mathrm{R}, \mathrm{B}$ & 19.1 & $\mathrm{H}$ & $\mathrm{P}$ & 1 & $\mathrm{Tm}, \mathrm{Tr}, \mathrm{Ner}, \mathrm{Oc}$ & 13 & 5662 \\
\hline P. latissimum (Kofoid) Balech & $\mathrm{R}, \mathrm{B}$ & 9.1 & $\mathrm{H}$ & $\mathrm{P}$ & 1 & $\mathrm{Tr}, \mathrm{Oc}$ & 53 & 567 \\
\hline P. leonis (Pavillard) Balech & $\mathrm{R}, \mathrm{B}$ & 6.1 & $\mathrm{H}$ & $\mathrm{P}$ & 1 & Tm, Tr, Ner, Oc & 28 & 154 \\
\hline P. metananum (Balech) Balech & $\mathrm{B}$ & 0.9 & $\mathrm{H}$ & $\mathrm{P}$ & 1 & $\mathrm{Tm}, \mathrm{Oc}$ & 47 & 454 \\
\hline P. minusculum Pavillard & $\mathrm{R}, \mathrm{B}$ & 8.5 & $\mathrm{H}$ & $\mathrm{P}$ & 1 & Tr, Ner & 43 & 9919 \\
\hline P. mite (Pavillard) Balech & $\mathrm{R}, \mathrm{B}$ & 10.3 & $\mathrm{H}$ & $\mathrm{P}$ & 1 & Tr, Tm, Ner & 20 & 1778 \\
\hline $\begin{array}{l}\text { P. obtusum (Karsten) Parke et } \\
\text { Dodge }\end{array}$ & $\mathrm{R}, \mathrm{B}$ & 4.2 & $\mathrm{H}$ & $\mathrm{P}$ & 1 & $\mathrm{Tm}, \mathrm{Tr}, \mathrm{Ner}$ & 20 & 163 \\
\hline P. oceanicum (Vanhöffen) Balech & $\mathrm{R}, \mathrm{B}$ & 26.4 & $\mathrm{H}$ & $\mathrm{P}$ & 1 & $\mathrm{Tm}, \mathrm{Tr}, \mathrm{Ner}, \mathrm{Oc}$ & 20 & 5005 \\
\hline P. ovatum Pouchet & $\mathrm{R}, \mathrm{B}$ & 6.7 & $\mathrm{H}$ & $\mathrm{P}$ & 1 & Tm, Ner, Oc & 24 & 2754 \\
\hline P. ovum (Paulsen) Balech & $\mathrm{R}, \mathrm{B}$ & 7.9 & $\mathrm{H}$ & $\mathrm{P}$ & 1 & Tm, Ner, Oc & 20 & 871 \\
\hline P. parcum (Balech) Balech & $\mathrm{B}$ & 0.6 & $\mathrm{H}$ & $\mathrm{P}$ & 1 & Tm, Ner, Oc & 81 & 280 \\
\hline P. cf. parvicollum (Balech) Balech & $\mathrm{B}$ & 1.5 & $\mathrm{H}$ & $\mathrm{P}$ & 1 & Fr, Tm, Ner & 58 & 4497 \\
\hline P. parviventer (Gaarder) Balech & $\mathrm{B}$ & 14.6 & $\mathrm{H}$ & $\mathrm{P}$ & 1 & $\mathrm{Tm}, \mathrm{Tr}, \mathrm{Ner}$ & 24 & 2564 \\
\hline
\end{tabular}


Apéndice 1. Continuación.

\begin{tabular}{|c|c|c|c|c|c|c|c|c|}
\hline \multirow[b]{2}{*}{ Taxa } & \multirow[b]{2}{*}{ M } & \multirow[b]{2}{*}{ Frec. } & \multirow[b]{2}{*}{$\mathrm{N}$} & \multirow[b]{2}{*}{$\mathrm{H}$} & \multirow[b]{2}{*}{$\mathrm{NO}$} & \multirow{2}{*}{$\begin{array}{l}\text { Distribución } \\
\text { Global }\end{array}$} & \multicolumn{2}{|c|}{$\begin{array}{l}\text { Abundancia } \\
\text { céls. } L^{-1}\end{array}$} \\
\hline & & & & & & & Mín. & Máx. \\
\hline P. parvispinum Balech & $\mathrm{R}$ & 0.6 & $\mathrm{H}$ & $\mathrm{P}$ & 1 & Tr, Tm, Ner & & \\
\hline P. pellucidum Bergh & $\mathrm{R}$ & 0.3 & $\mathrm{H}$ & $\mathrm{P}$ & 1 & $\mathrm{Tm}, \mathrm{Tr}, \mathrm{Ner}, \mathrm{Oc}$ & & \\
\hline P. pentagonum (Gran) Balech & $\mathrm{R}, \mathrm{B}$ & 23.3 & $\mathrm{H}$ & $\mathrm{P}$ & 1 & $\mathrm{Tm}, \mathrm{Tr}, \mathrm{Ner}, \mathrm{Oc}$ & 20 & 789 \\
\hline P. punctulatum (Gran) Balech & $\mathrm{R}, \mathrm{B}$ & 12.7 & $\mathrm{H}$ & $\mathrm{P}$ & 1 & $\mathrm{Tm}, \mathrm{Tr}, \mathrm{Ner}, \mathrm{Oc}$ & 20 & 1307 \\
\hline P. pyriforme (Paulsen) Balech & $\mathrm{R}, \mathrm{B}$ & 2.1 & $\mathrm{H}$ & $\mathrm{P}$ & 1 & Tm, Ner, Oc & 40 & 474 \\
\hline P. pyrum (Balech) Balech & $\mathrm{R}, \mathrm{B}$ & 26.4 & $\mathrm{H}$ & $\mathrm{P}$ & 1 & Tr, Tm, Ner & 18 & 7660 \\
\hline P. quarnerense (Schröder) Balech & $\mathrm{R}, \mathrm{B}$ & 21.5 & $\mathrm{H}$ & $\mathrm{P}$ & 1 & Tr, Sbt, Ner, Oc & 13 & 192 \\
\hline $\begin{array}{l}\text { P. robustum (Meunier) Hernández- } \\
\text { Becerril }\end{array}$ & $\mathrm{R}$ & 0.3 & $\mathrm{H}$ & $\mathrm{P}$ & 1 & Tr, Pac. & & \\
\hline P. roseum (Paulsen) Balech & $\mathrm{R}$ & 1.5 & $\mathrm{H}$ & $\mathrm{P}$ & 1 & Tm, Ner, Oc & & \\
\hline P. solidicorne (Mangin) Balech & B & 0.3 & $\mathrm{H}$ & $\mathrm{P}$ & 1 & Tr, Sbt, Ner, Oc & & 30 \\
\hline P. steinii (Jörgensen) Balech & B & 1.5 & $\mathrm{H}$ & $\mathrm{P}$ & 1 & Tm, Tr, Ner, Oc & 20 & 2845 \\
\hline P. subpyriforme (Dangeard) Balech & $\mathrm{R}, \mathrm{B}$ & 2.4 & $\mathrm{H}$ & $\mathrm{P}$ & 1 & Tm, Ner, Oc & 29 & 3428 \\
\hline P. subsphaericum (Balech) Balech & $\mathrm{R}, \mathrm{B}$ & 5.5 & $\mathrm{H}$ & $\mathrm{P}$ & 1 & Tm, Ner, Oc & 27 & 4497 \\
\hline P. tenuissimum (Kofoid) Balech & $\mathrm{R}, \mathrm{B}$ & 0.9 & $\mathrm{H}$ & $\mathrm{P}$ & 1 & $\mathrm{Tr}, \mathrm{Tm}, \mathrm{Ner}, \mathrm{Oc}$ & 50 & 22195 \\
\hline P. thorianum (Paulsen) Balech & $\mathrm{R}, \mathrm{B}$ & 1.5 & $\mathrm{H}$ & $\mathrm{P}$ & 1 & $\operatorname{Tm}, \operatorname{Tr}$ & 50 & 76 \\
\hline P. tristylum (Stein) Balech & $\mathrm{R}$ & 0.3 & $\mathrm{H}$ & $\mathrm{P}$ & 1 & Tm, Ner, Oc & & \\
\hline P. tuba (Schiller) Balech & $\mathrm{R}, \mathrm{B}$ & 3.0 & $\mathrm{H}$ & $\mathrm{P}$ & 1 & Tm, Ner, Oc & 14 & 160 \\
\hline P. venustum (Matzenauer) Balech & $\mathrm{R}, \mathrm{B}$ & 10.6 & $\mathrm{H}$ & $\mathrm{P}$ & 1 & $\mathrm{Tm}, \mathrm{Tr}, \mathrm{Oc}$ & 20 & 2453 \\
\hline P. vulgare Balech & B & 0.6 & $\mathrm{H}$ & $\mathrm{P}$ & 1 & Tm, Ner, Oc & 13 & 35 \\
\hline P. sp. 1 & $\mathrm{~B}$ & 25.5 & $\mathrm{H}$ & $\mathrm{P}$ & 1 & Tr, Ner & 10 & 4960 \\
\hline P. sp. 2 & $\mathrm{~B}$ & 17.9 & $\mathrm{H}$ & $\mathrm{P}$ & 1 & Tr, Ner & 10 & 3920 \\
\hline $\begin{array}{l}\text { Ptychodiscus noctiluca Murray ex } \\
\text { Haeckel }\end{array}$ & $\mathrm{R}$ & 0.6 & M & $\mathrm{P}$ & 1 & $\mathrm{Tm}, \mathrm{Tr}, \mathrm{Ner}, \mathrm{Oc}$ & 20 & 120 \\
\hline $\begin{array}{l}\text { Pyrocystis fusiformis Wyville- } \\
\text { Thompson ex Blackmann }\end{array}$ & $\mathrm{R}$ & 0.9 & $\mathrm{~F}$ & $\mathrm{P}$ & 2 & $\mathrm{Tr}, \mathrm{Oc}$ & & \\
\hline P. gerbaulti Pavillad & $\mathrm{R}$ & 1.5 & $\mathrm{~F}$ & $\mathrm{P}$ & 2 & $\mathrm{Tr}, \mathrm{Oc}$ & & \\
\hline P. lunula (Schütt) Schütt & $\mathrm{R}, \mathrm{B}$ & 1.2 & $\mathrm{~F}$ & $\mathrm{P}$ & 2 & $\mathrm{Tr}, \mathrm{Oc}$ & 10 & 20 \\
\hline P. noctiluca Stein & $\mathrm{R}, \mathrm{B}$ & 8.2 & $\mathrm{~F}$ & $\mathrm{P}$ & 2 & $\mathrm{Tr}, \mathrm{Oc}$ & 20 & 3688 \\
\hline P. robusta Kofoid & $\mathrm{R}, \mathrm{B}$ & 11.2 & $\mathrm{~F}$ & $\mathrm{P}$ & 2 & $\mathrm{Tr}, \mathrm{Oc}$ & 33 & 2020 \\
\hline $\begin{array}{l}\text { Pyrodinium bahamense var. } \\
\text { compressum (Böhm) Steidinger, } \\
\text { Tester et Taylor** }\end{array}$ & $\mathrm{R}, \mathrm{B}$ & 34.8 & $\mathrm{~F}$ & $\mathrm{P}$ & 4 & Tr, Ner, Oc & 20 & 773554 \\
\hline Pyrophacus horologium Stein & $\mathrm{R}, \mathrm{B}$ & 14.2 & $\mathrm{~F}$ & $\mathrm{P}$ & 1 & Tr, Tm, Ner, Oc & 20 & 2453 \\
\hline P. steinii (Schiller) Wall et Dale & $\mathrm{R}, \mathrm{B}$ & 26.9 & $\mathrm{~F}$ & $\mathrm{P}$ & 1 & $\mathrm{Tr}, \mathrm{Tm}, \mathrm{Ner}, \mathrm{Oc}$ & 20 & 1307 \\
\hline $\begin{array}{l}\text { P. vancampoae (M. Rossignol) Wall } \\
\text { et Dale }\end{array}$ & $\mathrm{R}$ & 1.2 & $\mathrm{~F}$ & $\mathrm{P}$ & 1 & $\mathrm{Tm}, \mathrm{Tr}, \mathrm{Ner}, \mathrm{Oc}$ & & \\
\hline Scaphodinium miriabile Margalef & $\mathrm{R}, \mathrm{B}$ & 1.2 & $\mathrm{H}$ & $\mathrm{P}$ & 1 & $\mathrm{Tr}, \mathrm{Tm}, \mathrm{Oc}$ & 37 & 65 \\
\hline
\end{tabular}


Apéndice 1. Continuación.

\begin{tabular}{|c|c|c|c|c|c|c|c|c|}
\hline \multirow[b]{2}{*}{ Taxa } & \multirow[b]{2}{*}{ M } & \multirow[b]{2}{*}{ Frec. } & \multirow[b]{2}{*}{$\mathrm{N}$} & \multirow[b]{2}{*}{$\mathrm{H}$} & \multirow[b]{2}{*}{$\mathrm{NO}$} & \multirow{2}{*}{$\begin{array}{l}\text { Distribución } \\
\text { Global }\end{array}$} & \multicolumn{2}{|c|}{$\begin{array}{l}\text { Abundancia } \\
\text { céls. } L^{-1}\end{array}$} \\
\hline & & & & & & & Mín. & Máx. \\
\hline Schuettiella mitra (Schütt) Balech & $\mathrm{R}$ & 0.9 & $\mathrm{~F}$ & $\mathrm{P}$ & 1 & $\mathrm{Tr}, \mathrm{Oc}$ & & \\
\hline $\begin{array}{l}\text { Scrippsiella trochoidea (Stein) } \\
\text { Steidinger et Balech* }\end{array}$ & $\mathrm{R}, \mathrm{B}$ & 23.3 & $\mathrm{~F}$ & $\mathrm{P}$ & 1 & $\mathrm{Tr}, \mathrm{Tm}, \mathrm{Ner}$ & 25 & 111123 \\
\hline S. sweeneyae Balech & $\mathrm{B}$ & 21.2 & $\mathrm{~F}$ & $\mathrm{P}$ & 1 & Tm, Ner & 39 & 27127 \\
\hline S. sp. 1 & $\mathrm{~B}$ & 40.0 & $\mathrm{~F}$ & $\mathrm{P}$ & 1 & Tr, Ner & 40 & 268110 \\
\hline S. sp. 2 & $\mathrm{~B}$ & 15.2 & $\mathrm{~F}$ & $\mathrm{P}$ & 1 & Tr, Ner & 20 & 66863 \\
\hline $\begin{array}{l}\text { Spatulodinium pseudonoctiluca } \\
\text { (Pouchet) J. Cachon et M. Cachon } \\
\text { ex Loeblich Jr. et Loeblich III }\end{array}$ & $\mathrm{B}, \mathrm{V}$ & 0.6 & $\mathrm{H}$ & $\mathrm{P}$ & 1 & $\mathrm{Tr}, \mathrm{Tm}, \mathrm{Oc}$ & 77 & 78 \\
\hline $\begin{array}{l}\text { Spiraulax jollifei (Murray et } \\
\text { Whitting) Kofoid }\end{array}$ & $\mathrm{R}$ & 0.9 & $\mathrm{~F}$ & $\mathrm{P}$ & 1 & $\mathrm{Tr}, \mathrm{Oc}$ & & \\
\hline S. kofoidii Graham & $\mathrm{R}$ & 1.5 & $\mathrm{~F}$ & $\mathrm{P}$ & 1 & Tr, Sbt, Ner, Oc & & \\
\hline $\begin{array}{c}\text { Takayama cf. cladochroma (Larsen) } \\
\text { de Salas, Bolch et Hallegraeff** }\end{array}$ & $\mathrm{V}$ & 0.3 & $\mathrm{~F}$ & & 1 & Sbt, Ner & & \\
\hline $\begin{array}{l}\text { Torodinium teredo (Pouchet) Kofoid } \\
\text { et Swezy }\end{array}$ & B & 5.5 & $\mathrm{~F}$ & $\mathrm{P}$ & 1 & Tm, Tr, Ner, Oc & 38 & 3201 \\
\hline T. robustum Kofoid et Swezy & $\mathrm{B}$ & 38.5 & $\mathrm{~F}$ & $\mathrm{P}$ & 1 & Tr, Tm, Ner & 20 & 8051 \\
\hline Warnowia juno (Schütt) Schiller & $\mathrm{B}$ & 18.8 & $\mathrm{H}$ & $\mathrm{P}$ & 1 & $\mathrm{Tr}, \mathrm{Tm}, \mathrm{Ner}$ & 13 & 2369 \\
\hline $\begin{array}{l}\text { W. maxima (Kofoid et Swezy) } \\
\text { Lindemann }\end{array}$ & B & 6.9 & $\mathrm{H}$ & $\mathrm{P}$ & 1 & $\mathrm{Tr}, \mathrm{Tm}, \mathrm{Ner}$ & 20 & 608 \\
\hline W. polyphemus (Pouchet) Schiller & $\mathrm{B}$ & 16.4 & $\mathrm{H}$ & $\mathrm{P}$ & 1 & $\mathrm{Tr}, \mathrm{Tm}, \mathrm{Ner}, \mathrm{Oc}$ & 16 & 5556 \\
\hline W. rosea (Pouchet) Schiller & $\mathrm{B}$ & 7.6 & $\mathrm{H}$ & $\mathrm{P}$ & 1 & $\mathrm{Tr}, \mathrm{Tm}, \mathrm{Ner}$ & 20 & 2503 \\
\hline $\begin{array}{l}\text { W. violecens (Kofoid et Swezy) } \\
\text { Lindemann }\end{array}$ & $\mathrm{V}$ & 0.9 & $\mathrm{H}$ & $\mathrm{P}$ & 1 & Tm, Ner, Oc & 22 & 40 \\
\hline \multicolumn{9}{|l|}{ División Heterocontophyta } \\
\hline $\begin{array}{l}\text { Chattonella marina var. antiqua } \\
\text { (Hada) Demura et Kawachi** }\end{array}$ & $\mathrm{B}, \mathrm{V}$ & 1.8 & $\mathrm{~F}$ & $\mathrm{P}$ & 1 & Tm, Ner, Oc & 48 & 87 \\
\hline $\begin{array}{l}\text { Dictyocha fibula Ehrenberg var. } \\
\text { fibula }\end{array}$ & $\mathrm{R}, \mathrm{B}$ & 24.9 & $\mathrm{~F}$ & $\mathrm{P}$ & 1 & $\mathrm{Tm}, \mathrm{Oc}$ & 10 & 4195 \\
\hline $\begin{array}{l}\text { D. fibula var. robusta Schrader et } \\
\text { Murray }\end{array}$ & B & 1.8 & $\mathrm{~F}$ & $\mathrm{P}$ & 1 & Tm, Ner & 24 & 108 \\
\hline $\begin{array}{l}\text { Octactis octonaria (Ehrenberg) } \\
\text { Hovasse }\end{array}$ & B & 6.9 & $\mathrm{~F}$ & $\mathrm{P}$ & 1 & $\mathrm{Tm}, \mathrm{Oc}$ & 27 & 4482 \\
\hline $\begin{array}{l}\text { Fibrocapsa japonica Toriumi et } \\
\text { Takano** }\end{array}$ & $\mathrm{V}$ & 0.6 & $\mathrm{~F}$ & $\mathrm{P}$ & 1 & $\mathrm{Tm}, \mathrm{Ner}, \mathrm{Oc}$ & & \\
\hline cf. Hillea sp. & B & 0.3 & $\mathrm{~F}$ & $\mathrm{P}$ & 1 & $\mathrm{Tm}, \mathrm{Tr}, \mathrm{Ner}$ & 283 & 35626 \\
\hline
\end{tabular}


Apéndice 1. Continuación.

\begin{tabular}{|c|c|c|c|c|c|c|c|c|}
\hline \multirow[b]{2}{*}{ Таха } & \multirow[b]{2}{*}{ M } & \multirow[b]{2}{*}{ Frec. } & \multirow[b]{2}{*}{$\mathrm{N}$} & \multirow[b]{2}{*}{$\mathrm{H}$} & \multirow[b]{2}{*}{ NO } & \multirow{2}{*}{$\begin{array}{l}\text { Distribución } \\
\text { Global }\end{array}$} & \multicolumn{2}{|c|}{$\begin{array}{l}\text { Abundancia } \\
\text { céls. } \mathrm{L}^{-1}\end{array}$} \\
\hline & & & & & & & Mín. & Máx. \\
\hline \multicolumn{9}{|l|}{ División Prymnesiophyta } \\
\hline Calciosolenia murrayi Gran & $\mathrm{R}$ & 0.6 & $\mathrm{~F}$ & $\mathrm{P}$ & 1 & $\mathrm{Tm}, \mathrm{Oc}$ & & \\
\hline Coccolithus sp. & B & 1.5 & $\mathrm{~F}$ & $\mathrm{P}$ & 1 & $\mathrm{Tm}, \mathrm{Oc}$ & 89 & 3972 \\
\hline Phaeocystis sp.* & B & 6.9 & $\mathrm{~F}$ & $\mathrm{P}$ & 3 & Tm, Ner, Oc & 55 & 202899 \\
\hline $\begin{array}{l}\text { Umbilicosphaera cf. sibogae (Weber- } \\
\text { van Bosse) Gaarder }\end{array}$ & $\mathrm{B}$ & 0.3 & $\mathrm{~F}$ & $\mathrm{P}$ & 1 & $\mathrm{Tm}, \mathrm{Tr}, \mathrm{Oc}$ & 20 & 667 \\
\hline \multicolumn{9}{|l|}{ División Chlorophyta } \\
\hline $\begin{array}{l}\text { Pediastrum clathratum (Schroder) } \\
\text { Lemmermann }\end{array}$ & $\mathrm{B}$ & 0.3 & $\mathrm{~F}$ & $\mathrm{P}$ & 3 & Dul. & & 40 \\
\hline P. duplex Meyen & $\mathrm{B}$ & 0.3 & $\mathrm{~F}$ & $\mathrm{P}$ & 3 & Dul. & & 80 \\
\hline \multicolumn{9}{|l|}{ División Euglenophyta } \\
\hline Euglena pascheri Swirenko & $\mathrm{B}, \mathrm{V}$ & 47.3 & $\mathrm{~F}$ & $\mathrm{P}$ & 1 & Ner & 10 & 72418 \\
\hline Eutreptia marina da Cunha & $\mathrm{B}$ & 12.4 & $\mathrm{~F}$ & $\mathrm{P}$ & 1 & Ner & 58 & 528 \\
\hline \multicolumn{9}{|l|}{ División Cyanophyta } \\
\hline Rhichelia intracelullaris Schmidt & $\mathrm{R}, \mathrm{B}$ & 0.3 & $\mathrm{~F}$ & $\mathrm{P}$ & 4 & Ner & & 856 \\
\hline Synechococcus sp. & $\mathrm{B}, \mathrm{V}$ & 0.6 & $\mathrm{~F}$ & $\mathrm{P}$ & 2 & Ner & 10 & 698 \\
\hline Synechocystis sp. & $\mathrm{B}, \mathrm{V}$ & 0.3 & $\mathrm{~F}$ & $\mathrm{P}$ & 2 & Ner & & 715 \\
\hline $\begin{array}{l}\text { Trichodesmium erythraeum } \\
\text { Ehrenberg** }\end{array}$ & $\mathrm{R}, \mathrm{B}$ & 1.2 & $\mathrm{~F}$ & $\mathrm{P}$ & 4 & $\mathrm{Tr}, \mathrm{Tm}, \mathrm{Oc}$ & 20 & 212541 \\
\hline $\begin{array}{l}\text { T. sp. } \\
\text { Phylum Zoomastigophora }\end{array}$ & $\mathrm{R}, \mathrm{B}$ & 0.3 & $\mathrm{~F}$ & $\mathrm{P}$ & 2 & Tr, Ner & 20 & 597 \\
\hline Choanoflagelado & $\mathrm{B}$ & 3.9 & $\mathrm{H}$ & $\mathrm{P}$ & 1 & $\mathrm{Tr}, \mathrm{Ner}, \mathrm{Oc}$ & 20 & 280 \\
\hline
\end{tabular}




\section{APÉNDICE 2}

Esquema de clasificación de los taxa por división algal, hasta nivel de género; entre paréntesis se especifica el número de taxa de cada género registrados en la Bahía de Acapulco.

\section{División Bacillariophyta}

Clase Coscinodiscophyceae Round et Crawford

Subclase Thalassiosirophycidae Round et

Crawford

Orden Thalassiosirales Glezer et Makarova

Familia Thalassiosiraceae Lebour

Planktoniella Schütt (2)

Thalassiosira Cleve emend. Hasle (11)

Familia Skeletonemataceae Lebour

Detonula Schütt ex De Tony (1)

Skeletonema Greville (4)

Familia Stephanodiscaceae Glezer et

Makarova

Cyclotella Kützing (2)

Familia Lauderiaceae (Schütt) Lemmermann Lauderia Cleve (1)

Subclase Coscinodiscophycidae Round et

Crawford

Orden Melosirales Crawford

Familia Melosiraceae Kützing Melosira Agardh (1)

Familia Stephanopyxidae Nikolaev Stephanopyxis (Ehrenberg) Ehrenberg (2)

Orden Paraliales Crawford

Familia Paraliaceae Crawford

Paralia Heiberg (1)

Orden Aulacoseirales Crawford

Familia Aulacoceiraceae Crawford Aulacoseira Thwaites (1)

Orden Coscinodiscales Round et Crawford

Familia Coscinodiscaceae Kützing Coscinodiscus Ehrenberg (8)

Stellarima Hasle et Sims (1)

Familia Gossleriellaceae Round Gossleriella Schütt (1)

Familia Hemidiscaceae Hendey emend. Simonsen

Actinocyclus Ehrenberg (4)

Azpeitia (Schmidt) Fryxell et Sims (1)

Hemidiscus Wallich (3)

Roperia Grunow ex Pelletan (1)
Familia Heliopeltaceae Smith

Actinoptychus Ehrenberg (6)

Orden Asterolamprales Round et Crawford

Familia Asterolampraceae Smith

Asteromphalus Ehrenberg (6)

Spatangidium Brébisson (1)

Subclase Biddulphiophycidae Round et

Crawford

Orden Triceratiales Round et Crawford

Familia Triceratiaceae (Schütt) Lemmermann

Cerataulus Ehrenberg (1)

Eupodiscus Bailey (1)

Odontella C.A. Agardh (4)

Triceratium Ehrenberg (2)

Familia Plagiogrammaceae De Toni

Plagiogramma Greville (1)

Orden Biddulphiales Krieger

Familia Biddulphiaceae Kützing

Biddulphia Gray (3)

Isthmia Agardh (1)

Orden Hemiaulales Round et Crawford

Familia Hemiaulaceae Heiberg

Cerataulina H. Peragallo ex Schütt (1)

Climacodium Grunow (1)

Eucampia Ehrenberg (2)

Hemiaulus Heiberg (3)

Familia Bellerocheaceae Crawford

Bellerochea van Heurck emend. von Stosch

(2)

Familia Streptothecaceae Crawford

Helicotheca Richard (1)

Subclase Lithodesmiophycidae Round et

Crawford

Orden Lithodesmiales Round et Crawford

Familia Lithodesmiaceae Round

Ditylum Bailey (1)

Lithodesmium Ehrenberg (1)

Subclase Corethrophycidae Round et Crawford

Orden Corethrales Round et Crawford

Familia Corethraceae Lebour

Corethron Castracane (1) 
Apéndice 2. Continuación.

\begin{tabular}{|c|c|}
\hline Subclase Cymatosirophycidae Round et & Thalassionema (Grunow) Hustedt (6) \\
\hline Crawford & Thalassiothrix Cleve et Grunow (1) \\
\hline Orden Cymatosirales Round et Crawford & Orden Striatellales Round \\
\hline Familia Cymatosiraceae Hasle, von Stosch et & Familia Striatellaceae Kützing \\
\hline Syvertsen & Grammatophora Ehrenberg (2) \\
\hline Campylosira Grunow ex van Heueck (1) & Orden Climacospheniales Round \\
\hline Cymatosira Grunow (1) & Familia Climacosphemaceae Round \\
\hline Subclase Rhizosoleniophycidae Round et & Climacosphenia Ehrenberg (1) \\
\hline Crawford & Clase Bacillariophyceae Round, Crawford et \\
\hline Orden Rhizosoleniales Silva & Mann \\
\hline Familia Rhizosoleniaceae De Toni & Orden Lyrellales D.G. Mann \\
\hline Dactyliosolen Castracane (2) & Familia Lyrellaceae D.G. Mann \\
\hline Guinardia H. Peragallo (4) & Lyrella Karajeva (4) \\
\hline Neocalyptrella Hernández-Becerril et & Petroneis Stickle et D.G. Mann (1) \\
\hline Meave del Castillo (1) & Orden Mastogloiales D.G. Mann \\
\hline Proboscia Sundström (4) & Familia Mastogloiaceae Mereschkowsky \\
\hline Pseudoguinardia von Stosch (1) & Mastogloia Thwaites (2) \\
\hline Pseudosolenia Sundström (1) & Orden Achnanthales Silva \\
\hline Rhizosolenia Brightwell (13) & Familia Achnanthaceae Kützing \\
\hline Subclase Chaetocerotophycidade Round et & Achnanthes Bory (3) \\
\hline Crawford & Familia Cocconeidaceae Kützing \\
\hline Orden Chaetocerotales Round et Crawford & Cocconeis Ehrenberg (6) \\
\hline Familia Chaetocerotaceae Ralf in Pritchard & Orden Naviculales Bessey \\
\hline Bacteriastrum Shadbolt (5) & Suborden Sellaphorineae D.G. Mann \\
\hline Chaetoceros Ehrenberg (48) & Familia Pinnulariaceae D.G. Mann \\
\hline Orden Leptocylindrales Round et Crawford & Pinnularia Ehrenberg (1) \\
\hline Familia Leptocylindraceae Lebour & Suborden Diploneidineae Mann \\
\hline Leptocylindrus Cleve (3) & Familia Diploneidaceae Mann \\
\hline Clase Fragilariophyceae Round, Crawford et & Diploneis Ehrenberg (7) \\
\hline Mann & Suborden Naviculineae Hendey \\
\hline Subclase Fragilariophycidae Round & Familia Naviculaceae Kützing \\
\hline Orden Fragilariales Silva & Haslea Simonsen (4) \\
\hline Familia Fragilariaceae Greville & Navicula Bory (4) \\
\hline Asterionellopsis Round (1) & Trachyneis Cleve (1) \\
\hline Fragilaria Lyngbye (1) & Familia Pleurosigmataceae Mereschkowsky \\
\hline Podocystis Bailey (1) & Gyrosigma Hassall (2) \\
\hline Synedra Ehrenberg (2) & Pleurosigma Wm. Smith (7) \\
\hline Orden Licmophorales Round & Familia Plagiotropidaceae Mann \\
\hline Familia Licmophoracae Kützing & Plagiotropis Cleve (1) \\
\hline Licmophora Agardh (4) & Familia Stauroneidaceae Mann \\
\hline Orden Rhaphoneidales Round & Meuniera P.C. Silva (1) \\
\hline Familia Rhaphoneidaceae Forti & Orden Thalassiophysales D.G. Mann \\
\hline Delphineis Andrews (2) & Familia Catenulaceae Mereschkowsky \\
\hline Orden Thalassionematales Round & Amphora Ehrenberg (6) \\
\hline Familia Thalassionemataceae Round & Orden Bacillariales Hendey \\
\hline Lioloma Hasle (2) & Familia Bacillariaceae Ehrenberg \\
\hline
\end{tabular}


Apéndice 2. Continuación.

Alveus Kaczmarska et Fryxell (1)
Bacillaria Gmelin (1)
Cylindrotheca Rabenhorst (1)
Cymatonitzschia Simonsen (1)
Fragilariopsis Hustedt (1)
Hantzschia Grunow (1)
Nitzschia Hassall (10)
Psammodictyon D.G. Mann (2)

Pseudo-nitzschia H. Peragallo et Peragallo

(7)

Tryblionella W. Smith (1)

Orden Rhopalodiales Mann

Familia Rhopalodiaceae (Karsten)

Topachevskyj et Oksiyuk

Rhopalodia Müller (1)

Orden Surirellales D.G. Mann

Familia Entomoneidaceae Reimer

Entomoneis Ehrenberg (2)

Familia Surirellaceae Kützing

Campylodiscus Ehrenberg (1)

Surirella Turpin (3)

\section{División Dinophyta}

Subdivisión Dinokaryota Fensome et al.

Clase Dinophyceae Pascher

Subclase Gymnodiniphycidae Fensome et al.

Orden Gymnodiniales Apstein

Suborden Gymnodiniineae Apstein

Familia Gymnodiniaceae (Bergh) Lankester Akashiwo G. Hansen et O. Moestrup (1)

Amphidinium Claparéde et Lachmann (4)

Cochlodinium Schütt (4)

Gymnodinium Stein (18)

Gyrodinium Kofoid et Swezy (10)

Karenia G. Hansen et O. Moestrup (8)

Katodinium Fott (1)

Takayama de Salas, Bolch et Hallegraeff

(1)

Torodinium Kofoid et Swezy (2)

Familia Polykrikaceae Kofoid et Swezy

Polykrikos Bütschli (2)

Familia Warnowiaceae Lindemann

Ceratoperidinium Margalef ex Loeblich III (1)

Erythropsidinium P.C. Silva (1)

Greuetodinium Loeblich III (1)
Nematodinium Kofoid et Swezy (1)

Warnowia Lindemann (5)

Familia incierta

Gynogonadinium Gómez (1)

Orden Ptychodiscales Fensome et al.

Familia Ptychodiscaceae Willey et Hickson

Subfamilia Ptychodiscoideae Willey et

Hickson

Balechina Loeblich et Loeblich (1)

Ptychodiscus Stein (1)

Familia Amphitholaceae Poche

Achradina Lohmann (1)

Familia Brachydiniaceae Sournia

Brachydinum F.J.R. Taylor (1)

Asterodinium Sournia (1)

Subclase Peridiniphycidae Fensome et al.

Orden Gonyaulacales Taylor

Suborden Gonyaulacineae

Familia Gonyaulacaceae Lindemann

Subfamilia Cribroperidinioideae Fensome et al.

Lingulodinium Wall (1)

Protoceratium Bergh (2)

Subfamilia Gonyaulacoideae Lindemann

Gonyaulax Diesing (16)

Schuettiella Balech (1)

Spiraulax Kofoid (2)

Familia Ceratocoryaceae Lindemann

Ceratocorys Stein (4)

Suborden Ceratiineae Fensome et al.

Familia Ceratiaceae Willey et Hickson

Neoceratium Gómez, Moreira et LópezGarcía (69)

Suborden Goniodomineae Fensome et al.

Familia Goniodomaceae Lindemann

Subfamilia Goniodomoideae Lindemann

Goniodoma Stein (2)

Subfamilia Helgolandinioideae Fensome et al.

Alexandrium Halim (5)

Fragilidium Balech ex Loeblich (2)

Pyrophacus Stein (3)

Subfamilia Pyrodinioideae Fensome et al.

Pyrodinium Plate (1)

Familia Pyrocystaceae Apstein

Pyrocystis Murray ex Haeckel (5) 
Apéndice 2. Continuación.
Suborden incierto (Gonyaulacales) Fensome et al.
Familia Heterodiniaceae Lindemann Heterodinium Kofoid (2)
Familia incierta (Gonyaulacales, Suborden incierto) Fensome et al.
Peridiniella Kofoid et Michener (1)
Orden Peridiniales Haeckel
Suborden Heterocapsineae Fensome et al.
Familia Heterocapsaceae Fensome et al.
Heterocapsa Stein (1)
Suborden Peridiniineae Haeckel
Familia Peridiniaceae Ehrenberg
Subfamilia Calciodinelloideae Fensome et al.
Ensiculifera Balech ex Cox et Arnott (2)
Scrippsiella Balech ex Loeblich III (4)
Subfamilia Peridinioideae Ehrenberg
Peridinium Ehrenberg (1)
Familia Congruentidiaceae Schiller
Subfamilia Congruentidioideae Fensome et al.
Protoperidinium Bergh (62)
Subfamilia Diplopsalioideae
Diplopsalis Bergh (1)
Diplopsalopsis Meunier (2)
Familia Podolampaceae Lindemann
Blepharocysta Ehrenberg (1)
Podolampas Stein (4)
Orden incierto (Suborden Peridinnineae)
Fensome et al.
Familia Oxytoxaceae Lindemann

Corythodinium Loeblich Jr. et Loeblich III

Oxytoxum Stein (10)

Subclase Dinophysiphycidae Möhn ex

Fensome et al.

Orden Dinophysiales Kofoid

Familia Amphisoleniaceae Lindemann Amphisolenia Stein (2)

Familia Oxyphysiaceae Sournia

Oxyphysis Kofoid (1)

Familia Dinophysiaceae Stein

Dinophysis Ehrenberg (16)

Histioneis Stein (2)

Metaphalacroma Tai et Skogsberg (1)
Ornithocercus Stein (8)

Phalacroma Stein (15)

Subclase Prorocentrophycidae Fensome et al.

Orden Prorocentrales Lemmermann

Familia Prorocentraceae Stein

Prorocentrum Ehrenberg (13)

Clase Blastodiniphyceae Fensome et al.

Orden Blastodiniales Chatton

Familia Oodiniaceae Chatton

Oodinium Chatton (1)

Familia Cachonellaceae Silva

Dissodinium Klebs in Pascher (1)

Familia incierta

Chytriodinium Chatton (1)

Clase Noctiluciphyceae Fensome et al.

Orden Noctilucales Haeckel

Familia Kofoidiniaceae F.J.R Taylor

Kofoidinium Pavillard (3)

Spatulodinium J. Cachon et M. Cachon (1)

Familia Leptodiscaceae Taylor

Scaphodinium Kofoid (1)

Familia Noctilucaceae Saville-Kent

Noctiluca Suriray (1)

Pronoctiluca Fabre-Domergue (4)

\section{División Heterocontophyta}

Clase Dictyochophyceae Silva

Orden Dictyochales Haeckel

Familia Dictyochaceae Lemmermann

Dictyocha Ehrenberg (2)

Octactis Schiller (1)

Clase Raphidophyceae Chadefaud

Orden Chattonellales Throndsen

Familia Chattonellaceae Throndsen

Chattonella Biecheler (1)

Fibrocapsa Toriumi et Takano (1)

Clase Cryptophyceae Fritsch

Orden Chryptomonadales Engler

Familia Hillaceae Butcher

Hillea Schiller (1)

\section{División Prymnesiophyta}

Clase Prymnesiophyceae Hibberd

Orden Prymnesiales Papenfuss

Familia Phaeocystaceae Lagerheim

Phaeocystis Lagerheim (1) 
Apéndice 2. Continuación.

\author{
Orden Coccosphaerales Haeckel \\ Familia Calciosoleniaceae Kamptner \\ Calciosolenia Gran (1) \\ Familia Coccolithaceae \\ Coccolithus Schwarz (1) \\ cf. Umbilicosphaera Lohmann (1)
}

\section{División Chlorophyta}

Clase Clorophyceae Christensen

Orden Chlorococcales Christensen

Familia Hydrodictyaceae Bourrelly

Pediastrum Meyen (2)

\section{División Euglenophyta}

Clase Euglenophyceae Schoenichen

Orden Euglenales Engler

Familia Euglenaceae Dujardin

Euglena Ehrenberg (1)

Familia Eutreptiaceae Hollande

Eutreptia Perty (1)

\section{División Cyanophyta}

Clase Cyanophyceae Chadefaud

Orden Chroococcales Agnagnostidis et

Komámek
Familia Synechococcales Komámek et Agnagnostidis

Synechococcus Nägeli (1)

Familia Merismopediaceae Elekin Synechocystis Sauvageau (1)

Orden Nostocales Cavalier-Smith

Familia Nostocaceae

Richelia Schmidt in Ostenfeld et Schmidt

(1)

Orden Oscillatoriales Agnagnostidis et

Komámek

Familia Oscillatoriaceae Agnagnostidis et

Komámek

Trichodesmium Ehrenberg (2)

\section{Phylum Zoomastigophora}

Clase Choanoflagellidea

Orden Choanoflagellida

Choanoflagelado (1) 
